Portland State University

PDXScholar

1986

\title{
Elastic-plastic analysis of axisymmetrically loaded isotropic circular and annular plates undergoing large deflections
}

Aiman R. Akileh

Portland State University

Follow this and additional works at: https://pdxscholar.library.pdx.edu/open_access_etds

Part of the Civil Engineering Commons

Let us know how access to this document benefits you.

Recommended Citation

Akileh, Aiman R., "Elastic-plastic analysis of axisymmetrically loaded isotropic circular and annular plates undergoing large deflections" (1986). Dissertations and Theses. Paper 3559.

https://doi.org/10.15760/etd.5441

This Thesis is brought to you for free and open access. It has been accepted for inclusion in Dissertations and Theses by an authorized administrator of PDXScholar. Please contact us if we can make this document more accessible: pdxscholar@pdx.edu. 
AN ABSTRACT OF THE THESIS OF Aiman R. Akileh for the Master of Science in Civil Engineering presented December 19, 1986.

Title: Elastic-Plastic Analysis of Axisymmetrically Loaded Isotropic Circular and Annular Plates Undergoing Large Deflections.

APPROVED BY MEMBERS OF THE THESIS COMMITTEE:
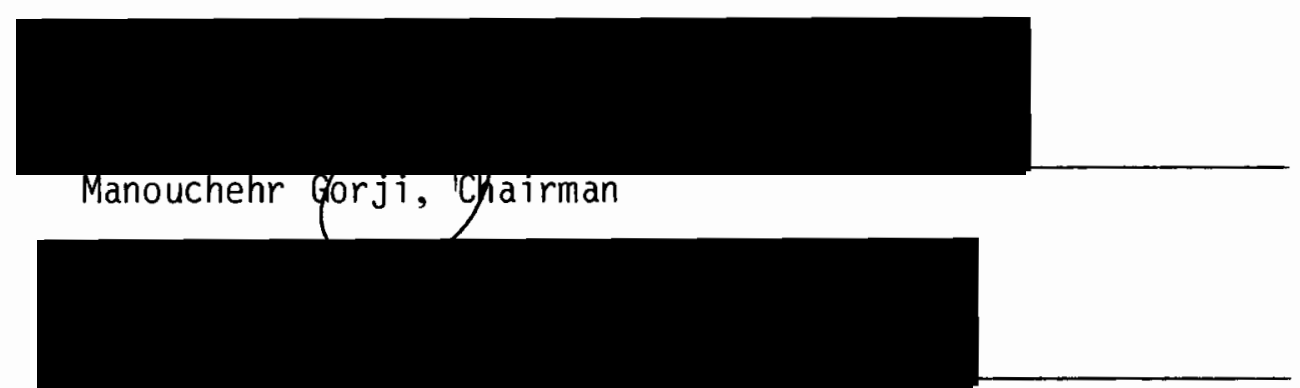

Wenderin H. Mueller

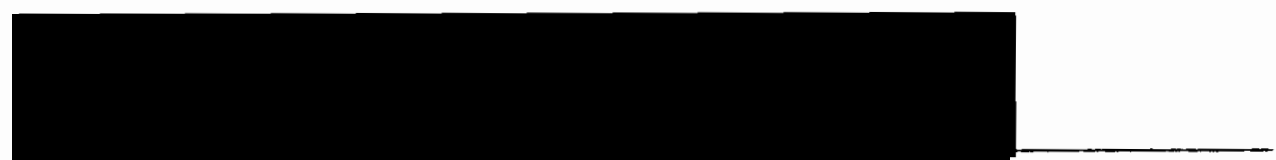

Herman J. Migliorel

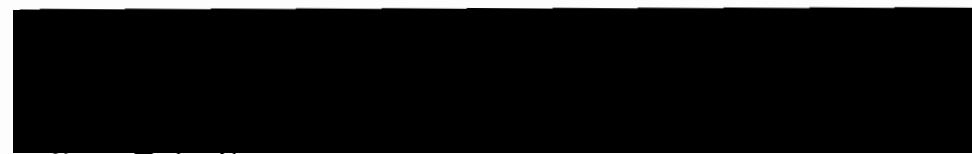

Nan-leh Hsu

The concept of load analogy is used in the elastic and elastic-plastic analysis of isotropic circular and annular plates undergoing moderately large deflection. The effects of the nonlinear terms of lateral displacement and the plastic strains are considered as additional fictitious lateral loads, edge moments, and in-plane forces acting on the plate. The solution of an elastic or elastic-plastic Von 
Karman type plate is hence reduced to a set of two equivalent elastic plate problems with small displacements, namely, a plane problem in elasticity and a linear elastic plate bending problem. The method of finite element is employed to solve the plane stress problem. The large deflection solutions are then obtained by utilizing the solutions of the linear bending problems through an iterative numerical scheme. The flow theory of plasticity incorporating a Von Mises layer yield criterion and the Prandtl-Reuss associated flow rule for strain hardening materials is employed in this approach.

Nonlinear elastic results for simply supported and clamped circular and annular plates are found to be in excellent agreement with the available solutions. The nonlinear elastic-plastic results show that the lateral displacement is increased by the plastic strain while the maximum fiber stresses are considerably relieved. Furthermore, the method of solution used in this study is found to be computationally efficient and offers an alternative method for obtaining nonlinear solutions to circular and annular plate bending problems. The present formulation can be easily extended to include other sources of nonlinearity such $a_{n}^{\zeta}$ creep and relaxation in the analysis. Moreover, the approach is also well suited for the nonlinear analysis of circular and annular composite plates. 


\title{
ELASTIC-PLASTIC ANALYSIS OF AXISYMMETRICALLY \\ LOADED ISOTROPIC CIRCULAR AND ANNULAR \\ PLATES UNDERGOING LARGE DEFLECTIONS
}

by

AIMAN RAJI AKILEH

A thesis submitted in partial fulfillment of the requirements for the degree of

\author{
MASTER OF SCIENCE \\ IN \\ CIVIL ENGINEERING
}

Portland State University 
TO THE OFFICE OF GRADUATE STUDIES AND RESEARCH:

The members of the Committee approve the thesis of Aiman R. Akileh presented December 19, 1986.

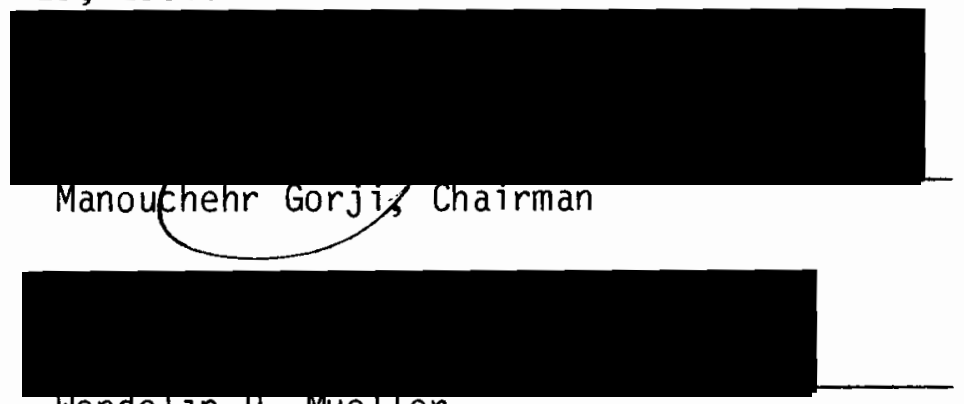

Wendel in H. Mueller

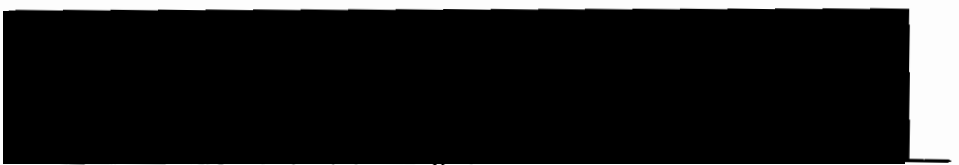

Herman J. MiglioreV

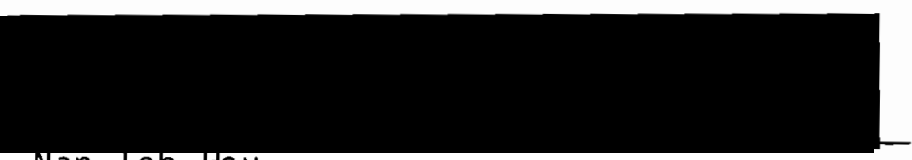

Nan-len Hsu
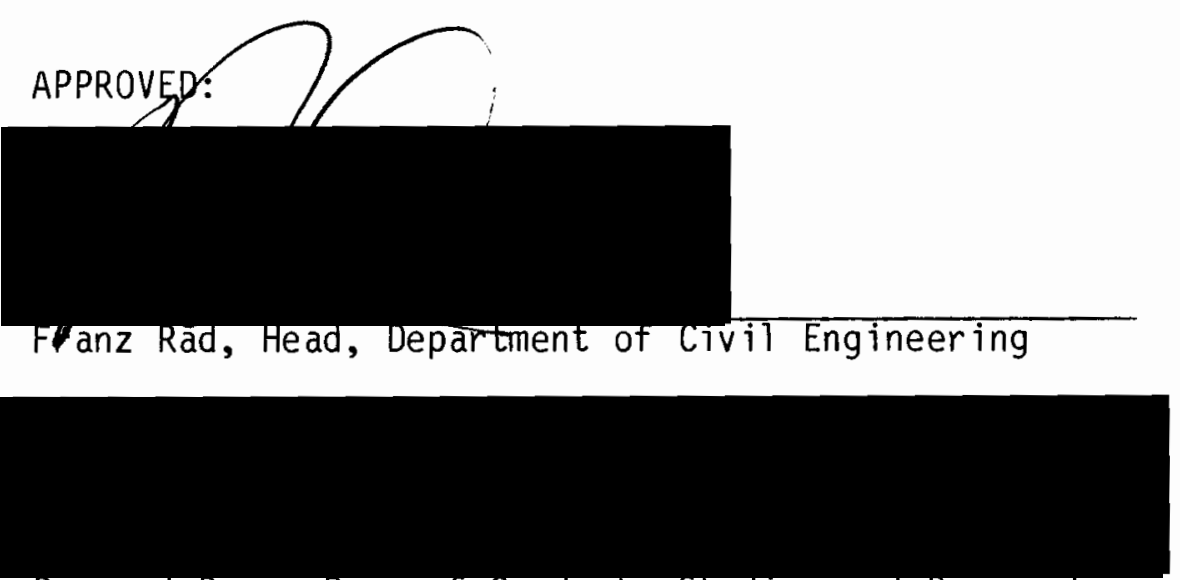

Bernard Ross, Dean of Graduate Studies and Research 
Dedicated to my parents

\author{
Raji S. Akileh
}

Amina Darwish

.... In partial payment of a debt

I shall always owe .... 


\section{ACKNOWLEDGEMENTS}

This investigation was carried out under the supervision of Dr. Manouchehr Gorji. I an deeply indebted to Dr. Gorji for the countless hours, tireless dedication, patient guidance, valuable suggestions and the encouragement rendered throughout the course of this study. I also wish to convey my warmest thanks to the other members of the thesis committee: Dr. Nan-Teh Hsu, Dr. Herman J. Migliore, and Dr. Wendelin H. Mueller for their helpful comments and suggestions.

I would like to express my grateful appreciation to the School of Engineering and Applied Science and to the Head of the Department of Civil Engineering, Dr. Franz Rad, for his help, guidance, and leadership.

Thanks are also due to Mr. Jon Griffin and to Portland State University's computing center for their computational support. The data entry support of Miss Tina Lemon, Mr. Ammar Barghouti, and my brother Riad Akileh is gratefully acknowledged.

I would like to extend my deepest graditude to the members of my family for all the sacrifices and the generous financial and emotional support. Much appreciation is also due to all of my friends, too many to mention individually, for their love and support.

I am especially indebted to Mr. and Mrs. William D. Mills and to the people of Oregon for making this beautiful state a sweet home away from 
home .

I would like to thank Ms. Julie Schemmel for the excellent typing of this thesis.

I thank God for the ability to learn and the ambition to endure the challenges of acquiring an education.

Finally, I am forever indebted to my dear and loving friend Joy Shcutte for her understanding, encouragement and patience throughout my undergraduate and graduate work. 
TABLE OF CONTENTS

Page

ACKNOWLEDGEMENTS .................... iv

LIST OF FIGURES .....................................

CHAPTER

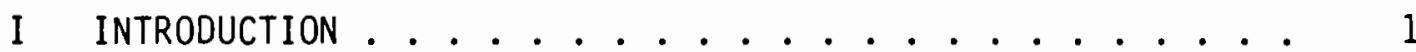

Survey of Previous Work . . . . . . . . . . 1

Statement of Objectives .......... 8

II MATHEMATICAL FORMULATION . . . . . . . . . 10

Assumptions ............... 10

Constitutive Relations ........... . 11

Governing Equilibrium Equations ......... 21

III PLASTIC STRESS-STRAIN RELATIONS . . . . . . . . 27

Von Mises Initial Yield Criterion . . . . . . . 30

Subsequent Yield Surfaces ............. 31

Prandt1-Reuss Incremental Plastic Stress-Strain Relations ............. 35

IV METHOD OF SOLUTION . . . . . . . . . . 40

Equivalent Load Theory . . . . . . . . . . 40

Incremental Numerical Scheme ......... . 45

Numerical Solution Convergence, Accuracy and

Stability............... 
$\checkmark$ NUMERICAL RESULTS AND DISCUSSION . . . . . . . . . . 50

Large Deflection, Elastic Results . . . . . . . 53

Simply Supported Circular Plate

Clamped Circular Plate

Simply Supported Annular Plate

Clamped Annular Plate

Large Deflection, Elastic-Plastic Results . . . . .

Simply Supported Circular Plate

Clamped Circular Plate

Simply Supported Annular Plate

Clamped Annular Plate

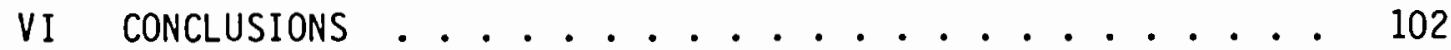
BIBLIOGRAPHY . . . . . . . . . . . . . . . . . . 104

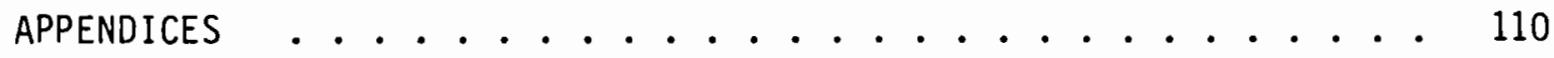

A Notation.............................. 111

B Analytical Solutions of Linear Plate Bending Probems . . 114

Simply Supported Circular Plate . . . . . . . 114

Clamped Circular Plate ........... . 115

Simply Supported Annular Plate . . . . . . . . 117

Clamped Annular Plate ........... 125 


\section{LIST OF FIGURES}

F IGURE

Page

1 Coordinate system at the middle surface and sign

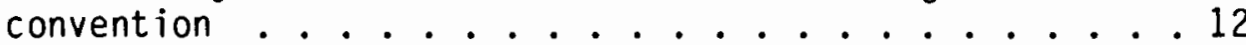

2 Circular and annular plate notation ......... 13

3 Radial displacement of point C . . . . . . . 15

4 Equilibrium of a circular plate ...........22

5 Graphical representation of the Von Mises initial yield criterion for axisymmetricaliy loaded plates . . . 32

6 Actual and corresponding equivalent plate boundaries . . . 44

7 Deflection at the center of a uniformly loaded simply supported circular plate $(v=0.25) \ldots 58$

8 Variation of radial stresses with load for a uniformly loaded simply supported circular plate $(v=0.25) . .59$

9 Deflection at the center of a uniformly loaded clamped circular plate $(v=0.3) \ldots 60$

10 Variation of radial stresses with deflection for a uniformly loaded clamped circular plate $(\nu=0.3) \ldots 61$

11 Deflection at the inner edge of a uniformly loaded simply supported annular plate $(\nu=0.3, b / a=0.25) .62$

12 Variation of bending stresses with load for a uniformly loaded simply supported annular plate $(v=0.3, b / a=0.25) \ldots 63$

13 Variation of membrane stresses with load for a uniformly loaded simply supported annular plate $(v=0.3, b / a=0.25) \ldots \ldots 64$

14 Deflection at the inner edge of a uniformly loaded clamped annular plate $(\nu=0.3, b / a=0.25) \ldots 65$ 
15 Variation of bending stresses with load for a uniformly loaded clamped annular plate $(v=0.3, b / a=0.25) . .66$

16 Variation of membrane stresses with load for a uniformly loaded clamped annular plate $(\nu=0.3, b / a=0.25) . .67$

17 Deflection at the center of a uniformly loaded simply supported circular plate $(\nu=0.3)$........ 68

18 Variation of membrane stresses with load for a uniformly loaded simply supported circular plate $(\nu=0.3)$. . . 69

19 Variation of total stresses with load for a uniformly loaded simply supported circular plate $(\nu=0.3) . . .70$

20 Distribution of effective plastic strain for a uniformly loaded simply supported circular plate $(\nu=0.3$, $\left.q(a / h)^{4} / E=7.15\right)$................ 71

21 Deflection profile for a uniformly loaded simply supported circular plate $(v=0.3$, $\left.q(a / h)^{4} / E=7.15\right)$

22 Radial stress profile for a uniformiy loaded simply supported circular plate $(v=0.3$, $\left.q(a / h)^{4} / E=7.15\right)$

23 Tangential stress profile for a uniformly loaded simply supported circular plate $(\nu=0.3$, $\left.q(a / h)^{4} / E=7.15\right)$

24 Sectional moment profile for a uniformly loaded simply supported circular plate $(\nu=0.3$, $\left.q(a / h)^{4} / E=7.15\right)$

25 Deflection at the center of a uniformly loaded clamped circular plate $(v=0.3)$. . . . . . . . . . 76

26 Variation of membrane stresses with load for a uniformly loaded clamped circular plate $(\nu=0.3)$. . . . . . 77

27 Variation of total stresses with load for a uniformly loaded clamped circular plate $(\nu=0.3)$. . . . . . 78

28 Distribution of effective plastic strain for a uniformly loaded clamped circular plate $(\nu=0.3$, $\left.q(a / h)^{4} / E=5.96\right)$. . . . . . . . . . . . . 79

29 Deflection profile for a uniformly loaded clamped circular plate $(\nu=0.3$, $q(a / h) 4 / E=5.96)$ 
30 Radial stress profile for a uniformly loaded clamped circular plate $(\nu=0.3$, $\left.q(a / h)^{4} / E=5.96\right)$

31 Tangential stress profile for a uniformly loaded clamped circular plate $(\nu=0.3$, $\left.q(a / h)^{4} / E=5.96\right)$

32 Sectional moment profile for a uniformly loaded clamped circular plate $(v=0.3$, $\left.q(a / h)^{4} / E=5.96\right)$

33 Deflection at the inner edge of a uniformly loaded simply supported annular plate $(v=0.3, \mathrm{~b} / \mathrm{a}=0.25) . . . .84$

34 Variation of membrane stresses with load at the inner edge of a uniformly loaded simply supported annular plate $(v=0.3, b / a=0.25) . . . . . . . . . .85$

35 Variation of membrane stresses with load at the outer edge of a uniformly loaded simply supported annular plate $(\nu=0.3, b / a=0.25)$

36 Variation of total stresses with load for a uniformly loaded simply supported annular plate $(\nu=0.3$, $\mathrm{b} / \mathrm{a}=0.25$ )

37 Distribution of effective plastic strain for a uniformly loaded simply supported annular plate $(v=0.3$, $\left.\mathrm{b} / \mathrm{a}=0.25, \mathrm{q}(\mathrm{a} / \mathrm{h})^{4} / E=3.58\right)$

38 Deflection profile for a uniformly loaded simply supported annular plate $(v=0.3, b / a=0.25$, $\left.q(a / h)^{4} / E=3.58\right)$

39 Radial stress profile for a uniformly loaded simply supported annular plate $(v=0.3, b / a=0.25$, $\left.q(a / h)^{4 / E}=3.58\right) \quad$................. 90

40 Tangential stress profile for a uniformly loaded simply supported annular plate $(\nu=0.3, b / a=0.25$, $\left.q(a / h)^{4} / E=3.58\right)$

41 Sectional moment profile for a uniformly loaded simply supported annular plate $(\nu=0.3, b / a=0.25$, $\left.q(a / h)^{4} / E=3.58\right)$

42 Deflection at the inner edge of a uniformly loaded clamped annular plate $(\nu=0.3, b / a=0.25) . . . . . . .93$ 
43 Variation of membrane stresses with load at the inner edge of a uniformly loaded clamped annular plate $(v=0.3$,

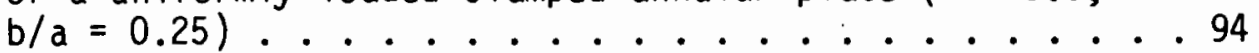

44 Variation of membrane stresses with load at the outer edge of a uniformly loaded clamped annular plate $(v=0.3$,

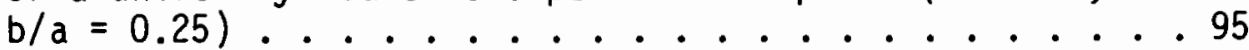

45 Variation of total stresses with load for a uniformiy loaded clamped annular plate $(v=0.3, \mathrm{~b} / \mathrm{a}=0.25) . .96$

46 Distribution of effective plastic strain for a uniformly loaded clamped annular plate $(\nu=0.3, b / a=0.25$, $\left.q(a / h)^{4} / E=5.96\right)$................. 97

47 Deflection profile for a uniformly loaded clamped annular plate $\left(\nu=0.3, b / a=0.25, q(a / h)^{4} / E=5.96\right) . . . .98$

48 Radial stress profile for a uniformly loaded clamped annular plate $\left(v=0.3, b / a=0.25, q(a / h)^{4} / E=5.96\right) .99$

49 Tangential stress profile for a uniformly loaded clamped annular plate $\left(v=0.3, b / a=0.25, q(a / h)^{4} / E=5.96\right) . .100$

50 Sectional moment profile for a uniformly loaded clamped annular plate $\left(\nu=0.3, b / a=0.25, q(a / h)^{4} / E=5.96\right) . .101$

51 Decomposition of two linear annular plate bending problems 
CHAPTER I

INTRODUCTION

When the lateral displacement of a plate is not small in comparison with its thickness, the membrane forces play a more dominant role in carrying the load, and hence the second order effects of lateral displacement on the membrane stresses need to be considered $(1,2,3)$. In cases in which the vertical deflections are no longer small in comparison with the thickness of the plate but are still small as compared with the other dimensions, the analys is of the problem must be extended to include the strain of the middle plane of the plate.

Nonlinear differential equations considering these effects were first given by Von Karman (4). Solutions based on these nonlinear differential equations are known as large deflection solutions in the sense of Von Karman Theory. These differential equations have been used in the elastic analys is of isotropic and orthotropic circular and annular plates with various edge conditions subject to uniform lateral pressure, axisymmetric line loads, or concentrated loads applied at the center of the plate.

\section{SURVEY OF PREVIOUS WORK}

Various investigators have provided solutions for such nonlinear elastic problems. Among the earlier contributions is the work of Way (5) 
which presents a series solution for uniformly loaded clamped circular plates. Other series solutions for calculating finite deflections of elastic simply supported and clamped circular plates have been proposed by Alwar, et al. $(6,7)$, Berger (8), Federhofer and Egger (9), Mansfield (10), Stoker (11), and Timoshenko and Woinowsky-Krieger (3). On the other hand, various investigations of nonlinear elastic circular plates have been attempted using numerical methods such as finite differences $(12,13)$, finite element (14), dynamic relaxation (15, 16), perturbation (17-22), integral collocation method (23) and rateform linearization (24). Recently, Dumir, et al. (25-27) used point collocation method together with Newmark- $B$ scheme to analyze the static and transient nonlinear response of isotropic and orthotropic circular plates.

A number of solutions for elastic annular plates with various inner and outer edge conditions have also been obtained by different researchers. Wempner and Schmidt (28) presented a series solution for an isotropic annular plate with simply supported movable outer edge subjected to a uniform line load at the free inner edge. Yeh (29) has given an approximate solution using the perturbation method for a $\mathrm{clamped}$ imnovable isotropic annular plate with a plugged hole under a concentrated load at the plug. Mah (13) presented a finite difference solution for isotropic annular plates with simply supported and clamped immovable edges under a uniform load. Recently, Dumir, et al. (30) presented a large deflection analysis of orthotropic annular plates under uniformly distributed loads using the method of interior global orthogonal point collocation. Alwar and Reddy (7) analyzed the static and dynamic response of isotropic and 
orthotropic annular plates using a Chebyshev series expansion, while Turvey (16) considered the behavior of tapered annular isotropic plates under a uniformly distributed load using the dynamic relaxation technique.

All of the aforementioned methods were based on Von Karman's nonlinear differential equations. The accuracy of these methods in yielding numerical results is dependent on the number of terms utilized in the series solution or the number of grid points used in the case of numerical schemes. Furthermore, the foregoing solution methods assume elastic behavior and, hence, are not applicable to plates loaded beyond the elastic limit.

Small deflection elastic-plastic solutions for axisymmetrically loaded circular and annular plates have been given by many investigators. Sokolovsky $(31,32)$ presented solutions for simply supported plates employing Henky type deformation theory of plasticity together with Von Mises yield condition. Three loading conditions were investigated: a uniformly distributed load, a uniformly distributed load over an inner circular portion of the plate, and a concentrated load at the center of the plate. Several other investigators extended Sokolovsky's solution to plates with variable thickness (33), other loading conditions (34), and to plates with clamped boundaries (35). Limit analysis has also been applied extensively to obtain the collapse loads of isotropic circular plates employing various yield criterion, loadings, and support conditions (36-38). An early attempt to estimate the deflections of elasto-plastic circular plates using incremental analysis based on the flow theory of plasticity was carried out by Haythornthwaite (39). The yield condition of 
Tresca and the associated flow rule were employed. The key assumption was made that at any point within the plate the entire thickness was either fully elastic or fully plastic. An example of an annular plate was given. Lackman (40) employed an analogy between plastic strains and transverse loads originally advanced by Lin (41) to obtain solutions for a uniformly loaded, simply supported circular plate made of strain hardening 2024-T4 aluminum. The plate was divided into 40 layers along its thickness. Unlike Haythornthwaite's full section plasticity model, the plastic strains were considered to vary in the thickness direction. The incremental plastic strains were obtained by the use of the Reuss-Mises incremental polyaxial inelastic stress-strain relations. Popov, et al. (42) treated the elastic-plastic bending of simply supported and clamped circular plates by employing Von Mises-layer yield criterion and Prandtl-Reuss incremental plastic stress-strain relations. The material was assumed to be elastic-perfectly plastic.

Very few large deflection elasto-plastic solutions of circular and annular plates have been reported and even fewer analytical-experimental correlations exist (43). Naghdi (44) was the first to undertake full-range analysis of axisymmetrically loaded circular plates. His numerical scheme employed plastic stress-strain relations based on the deformation theory of plasticity. The validity of his approach was established by comparing deflections for a simply supported circular plate loaded at the center with those obtained from experiment. Tensile stress-strain and octahedral shear stress-shear strain curves for 24S-T aluminum were also provided. Ohashi, et al. (35, 45-48) obtained solutions for elastic-perfectly plastic 
circular and annular plates using an analytical technique based on the deformation theory of plasticity. Their technique presents an extension of Sokolovsky's small deflection analysis of the same problem. Transversely loaded circular plates with simply supported (45) and clamped $(46,35)$ immovable edges along with annular plates (47) with free inner, simply supported immovable outer edges were tested. The validity of their analytical solutions was established based on favorable comparison with the experimental results. Myszkowski (49) developed a simplified version of the elasto-plastic constitutive equations used by Ohashi, et al. and incorporated them into a "shooting-type" analys is to solve circular plate problems with simply supported and clamped edges under transverse pressure. Recently, Turvey $(50,51)$ incorporated Myszkowski's constitutive relations into the dynamic relaxation method to obtain large elasto-plastic solutions to uniformly loaded circular plates. Several sets of results for simply supported and clamped plates made of either mild or high yield steel with constant and variable thicknesses were presented. The accuracy of this approach was verified through favorable comparison with experimental results obtained by Myszkowski (49) and Ohashi and Murakami (39).

An interesting simplified and design oriented full-range analysis of transversely loaded simply supported circular plates was published by Sherbourne, et al. (52). In their analysis a cross section of the plate was assumed to consist of two different types of elements, flexure and membrane elements, which were assumed to act independently without any interaction except in bond. Initially, the transverse pressure was assumed 
to be supported solely by flexure action. When the flexure capacity of the plate section was exhausted, membrane action was assumed to take up the additional load-carrying requirement. Although Sherbourne, et al. attempted to establish the validity of their analysis by comparing lateral deflections of the solutions with simply supported plate test data, their methodology was regarded as too simplistic for general application (43).

The principal weakness of the approximate full-range analyses outlined above is that they are incapable of taking proper account of any local elastic unloading which may arise as the lateral pressure increases and membrane action develops in the plate. These analyses, being based on the deformation theory of plasticity, are load-path independent. In reality, however, the plastic strain state is load-path dependent $(2,43$, 53-55). The incremental analyses based on the flow theory of plasticity permit the load-path to be taken into proper account and, therefore, are regarded as being more accurate representations of the physical situation. Only a few investigations of elastic-plastic circular plates undergoing large deflections based on the incremental flow theory of plasticity have been reported in the open literature. A brief account of these studies is given below:

In 1969, Crose and Ang (56) proposed a discrete model of a plate consisting of a system of flexible nodes, rigid bars, and torsional springs to analyze the large deflection elastic-plastic behavior of circular plates. Von Mises yield criterion was employed. The incremental plastic strains were obtained by using the Prandtl-Reuss flow rule for elastic-perfectly plastic materials. The reliability of the model was 
verified through favorable comparison with theoretical (3) and experimental (57) results. Tanaka (58) presented an iterative scheme for solving the finite-difference approximations to the governing system of equations. His analys is employed Von Mises-1ayer yield criterion and allowed all linear combinations of isotropic and kinematic strain hardening to be accounted for. This analysis was subsequently used by Hamada, et al. (59) to explore the response of circular plates under slowly varying cyclic loading. Recentiy, Turvey and $L$ im (43) employed the dynamic relaxation method to obtain solutions for the finite-difference approximations to the governing equations of elasto-plastic circular plates undergoing large deflections. Their analysis was based on the flow theory of plasticity and incorporated a Von Mises-layer plasticity model, an Ilyushin and Ivanov full-section plasticity model, and the Prandt]-Reuss associated flow rule for elastic-perfectly plastic materials. It was observed that the layer analysis predicts the measured deflections more accurately than the full-section analysis. The analytical-experimental deflection correlation appeared to be accurate for slender $c l$ amped $p l a t e s$ and less accurate for thick plates. This finding is not surprising since the effect of transverse shear deformation was not accounted for. However, the analytical-experimental correlation for simply supported plates appeared to be less favorable.

In all the foregoing studies the plate is assumed to behave in an elastic-perfectly plastic manner. Therefore, the resulting solutions can only be expected to yield approximate results for plates exhibiting strain-hardening characteristics. Moreover, the solution techniques are 
applicable to circular plates without a hole at the center and cannot easily be extended to annular plates. Additionally, the need for sophisticated modern full-range analytical techniques, i.e., techniques which take proper account of the interaction between large deflections (in the Von Karman sense) and elasto-plastic material characteristics, is clearly evident. These techniques must be simple, amenable to programming, and highly efficient for the practicing engineer to consider, yet they must be accurate and highly versatile to be regarded worthy of general application. Furthermore, full-range investigations based on the incremental flow theory of plasticity incorporating strain-hardening materials for circular and annular plates are clearly lacking.

This study attempts to present a simple, yet rather versatile numerical technique for the analysis of large deflection elastic and elasto-plastic circular and annular plates. An attempt to fill the void regarding the use of incremental flow theory of plasticity incorporating strain-hardening materials in full-range analyses will be initiated.

\section{STATEMENT OF OBJECTIVES}

The first objective of this study is to assess the accuracy and efficiency of the equivalent load concept $(1,2,40,41,60-63)$ in the analysis of axisymmetrically loaded isotropic circular and annular elastic plates undergoing large deflections. The motivation for this step has been to validate the results of nonlinear elastic problems. The second objective of this study is to extend the equivalent load concept to the large deflection elastic-plastic analysis of axisymmetrically loaded 
isotropic circular and annular plates. This analysis is based on the incremental flow theory of plasticity and incorporates a Von Mises-layer yield criterion and the Prandtl-Reuss associated flow rule for strain-hardening materials.

Accordingly, the equivalent load concept is employed in the large deflection (in the Von Karman sense) analysis of elastic and elasto-plastic circular and annular isotropic plates. The nonlinear terms of lateral displacement and the plastic strain gradients are considered as an additional set of body and surface forces acting on the plate. The solution of an elastic or elasto-plastic Von Karman type plate is hence reduced to a set of two equivalent elastic plate problems undergoing small displacements, namely, a plane problem in elasticity and a linear elastic plate bending problem. The numerical method of finite element (64) is employed to solve the plane stress problem. The large deflection solutions are then obtained by employing the solutions of the linear plate bending problems ( 3,65 , Appendix B) through an iterative numerical scheme. 
CHAPTER II

MATHEMATICAL FORMULATION

The constitutive relations and governing equilibrium equations derived herein describe the axisymmetric large deflection behavior of thin circular and annular isotropic plates loaded beyond the elastic limit by uniformly distributed transverse loads. In the development of these relations the following assumptions are made:

1. The material is homogeneous and isotropic.

2. The body forces are negligible in comparison with the applied loads.

3. The lateral displacement of the plate is large (in the Von Karman sense). Hence, the second order effects of lateral displacement must be considered.

4. The curvature of the $p l a t e$ may be approximated by $\left(-\frac{d^{2} w}{d r^{2}}\right.$

5. The Kirchhoff's assumption is valid, i.e., the normal to the middle surface of the plate remains straight, unextended, and normal to the middle surface after deformation. Hence, transverse shear strains are neglected.

6. The stress normal to the midplane is small compared with other stress 
components and may, therefore, be neglected.

Consequently, the resulting governing equations are in the realm of classical large deflection theory.

\section{CONSTITUTIVE RELATIONS}

Let $r, t, z$ be a curvilinear coordinate system with the $r t p l a n e$ coinciding with the middle surface of the plate in its unloaded initial state and $z$ axis normal to it and pointing downward. Let $u_{0}$ and $w$ be the components of displacement of the middle surface in the radial and lateral directions, respectively. The coordinate system and the sign convention for forces are shown in Fig. 1.

Consider a circular or annular plate of uniform thickness $h$ under the action of a uniform lateral load $q$ as shown in Fig. 2 . Since the plate and loading are axisymmetric, the displacements, stresses, and strains in the plate are aiso axisymmetric, and hence

$$
\begin{aligned}
& \frac{d}{d t}=0 \\
& \sigma_{r t}=\varepsilon_{r t}=0
\end{aligned}
$$

where $\sigma$ and $\varepsilon$ denote the stress and strain components, respectively. The slope $\phi$ of the deformed middle surface in the $r z p l a n e$ is given by

$$
\phi=-w, r
$$




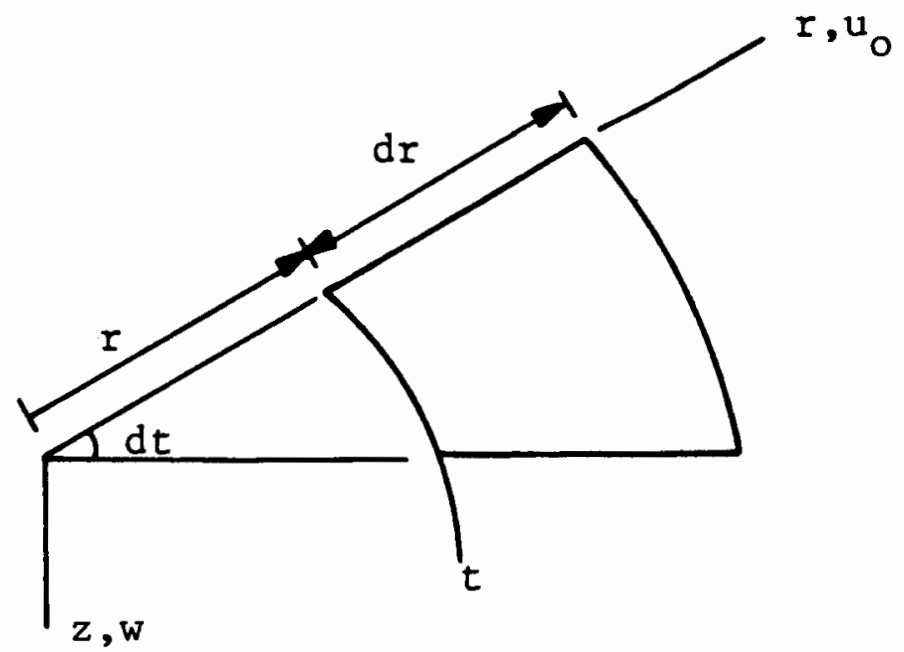

(a) COORDINATE SYSTEM

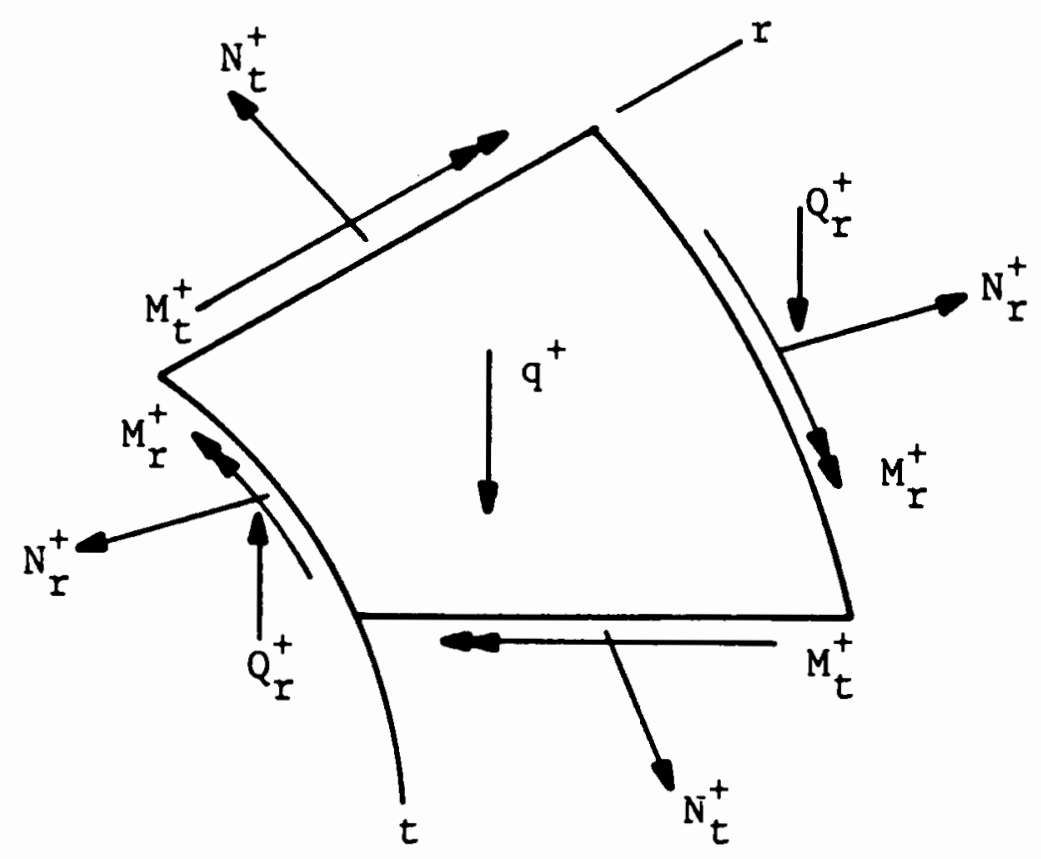

(b) SIGN CONVENTION

FIGURE 1. COORDINATE SYSTEM AT MIDDLE SURFACE AND SIGN CONVENTION 


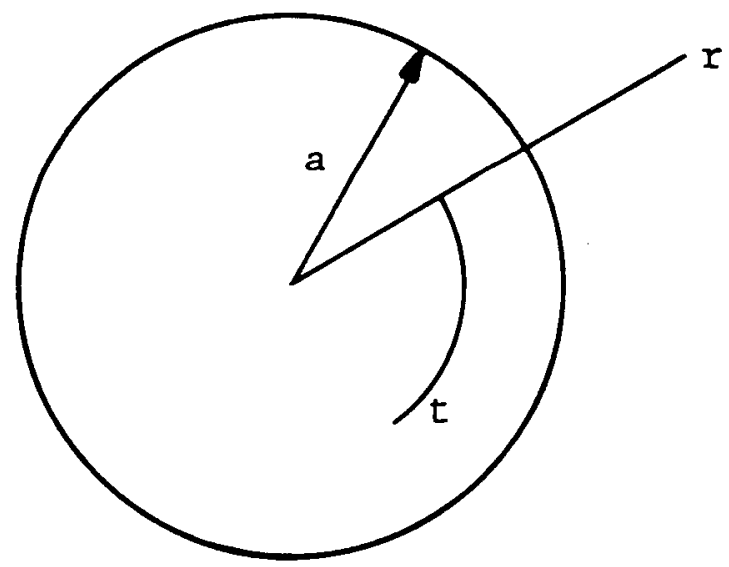

(a) CIRCULAR PLATE (TOP VIEW)

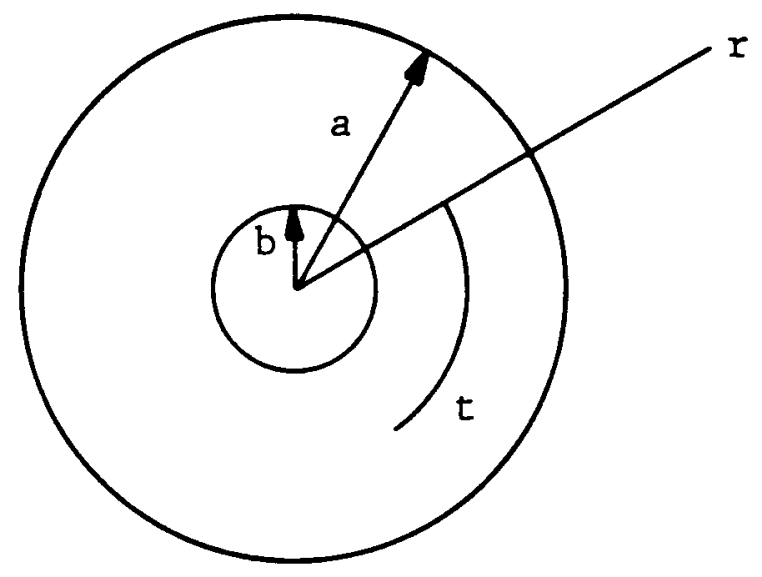

(b) ANNULAR PLATE (TOP VIEW)

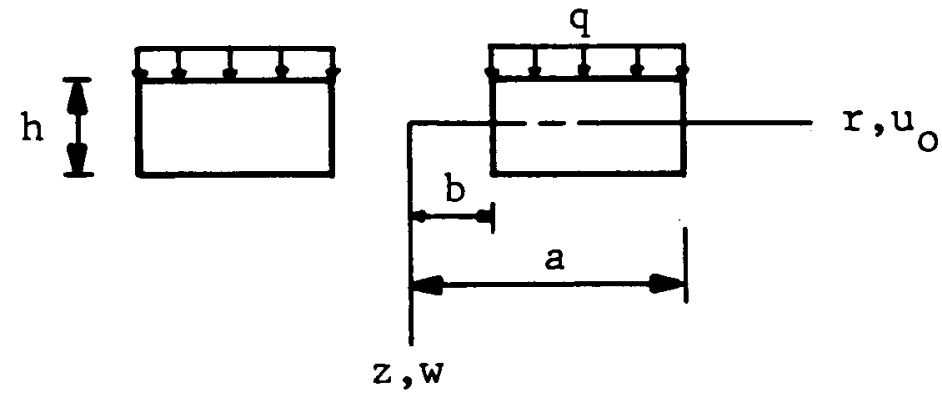

(c) ANNULAR PLATE (FRONT VIEW)

FIGURE 2. CIRCULAR AND ANNULAR PLATE NOTATION 
where comma denotes differentiation. The curvature of the plate may be approximated by

$$
k=\phi, r=-w, r r
$$

Utilizing the stated assumptions, the radial displacement field for an axisymetrically loaded plate may be expressed as

$$
u(r, z)=u_{0}(r)-z w, r
$$

in which $u_{0}(r)$ denotes the radial displacement of the middle surface and $w$ denotes the lateral deflection as shown in Fig. 3 . The nonlinear strain-displacement relations for a Von Karman type plate are given by (3, 65)

$$
\begin{aligned}
& \varepsilon_{r}=u_{r}+\frac{1}{2} w_{r}^{2}=u_{0, r}+\frac{1}{2} w_{r}^{2}-z w, r r \\
& \varepsilon_{t}=\frac{u}{r}=\frac{u_{0}}{r}-\frac{z}{r} w, r
\end{aligned}
$$

where $w^{2}, r$ represents the second order effect of lateral displacement.

For a plate loaded beyond the elastic limit, the total component of strain may be expressed as the sum of its elastic and plastic components:

$$
\varepsilon_{r}=\varepsilon_{r}^{e}+\varepsilon_{r}^{\prime \prime}
$$




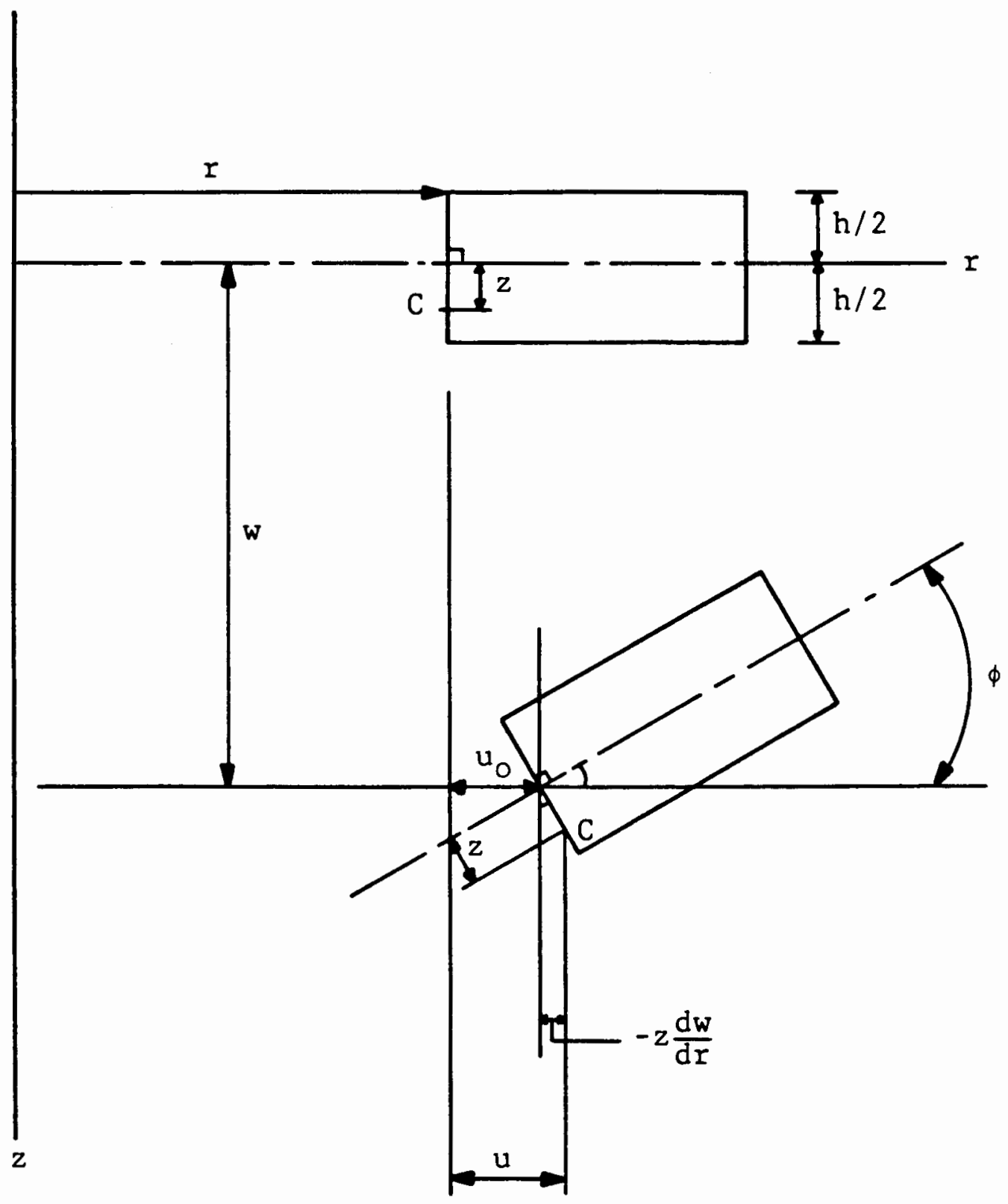

FIGURE 3. RADIAL DISPLACEMENT OF POINT C 


$$
\varepsilon_{t}=\varepsilon_{t}^{e}+\varepsilon_{t}^{\prime \prime}
$$

The stress-strain relations for a plane stress problem are given by

$$
\begin{aligned}
& \sigma_{r}=\frac{E}{1-v^{2}}\left(\varepsilon_{r}^{e}+v \varepsilon_{t}^{e}\right)=\frac{E}{1-v^{2}}\left[\varepsilon_{r}+v \varepsilon_{t}-\left(\varepsilon_{r}^{\prime \prime}+v \varepsilon_{t}^{\prime \prime}\right)\right] \\
& \left.\sigma_{t}=\frac{E}{1-v^{2}}\left(\varepsilon_{t}^{e}+v \varepsilon_{r}^{e}\right)=\frac{E}{1-v^{2}} \varepsilon_{t}+v \varepsilon_{r}-\left(\varepsilon_{t}^{\prime \prime}+v \varepsilon_{r}^{\prime \prime}\right)\right]
\end{aligned}
$$

in which $E$ and $\nu$ are Young's modulus and Poisson's ratio, respectively. The nonlinear stress-displacement relations are obtained by substituting relations (2.5) into relations $(2.7)$. On this basis, we may write

$$
\begin{aligned}
& \sigma_{r}=\frac{E}{1-v^{2}}\left[u_{0, r}+\frac{\nu}{r} u_{0}-z\left(w, w_{r r}+\frac{v}{r} w_{r}\right)+\frac{1}{2} w_{r}^{2}-\left(\varepsilon_{r}^{\prime \prime}+v \varepsilon_{t}^{\prime \prime}\right)\right] \\
& \sigma_{t}=\frac{E}{1-v^{2}}\left[\frac{u_{0}}{r}+v u_{0, r}-z\left(v w_{r r}+\frac{1}{r} w_{r}\right)+\frac{\nu}{2} w_{r}^{2}-\left(v \varepsilon_{r}^{\prime \prime}+\varepsilon_{t}^{\prime \prime}\right)\right]
\end{aligned}
$$

The sectional forces and moments are obtained by integrating the stress components across the thickness of the plate. These relations are defined by

$$
\begin{aligned}
& N_{r}=\int \sigma_{r} d z \\
& N_{t}=\int \sigma_{t} d z
\end{aligned}
$$




$$
\begin{aligned}
& M_{r}=\int \sigma_{r} z d z \\
& M_{t}=\int \sigma_{t} z d z
\end{aligned}
$$

where sectional forces and moments are expressed per unit length of a given cross section of the plate. Substituting relations (2.8) into relations (2.9) and carrying out the required differentiations yield

$$
\begin{aligned}
& N_{r}=N_{r}^{E}+\bar{N}_{r}-N_{r}^{\prime \prime} \\
& N_{t}=N_{t}^{E}+\bar{N}_{t}-N_{t}^{\prime \prime} \\
& M_{r}=M_{r}^{E}-M_{r}^{\prime \prime} \\
& M_{t}=M_{t}^{E}-M_{t}^{\prime \prime}
\end{aligned}
$$

where

$$
\begin{aligned}
& N_{r}^{E}=\frac{E h}{1-v^{2}}\left(u_{0, r}+\frac{v}{r} u_{0}\right) \\
& N_{t}^{E}=\frac{E h}{1-v^{2}}\left(\frac{u_{0}}{r}+v u_{0, r}\right) \\
& M_{r}^{E}=-D\left(w, w_{r}+\frac{v}{r} w, r\right)
\end{aligned}
$$




$$
\begin{aligned}
& M_{t}^{E}=-D\left(\frac{1}{r} w,_{r}+v w_{r}\right) \\
& D=\frac{E h^{3}}{12\left(1-v^{2}\right)} \\
& \bar{N}_{r}=\frac{E h}{2\left(1-v^{2}\right)} w^{2}{ }_{r} \\
& \bar{N}_{t}=\frac{E h v}{2\left(1-v^{2}\right)} w^{2}{ }^{2} \\
& N_{r}^{\prime \prime}=\frac{E}{1-v^{2}} \int\left(\varepsilon_{r}^{\prime \prime}+v \varepsilon_{t}^{\prime \prime}\right) d z \\
& N_{t}^{\prime \prime}=\frac{E}{1-v^{2}} \int\left(\varepsilon_{t}^{\prime \prime}+v \varepsilon_{r}^{\prime \prime}\right) d z \\
& M_{r}^{\prime \prime}=\frac{E}{1-v^{2}} \int\left(\varepsilon_{r}^{\prime \prime}+v \varepsilon_{t}^{\prime \prime}\right) z d z \\
& N^{2}
\end{aligned}
$$

Expressions (2.11) represent the sectional forces and moments of an axisymmetrically loaded elastic plate with small deflections $(3,65)$, whereas the second order effect of lateral displacement and the influence of the inelastic strains on the forces and moments are given by expressions (2.13) and (2.14), respectively. 
The shear force $Q_{r}$, shown in more detail in the next section, is obtained by considering the equilibrium of an element of the plate and is given for convenience as

$$
Q_{r}=M_{r, r}+\frac{M_{r}-M_{t}}{r}
$$

Substituting relations (2.10.c \& d) and (2.11.c \& d) into equation (2.15) yields

$$
Q_{r}=Q_{r}^{E}-Q_{r}
$$

where

$$
\begin{aligned}
& Q_{r}^{E}=-D\left(w_{r r r}+\frac{1}{r} w_{r r}-\frac{1}{r^{2}} w_{r}\right) \\
& Q_{r}^{\prime \prime}=M_{r, r}^{\prime \prime}+\frac{M_{r}^{\prime \prime}-M_{t}^{\prime \prime}}{r}
\end{aligned}
$$

Again, we note that expression (2.17.a) represents the shear force of an axisymmetrically loaded elastic plate undergoing small deflections (3, $65)$ in contrast to expression (2.17.b) which refers to the inelastic shear force.

Substituting relations (2.11) and (2.13) into equations (2.8), we may write

$$
\sigma_{r}=\sigma_{r}^{E}+\bar{\sigma}_{r}-\sigma_{r}
$$




$$
\sigma_{t}=\sigma_{t}^{E}+\bar{\sigma}_{t}-\sigma_{t}^{\prime \prime}
$$

where

$$
\begin{aligned}
& \sigma_{r}^{E}=\frac{N_{r}^{E}}{h}+\frac{12 z}{h^{3}} \frac{E}{r}_{r} \\
& \sigma_{t}^{E}=\frac{N_{t}^{E}}{h}+\frac{12 z}{n^{3}} \frac{E}{t}_{t}
\end{aligned}
$$

and

$$
\begin{aligned}
& \bar{\sigma}_{r}=\frac{\bar{N}_{r}}{h} \\
& \bar{\sigma}_{t}=\frac{\bar{N}_{t}}{h} \\
& \sigma_{r}^{\prime \prime}=\frac{E}{1-v^{2}}\left(\varepsilon_{r}^{\prime \prime}+v \varepsilon^{\prime \prime}\right) \\
& \sigma_{t}^{\prime \prime}=\frac{E}{1-v^{2}}\left(\varepsilon_{t}^{\prime \prime}+v \varepsilon^{\prime \prime}\right)
\end{aligned}
$$


GOVERNING EQUILIBRIUM EQUATIONS

Let us now consider the equilibrium of an element of the plate, such as element abcd shown in Fig. 4 a \& b, cut out from the plate by two cylindrical sections $a b$ and $c d$ and by two diametral sections ad and bc. Taking the sum of the projections in the radial direction of all the forces acting on the element we obtain

$$
\left(N_{r}+N_{r, r} d r\right)(r+d r) d t-N_{r} r d t-2 N_{t} d r \frac{d t}{2}=0
$$

neglecting $N_{r}, r$ drdrdt as a small quantity of higher order, the first equation of equilibrium may be stated as

$$
N_{r, r}+\frac{N_{r}-N_{t}}{r}=0
$$

The second equation of equilibrium of the element is obtained by taking moments of all the forces acting on the element with respect to an axis perpendicular to the radius in the roz plane shown in Fig. 4b. Neglecting the higher order terms resulting from $q$ and $a_{r}$, we obtain

$$
\left(M_{r}+M_{r, r} d r\right)(r+d r) d t-M_{r} r d t-Q_{r} r d r d t-2 M_{t} d r \frac{d t}{2}=0
$$

Disregarding $M_{r}, r d r d r d t$ as a small quantity of higher order, the second equation of equilibrium may be stated as 


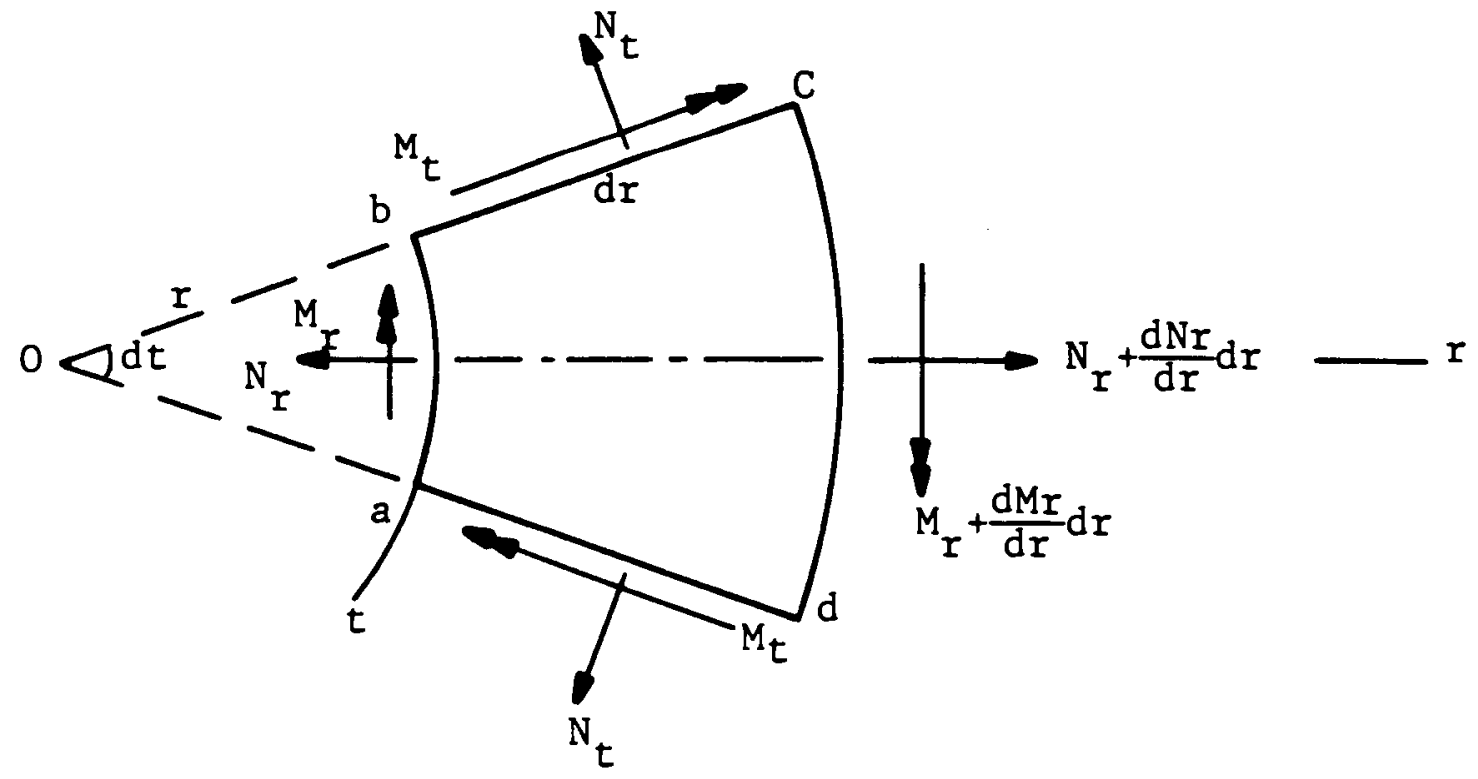

(a) PLATE ELEMENT (TOP VIEW)

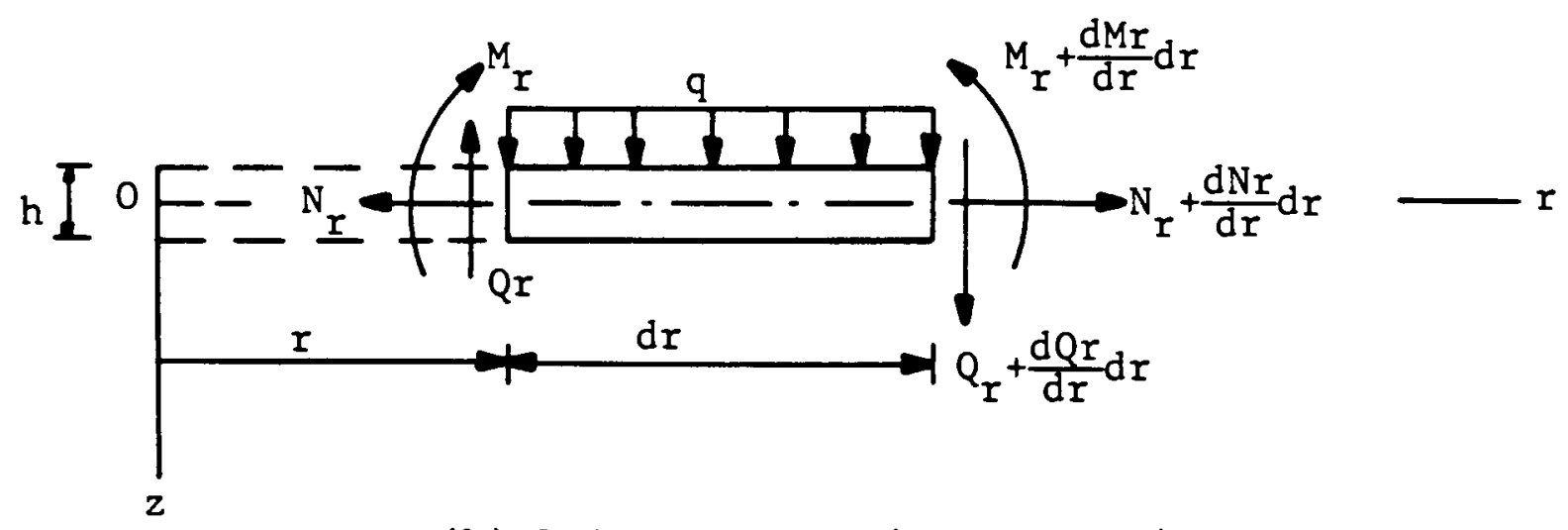

(b) PLATE ELEMENT (FRONT VIEW)

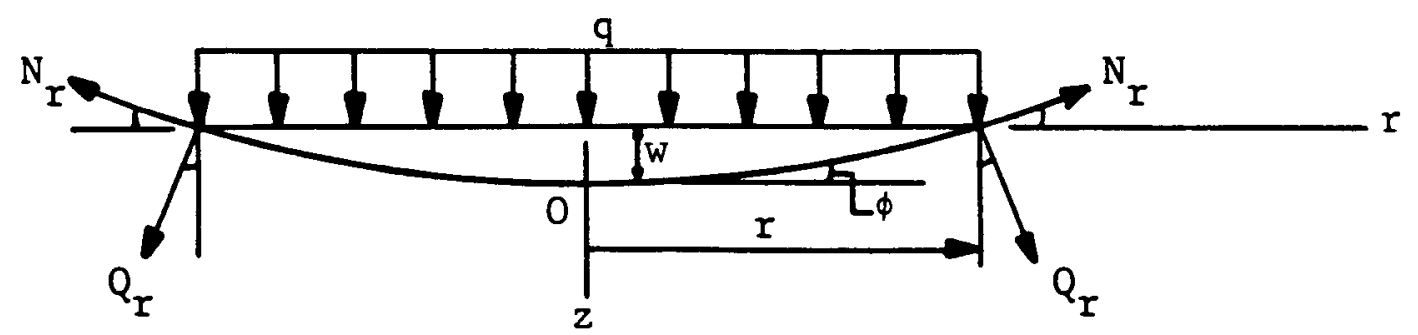

(c) INNER CIRCULAR PORTION OF RADIUS $r$

FIGURE 4. EQUILIBRIUM OF A CIRCULAR PLATE 


$$
M_{r, r}+\frac{M_{r}-M_{t}}{r}-Q_{r}=0
$$

Denoting the intensity of the load by $q$, the magnitude of the shearing force $a_{r}$ is evaluated by considering the equilibrium of an inner circular portion of the plate of radius $r$ shown in Fig. 4c. For a uniform pressure such a consideration yields the relation

$$
Q_{r}=-\frac{q r}{2}-N_{r}{ }{ }_{r}
$$

Substituting the expression for the shearing force $Q_{r}$ into expression (2.25), the second equation of equilibrium may be rewritten as

$$
M_{r, r}+\frac{M_{r}-M_{t}}{r}+N_{r} w_{r}+\frac{q r}{2}=0
$$

Equation (2.23) may expressed in terms of displacements by using the first two parts of relations $(2.10)$ and (2.11). The first equation of equilibrium becomes

$$
\frac{E h}{1-v^{2}}\left(u_{0, r r}+\frac{1}{r} u_{0, r}-\frac{1}{r^{2}} u_{0}\right)+\bar{R}(r)+R "(r)=0
$$

where

$$
\bar{R}(r)=\bar{N}_{r, r}+\frac{\bar{N}_{r}-\bar{N}_{t}}{r}
$$




$$
R^{\prime \prime}(r)=-\left(N_{r, r}^{\prime \prime}+\frac{\bar{N}_{r}-\bar{N}_{t}}{r}\right)
$$

In a similar manner, the second equation of equilibrium may be written in terms of the middle surface displacement by substituting equations (2.10) and $(2.11 . c \& d)$ in $(2.27)$. We then have

$$
D \nabla^{4} w=q+\bar{q}(r)+q^{\prime \prime}(r)
$$

where

$$
\begin{aligned}
& \nabla^{4} w=w, \underset{r r r r}{r}+\frac{2}{r r r}-\frac{1}{r^{2}} w_{r r}+\frac{1}{r^{3}} w r \\
& \bar{q}(r)=\frac{1}{r} N_{r} w_{r}+N_{r, r}{ }^{w}, r+N_{r} w_{r r} \\
& q^{\prime \prime}(r)=-\frac{1}{r}\left(2 M_{r, r}^{\prime \prime}-M_{t, r}^{\prime \prime}+r M_{r, r r}^{\prime \prime}\right)
\end{aligned}
$$

Equations (2.28) to (2.32) may be rewritten in terms of the plate displacements and plastic strains by making use of relations (2.13) and (2.14). The resulting governing equations are

$$
\frac{E h}{1-v^{2}}\left(u_{0, r r}+\frac{1}{r} u_{0, r}-\frac{1}{r^{2}} u_{0}\right)+\bar{R}(r)+R(r)=0
$$




$$
D \nabla^{4} w=q+\bar{q}(r)+q^{\prime \prime}(r)
$$

where

$$
\begin{aligned}
& \bar{R}(r)=\frac{E h}{1-\nu^{2}}\left[w, w^{,}, r r+\frac{(1-\nu)}{2 r} w^{2}, r\right] \\
& \bar{q}(r)=\frac{1}{r} N_{r} w_{r}+N_{r, r}{ }^{,}, r+N_{r}{ }^{,}, r r \\
& R^{\prime \prime}(r)=-\frac{E}{1-\nu^{2}}\left[\frac{d}{d r} \int\left(\varepsilon_{r}^{\prime \prime}+v \varepsilon_{t}^{\prime \prime}\right) d z+\frac{(1-v)}{r} \int\left(\varepsilon_{r}^{\prime \prime}-\varepsilon_{t}^{\prime \prime}\right) d z\right] \\
& q^{\prime \prime}(r)=-\frac{E}{1-v^{2}} \frac{1}{r} \frac{d}{d r}\left[r \frac{d}{d r} \int\left(\varepsilon_{r}^{\prime \prime}+v \varepsilon_{t}^{\prime \prime}\right) z d z+(1-v) \int\left(\varepsilon_{r}^{\prime \prime}-\varepsilon_{t}^{\prime \prime}\right) z d z\right](2.35 . b)
\end{aligned}
$$

Expressions (2.33-2.35) show that the nonlinear effects of finite lateral displacement and the plastic strains are given by $\bar{R}, \bar{q}$ and $R$," $q$," respectively. It is al so seen that $\bar{R}, R^{\prime \prime}$ have the same effect in causing displacements as the in-plane radial body forces. Thus $\bar{R}, R^{\prime \prime}$ may be considered as equivalent radial body forces. In a similar fashion, $\bar{q}$ and $q$ "have the same effect in causing lateral deflections as the applied lateral load $q$ and, hence, $\bar{q}, q$ " may be treated as equivalent lateral loads. Therefore, relations (2.33) are identical to the governing equations for small deformation of an elastic thin plate subjected to a radial body force $\bar{R}+R^{\prime \prime}$ and a lateral load of magnitude $q+\bar{q}+q$. The solution of an 
elastic or elasto-plastic Von Karman type plate can, therefore, be reduced to that of an equivalent elastic plate subjected to additional body and surface forces and undergoing small displacements. 
CHAPTER III

PLASTIC STRESS-STRAIN RELATIONS

The fundamental problem in applying plasticity theory is to determine the total plastic strain as a function of the history of loading or history of stress. To calculate the plastic strains at a final load condition, it is necessary to integrate the infinitesimal plastic strain increments over the actual loading path $(2,53-55)$. This may be accomplished by applying the load in small finite increments and calculating the finite increments of plastic strain for each of the load increments. All these increments of plastic strain are then added to give the total plastic strain.

Let the total loading path be divided into $n$ increments of load. Assume that the plastic strains have been computed for the first $n-1$ increments of load and we now wish to compute them for the $n^{\text {th }}$ increment of load. The total strains at the end of the $n^{\text {th }}$ increment can be written as

$$
\varepsilon_{i j}=\stackrel{e}{\varepsilon}_{i j}+{ }_{k=1}^{n-1} d \varepsilon_{i j, k}+d \varepsilon_{i j, n} " \quad(i, j=1,2,3)
$$

where the first term on the right side of equation (3.1) represents the elastic part of the total strain, the second term is the plastic strain accumulated in the first $n-1$ increments of load, and the third term is the 
plastic strain increment due to the $n^{\text {th }}$ increment of load. In the above equation the sum is assumed to be known and the problem is to calculate the plastic strain increment for the current or $n^{\text {th }}$ increment of load and its corresponding stresses.

The first step of any plastic flow analysis is to decide on a yield criterion, i.e., what combination of multiaxial stresses will cause yielding. Beyond the elastic limit, a set of incremental plastic stress-strain relations are needed in order to describe the behavior of the material after yielding has occured.

In the most general case, the yield criterion will depend on the complete state of stress at the point under consideration and will therefore be a function of the nine components of stress at the point. Since the stress tensor is symmetric, we can reduce this function to a function of the six independent components of the stress tensor. The yield criterion for a virgin material is then essentially the extension of the single yield point of the uniaxial tensile test to the six-component stress tensor. For a material loaded to the initial yield, it can be expressed by the relationship

$$
F\left(\sigma_{i j}\right)=k \quad(i, j=1,2,3)
$$

Equation (3.2) represents a convex hypersurface in the six-dimensional stress space and any point on this surface represents a point at which yielding can begin. For example, for the simple tensile test with a yield point $\sigma_{y}$, the point $\sigma_{11}=\sigma_{y}, \sigma_{22}=\sigma_{33}=\sigma_{12}=\sigma_{13}=\sigma_{23}=0$ must lie on this surface. The function appearing in equation (3.2) is called the yield 
function, and the surface described by it in the stress space is called the yield surface.

If isotropy is assumed so that the rotation of the axes does not affect the yielding function, equation (3.2) $c$ an be expressed in terms of the principal stresses as

$$
F_{1}\left(\sigma_{1}, \sigma_{2}, \sigma_{3}\right)=k
$$

Based on extensive experimental studies, it has been found that hydrostatic pressure has negligible effect on the yield point until extremely high pressures are reached. It has also been shown that the density, and consequently the volume, does not change for very large plastic deformations. Thus, in the plastic range a material can be considered as incompressible. The yield function may then be expressed in terms of the stress deviators as

$$
F_{2}\left(S_{1}, S_{2}, S_{3}\right)=k
$$

Alternatively, $S_{1}, S_{2}$, and $S_{3}$ can be written in terms of the invariants of the stress deviator tensor. Since the first invariant of this tensor is always zero, we can write

$$
F_{3}\left(J_{2}, J_{3}\right)=k
$$

Subject therefore to the above assumption, the yield criterion has been reduced to a function of the two nonzero invariants of the stress deviator tensor (55). 
The two most widely used criteria, the Tresca maximum shear criterion and the Von Mises criterion, are specific cases of equation (3.5). The Von Mises criterion is by far the simplest one that can be associated with equation (3.5) and is, therefore, the initial yield criterion utilized in this study.

\section{VON MISES INITIAL YIELD CRITERION}

Von Mises (1913) stated that initial yielding will occur when the second invariant $J_{2}$ of the stress deviator tensor reaches a critical value, i.e., the value of $J_{2}$ at yield in simple tension.

Let $x_{i}(i=1,2,3)$ refer to a set of Cartesian coordinate system. The components of stress with respect to $x_{i}$ are denoted by $\sigma_{i j}(i, j=$ $1,2,3)$. For this general state of stress, $J_{2}$ is given by

$$
J_{2}=\sigma_{12}^{2}+\sigma_{23}^{2}+\sigma_{13}^{2}+\frac{1}{6}\left[\left(\sigma_{11}-\sigma_{22}\right)^{2}+\left(\sigma_{22}-\sigma_{33}\right)^{2}+\left(\sigma_{33}-\sigma_{11}\right)^{2}\right]
$$

At the yield point in simple tension, equation (3.6) reduces to

$$
J_{2}=\frac{1}{3} \sigma_{y}^{2}
$$

As a result, the Von Mises initial yield criterion may be mathematically expressed in the following form

$$
\sigma_{12}^{2}+\sigma_{23}^{2}+\sigma_{13}^{2}+\frac{1}{6}\left[\left(\sigma_{11}-\sigma_{22}\right)^{2}+\left(\sigma_{22}-\sigma_{33}\right)^{2}+\left(\sigma_{33}-\sigma_{11}\right)^{2}\right]=\frac{1}{3} \sigma_{y}^{2}(3.8)
$$


Specializing this equation to an axisymmetrically loaded circular or annular plate in a state of plane stress, the yield criterion may be expressed in terms of radial and tangential stresses as

$$
\sigma_{r}^{2}+\sigma_{t}^{2}-\sigma_{r} \sigma_{t}=\sigma_{y}^{2}
$$

which plots as an ellipse, called the Von Mises ellipse, in the $\sigma_{r} \sigma_{t}$ plane as shown in Fig. 5 . It is worth noting that the Von Mises yield criterion implies equal yielding in tension and compression.

The Von Mises criterion of initial yielding was first proposed due to its simplicity and mathematical convenience. Nevertheless, the famous experiments of Taylor and Quininney (1921) along with most other test results have shown this criterion to exhibit good correlation with experimental data $(2,53-55)$. Attempts have been made to improve the correlation of the data by including the effect of the third invariant $\mathrm{J}_{3}$ into the yield criterion (66). It seems, however, that from an engineerng viewpoint the accuracy of the Von Mises criterion for yielding is amply sufficient when considering the general scatter and lack of uniformity in the properties of nominally the same material obtained from different batches. For these reasons, this criterion is widely utilized in the inelastic analysis of metals.

\section{SUBSEQUENT YIELD SURFACES}

Thus far, the discussion has been confined to the initial yield 


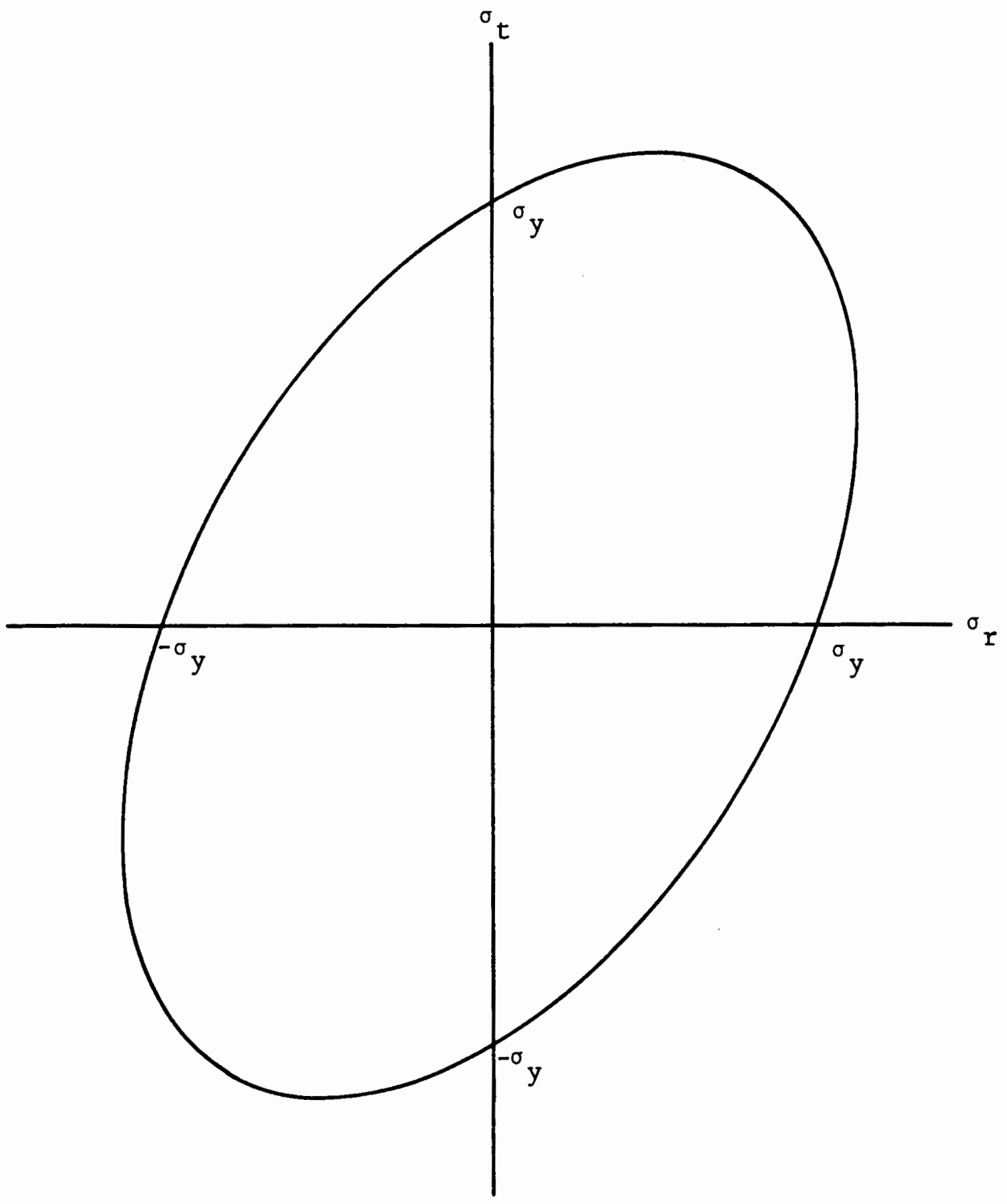

FIGURE 5. GRAPHICAL REPRESENTATION OF THE VON MISES INITIAL YIELD CRITERION FOR AXISYMMETRICALLY LOADED PLATES 
surface at which a material will first start yielding. For a perfectly plastic material, the yield surface remains fixed, as seen from the uniaxial tensile test data where the stress after yielding remains constant at the initial yield. However, for a material that strain hardens, such as aluminum, the yield surface must change for continued straining beyond the initial yield. In equation (3.2) rewritten below for convenience, the yield function was defined by the relation

$$
F\left(\sigma_{i j}\right)=k \quad(i, j=1,2,3)
$$

such that whenever the function $F$ became equal to the constant $k$, yielding would begin. Then $k$ represented an initial yield surface in the stress space. This type of relation can now be generalized to subsequent yield surfaces.

After yielding has occured, $k$ takes on a new value depending on the strain-hardening properties of the material. If the material is unloaded and then loaded again, additional yielding will not occur until the new value of $k$ is reached. The function $F$ can then be looked upon as a loading function which represents the load being applied, and the function $k$ is a yield function which is dependent on the complete previous stress and strain history of the material and its strain-hardening properties. Three distinct cases may be encountered for a strain-hardening material:

(1) $\quad F=k$ and $d F=\frac{\partial F}{\partial \sigma_{i j}} d \sigma_{i j}>0$ 
This case constitutes loading

(2) $\quad F=k$ and $d F=\frac{\partial F}{\partial \sigma_{i j}} d \sigma_{i j}=0$

This is called neutral loading

(3) $\quad F=k$ and $d F=\frac{\partial F}{\partial \sigma_{i j}} d \sigma_{i j}<0$

Which defines the unloading. The following geometric interpretation of the conditions expressed by relations (3.11) is readily evident: $F=k$ means that the stress state is on the yield surface. $d F>0$, on the other hand, describes the case where the stress is "moving out" from the yield surface and plastic flow is occuring, while $\mathrm{dF}<0$ refers to a state when the stress is "moving in" from the yield surface and unlaoding is therefore taking place. $d F=0$ corresponds to the case of the stress state moving on the yield surface and is called neutral loading. For a strain-hardening material no plastic flow occurs for neutral loading. It is obvious that $F$ $<k$ defines the elastic region.

There is one more ingredient necessary in constructing the plasticity theory, namely, the relations between stress and strain when plastic flow is occuring. In this study the incremental plastic strains are obtained by utilizing the Prandtl-Reuss polyaxial inelastic stress-strain relations. 
PRANDTL-REUSS INCREMENTAL PLASTIC

STRESS-STRAIN RELATIONS

In 1870 Saint Venant proposed that the principal axes of strain increments coincide with the axes of principal stresses. Levy (1871) and subsequently Von Mises (1913) introduced the general relations between strain increments and the deviatoric stresses. Prandt1 (1924) and Reuss (1930) assumed that the plastic strain increment tensor is, at any instant of loading, proportional to the instantaneous deviated stress tensor, expressed as

$$
\frac{d \varepsilon_{i j}}{S_{i j}}=d \lambda \quad(i, j=1,2,3)
$$

where $d \lambda$ is an instantaneous positive constant of proportionality which may vary during the loading process and $S_{i j}$ are the deviatoric stress components. $\mathrm{d} \lambda=0$ for unloading and neutral loadings.

Equation (3.12) states that the increments of plastic strain depend on the current values of the deviatoric stress state, not on the stress increments required to reach this state. The relation also implies that the principal axes of stress and of plastic strain increment tensors coincide. Furthermore, the condition of zero plastic dilitation is satisfied. For an axisymmetrically loaded circular or annular plate, equation (3.12) may be written as

$$
d \varepsilon_{r}^{\prime \prime}=S_{r} d \lambda
$$




$$
d \varepsilon_{t}^{\prime \prime}=S_{t} d \lambda
$$

where the radial and tangential deviatoric stress components are given by

$$
\begin{aligned}
& S_{r}=\frac{2 \sigma_{r}-\sigma_{t}}{3} \\
& S_{t}=\frac{2 \sigma_{t}-\sigma_{r}}{3}
\end{aligned}
$$

Substituting equations (3.14) into equations (3.13), the incremental plastic stress-strain relations can be written in terms of the radial and tangential stresses as

$$
\begin{aligned}
& \mathrm{d}_{r}^{\prime \prime}=\frac{2 \sigma_{r}-\sigma_{t}}{3} \mathrm{~d} \lambda \\
& \mathrm{d}_{\mathrm{t}}^{\prime \prime}=\frac{2 \sigma_{\mathrm{t}}-\sigma_{r}}{3} \mathrm{~d} \lambda
\end{aligned}
$$

Once the value of $d \lambda$ is known, the desired stress-strain relations are defined. To determine $d \lambda$, let us define effective stress $\stackrel{\star}{\sigma}$ and and effective plastic strain increment $\stackrel{\star \star \prime \prime}{d}$ as

$$
\begin{aligned}
& \stackrel{\star}{\sigma}=\left(\sigma_{r}^{2}+\sigma_{t}^{2}-\sigma_{r} \sigma_{t}\right)^{1 / 2} \\
& d \stackrel{\star \prime \prime}{\varepsilon}=\frac{2}{\sqrt{3}}\left(d \varepsilon_{r}^{2}+d \varepsilon_{t}^{\prime \prime 2}+d \varepsilon_{r}^{\prime \prime} d \varepsilon_{t}^{\prime \prime}\right)^{1 / 2}
\end{aligned}
$$


For a uniaxial tensile state of stress in the radial direction, the effective stress $\stackrel{\star}{\sigma}$ reduces to $\sigma_{r}$. Utilizing the incompressibility assumption, i.e., $d \varepsilon_{t}^{\prime \prime}=d \varepsilon_{z}^{\prime \prime}=-\frac{1}{2} d \varepsilon_{r}^{\prime \prime}$ the effective plastic strain increment $d \varepsilon^{\star \prime \prime}$, likewise, reduces to $d \varepsilon_{r}^{\prime \prime}$. For the uniaxial state of stress, equation (3.15.a) becomes

$$
d E_{r}^{\prime \prime}={ }_{3}^{2} \sigma_{r} d \lambda
$$

Generalization of the above case to a multiaxial state of stress results in

$$
d \stackrel{\star}{\varepsilon}=\frac{2}{3} \stackrel{\star}{\sigma} \mathrm{d} \lambda
$$

The constant $d \lambda$ can now be written as

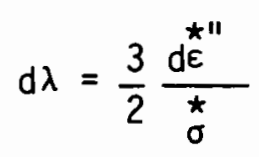

And the stress-strain relations (3.15) become

$$
\begin{aligned}
& d \varepsilon_{r}^{\prime \prime}=\left(\sigma_{r}-\frac{1}{2} \sigma_{t}\right) \frac{d \varepsilon^{\star \prime \prime}}{\underset{\sigma}{\star}} \\
& d \varepsilon_{t}^{\prime \prime}=\left(\sigma_{t}-\frac{1}{2} \sigma_{r}\right) \frac{d \varepsilon^{\star \prime}}{\sigma_{\sigma}^{\star}}
\end{aligned}
$$


The generalization of the uniaxial stress-strain relation to a multiaxial stress state is defined by $\stackrel{\star *}{\varepsilon}=g(\stackrel{*}{\sigma})$. For strain-hardening aluminum, this function is approximated by (67)

$$
\stackrel{\star \star n}{\varepsilon}=\left(\frac{\stackrel{*}{\sigma}-\sigma_{y}}{\sigma_{0}}\right)^{n}
$$

where $\sigma_{0}$ and $n$ are constants.

And hence

$$
\begin{aligned}
& \left.d \stackrel{\star}{\varepsilon}^{\prime \prime}=\frac{n}{\sigma}{\stackrel{\star \star}{\sigma}-\sigma_{y}}_{0}\right)^{n-1} d \stackrel{*}{\sigma} \text { when } \stackrel{\star}{\sigma}>\sigma_{y} \\
& d \stackrel{\star \prime \prime}{\varepsilon}=0 \text { when } \stackrel{\star}{\sigma}\left\langle\sigma_{y} \text { or } \stackrel{\star}{\sigma}=\sigma_{y}\right.
\end{aligned}
$$

Substituting equation (3.23.a) into equations (3.21), the incremental plastic stress-strain relations for axisymmetrically loaded plates made of strain-hardening aluminum may be expressed as

$$
\begin{aligned}
& d \varepsilon_{r}^{\prime \prime}=\frac{n}{\sigma_{0}}\left(\sigma_{r}-\frac{1}{2} \sigma_{t}\right)\left(\frac{{\stackrel{\sigma}{\sigma}-\sigma_{y}}_{\sigma}}{\sigma_{0}}\right)^{n-1} \frac{d \sigma^{*}}{\frac{\star}{\sigma}} \\
& d \varepsilon_{t}^{\prime \prime}=\frac{n}{\sigma_{0}}\left(\sigma_{t}-\frac{1}{2} \sigma_{r}\right)\left(\frac{\stackrel{*}{\sigma}-\sigma_{y}}{\sigma_{0}}\right)^{n-1} \frac{d \sigma^{*}}{\frac{\sigma}{\sigma}}
\end{aligned}
$$


where

$$
\stackrel{\star}{\sigma}=\left(\sigma_{r}^{2}+\sigma_{t}^{2}-\sigma_{r} \sigma_{t}\right)^{1 / 2}
$$

hence

$$
d \sigma^{\star}=\frac{1}{\star}\left[\left(\sigma_{r}-\frac{1}{2} \sigma_{t}\right) d \sigma_{r}+\left(\sigma_{t}-\frac{1}{2} \sigma_{r}\right) d \sigma_{t}\right]
$$

and $d \varepsilon_{r}^{\prime \prime}=d \varepsilon_{t}^{\prime \prime}=0 \quad$ when $d \stackrel{\star}{\sigma}<0$

It is worth noting that the Prandtl-Reuss assumptions imply the Von Mises yield criterion. This can readily be seen by comparing the equation defining the effective stress (3.25.a) with equation (3.9), which gives the Von Mises yield criterion. Such a comparison shows that yielding will initiate when

$$
\stackrel{\star}{\sigma}=\sigma_{y}
$$

The equivalent stress is thus the same as the Von Mises yield function and, therefore, the Von Mises initial yield criterion may be restated as: initial yielding will occur when the effective stress reaches a critical value, i.e., the value of the yield stress in simple tension. 
CHAPTER IV

METHOD OF SOLUTION

In the earlier discussion, it was stated that the inelastic strains as well as the nonlinear terms of lateral displacement can be considered as a combination of lateral loads, edge moments, and in-plane forces acting on an equivalent plate. The solution of an elastic or elasto-plastic Von Karman type circular or annular plate is, therefore, reduced to that of an equivalent elastic plate subjected to additional body and surface forces with small displacements. The incremental plastic stress-strain relations (3.25) are employed in this analysis. The displacement, stress, and strain fields are obtained by the solution of the governing equilibrium equations (2.33) together with the constitutive relations (2.10) in their incremental forms.

A general discussion of the equivalent load theory (2) as it relates to circular and annular plates undergoing large deflections, along with a detailed account of the incremental numerical scheme utilized to solve the nonlinear set of governing equilibrium equations are presented herein.

\section{EQUIVALENT LOAD THEORY}

The well known analogy between thermal strains and applied forces (Duhamel's Analogy) was first generalized by $L$ in $(41,62)$ to include creep 
and plastic strains. Lin (2) showed that the lateral deflections and stresses in an inelastic simply supported plate undergoing small deflections may be calculated by considering the inelastic strains as an additional set of lateral loads and edge moments acting on an equivalent elastic plate. Lackman (40) employed the analogy between inelastic strains and applied lateral loads to obtain the solution for a simply supported elasto-plastic circular plate with small deflections. Later, Lin $(61,63)$ extended the concept to obtain the large deflection solutions to elasto-plastic and creep bending problems of isotropic rectangular plates. Recently, Gorji $(1,60)$ has used this approach to obtain solutions for elastically isotropic-plastically orthotropic rectangular plates as well as symmetric composite plates with large deflections. The present study utilizes this method to obtain the elastic and elasto-plastic solutions to circular and annular plates undergoing large deflections.

Accordingly, the equivalent load concept is used in the analysis of large deflection (in the Von Karman sense) of elastic and elasto-plastic circular plates with or without a hole at the center. The nonlinear terms of lateral displacement and the plastic strains are considered as an additional set of loads acting on the plate. The solution of an elastic or elasto-plastic Von Karman type plate is hence reduced to a set of two equivalent elastic plate problems with small displacements, namely, a plane problem in elasticity and a linear plate bending problem. Examination of equation (2.33.a) showed that the nonlinear effects of finite lateral displacement and the inelastic strains have the same effect in causing 
radial displacement as the in-plane body force $R$. Thus $\bar{R}$ and $R$ " were called equivalent radial body forces due to finite lateral displacement and the plastic strains, respectively. In a similar fashion, equation (2.33.b) indicated that the equivalent lateral forces $\bar{q}$ and $q$ " have the same influence in causing lateral deflections as the applied load $q$, and therefore were considered as equivalent lateral loads induced by these nonlinear effects. Hence, if the displacements are prescribed at the boundaries of the plate, the deformation of a Von Karman type plate under the action of a lateral load $\mathbf{q}$ would be the same as that of an equivalent plate undergoing small displacements and subjected to a radial body force $\bar{R}+R^{\prime \prime}$, and a lateral load $q+\bar{q}+q^{\prime \prime}$. This is true because the governing equations (2.33) and the boundary conditions are identical for both cases. On the other hand, if forces are prescribed on some portion of the boundary, the same conclusion can still be reached provided the corresponding boundary forces of the equivalent plate are modified to reflect the nonlinear terms of lateral displacement and the plastic strains given by equations (2.13), (2.14), and (2.17.b).

Since the radial force $N_{r}$ as well as the moment $M_{r}$ and Kirchhoff shear $V_{r}$ (equivalent to $Q_{r}$ for an axisymmetric plate) are the prescribed at the inner free edge of the annular plates considered in this study, the corresponding forces acting on the inner edge of the equivalent $\mathrm{plate}$ are modified in order to account for the nonlinear terms of displacement and the effect of the plastic strains present in equations $(2.10 . a),(2.10 . c)$ and (2.16). In a similar manner, the moment $M_{r}$ at the outer boundary of the simply supported equivalent plate is modified in 
order to reflect the prescribed moment at the corresponding boundary of the actual plate. For a clamped plate, however, displacements are prescribed at its outer boundary and no modification of the boundary conditions of the equivalent plate is needed. The actual and corresponding equivalent boundaries for the three classes of circular boundaries discussed above are shown in Fig. 6 .

The sectional forces and stresses in the equivalent plate may be calculated from the displacement field as can be readily seen by examining expressions (2.11) and (2.19). Therefore, the influence coefficients for radial and lateral nodal displacements due to unit body and surface forces must be known in order to evaluate the sectional forces and stresses in the equivalent plate. Since the equivalent plate is elastic, the method of superposition is utilized to obtain the nodal displacements due to body and surface forces of various magnitudes.

While the influence coefficients of elastic plate bending problems under arbitrary surface loading conditions are generally known $(3,65$, Appendix $B$ ), the influence coefficients for the plane stress problems of finite region under body forces are not always available. However, with the use of numerical techniques such as the finite element method (64), the influence coefficients of elastic plane stress problems under arbitrary body loading conditions are obtainable. In this study, a finite element program (SAP IV) was utilized to obtain the influence coefficients due to unit radial in-plane forces.

The stresses in the Von Karman type plate may be obtained by superposing on the stresses obtained from the corresponding equivalent 

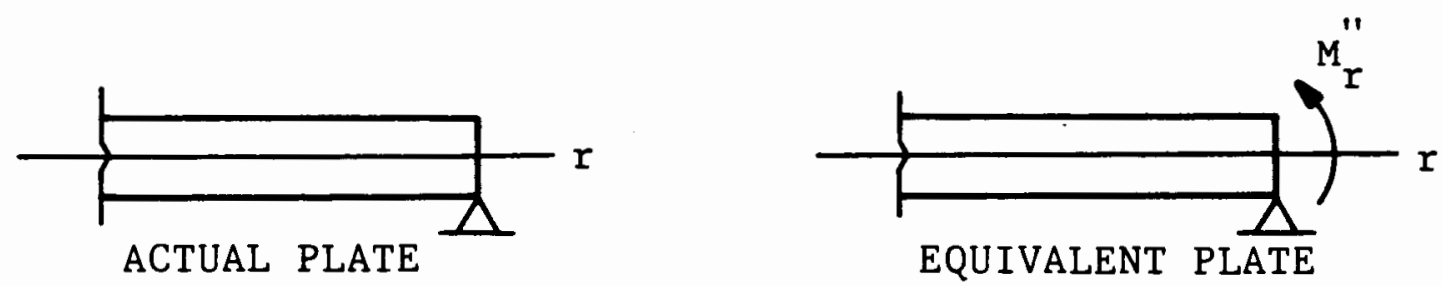

(a) SIMPLY SUPPORTED BOUNDARY

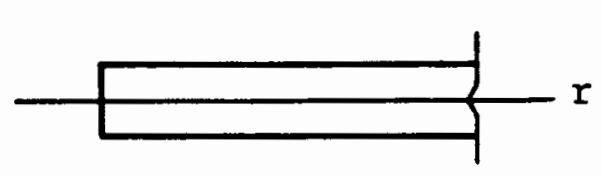

ACTUAL PLATE

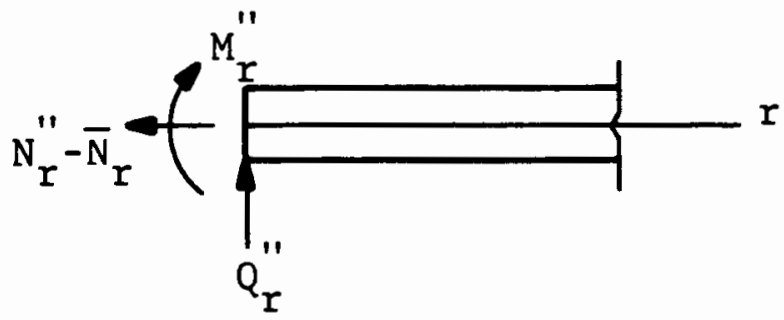

EQUIVALENT PLATE

(b) FREE BOUNDARY

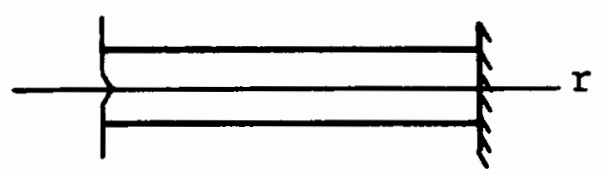

ACTUAL PLATE

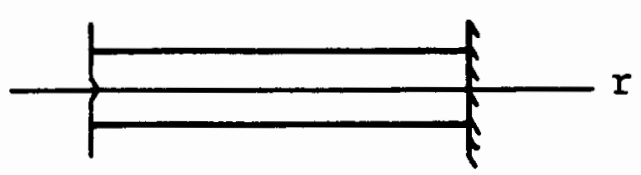

EQUIVALENT PLATE

(c) CLAMPED BOUNDARY

FIGURE 6. ACTUAL AND CORRESPONDING EQUIVALENT PLATE BOUNDARIES 
plate solutions, the stresses arising from the nonlinear terms of lateral displacement and the plastic strains as seen from constitutive relations (2.10) and (2.18).

Due to the symmetry of the loading and boundaries about the plate axis, the plane of the plate is divided into a number of equal segments along a typical radial direction. Moreover, the variation of the plastic strain in the thickness direction is considered by dividing the plate into four equal layers along its thickness. The method discussed earlier is then used to solve the governing equations of equilibrium in their incremental forms in order to obtain the displacement, stress, and strain fields. The iteration technique utilized in the solution procedure is discussed in the following section.

\section{INCREMENTAL NUMERICAL SCHEME}

Consider a circular or annular plate with arbitrary boundary conditions subjected to a uniformly distributed load $q$. Let this load be increased by equal increments $\Delta q$ until the desired load is reached. The increments of lateral displacement, plastic strain, stress, etc. are denoted by double subscribed notations, where the first subscript is associated with the load increment and the second subscript denotes the cycle of iteration. Thus, $\Delta w(n+1) m^{\text {and }} \Delta \varepsilon_{i j(n+1) m}^{\prime \prime}$ refer, respectively, to the increments of lateral displacement and plastic strain due to the $(n+1)^{\text {th }}$ load increment at the end of the $m^{\text {th }}$ iteration cycle. When the final values of these variables are reached for any load increment, the 
second subscript is deleted. Assume that the increments of lateral displacement and plastic strain due to the $(n+1)^{\text {th }}$ increment of load are equal to those obtained at the end of the $n^{\text {th }}$ load increment. This indicates that

$$
\begin{aligned}
\Delta w_{(n+1) 1} & =\Delta w_{(n)} \\
\Delta \varepsilon_{r(n+1) 1}^{\prime \prime} & =\Delta \varepsilon_{r(n)}^{\prime \prime} \\
\Delta \varepsilon_{t(n+1) 1}^{\prime \prime} & =\Delta \varepsilon_{t(n)}^{\prime \prime}
\end{aligned}
$$

and

$$
w_{(n+1) 1}=w_{(n)}+\Delta w_{(n+1) 1}
$$

Substituting these assumed values in relations (2.34.a) and (2.35), a trial set of values for of the equivalent radial body forces $\Delta \bar{R}(n+1) 1, \Delta R^{\prime \prime}(n+1) 1$ and lateral load $\Delta q^{\prime \prime}(n+1) 1$ are obtained. Note that the magnitude of $\Delta \bar{q}(n+1) 1$ is not known at this time. With the trial values of of $\Delta \bar{R}(n+1) 1$ and $\Delta R "(n+1) 1$ known, equation (2.33.a) is solved for the in-plane radial displacement increment of $\Delta u_{0}(n+1) 1$. This equation represents an elastic plane stress problem for which influence coefficients have been obtained by the method of finite element. The membrane forces $\Delta N_{r}(n+1) 1$ and $\Delta N_{t}(n+1) 1$ are evaluated by substituting the incremental in-plane displacement along with the assumed values of incremental lateral displacement and incremental plastic strain into constitutive relations 
(2.10.a) and (2.10.b). The sectional forces $N_{r}(n+1) 1$ and $N_{t}(n+1) 1$ are then obtained by

$$
\begin{aligned}
& N_{r(n+1) 1}=N_{r(n)}+\Delta N_{r(n+1) 1} \\
& N_{t(n+1) 1}=N_{t(n)}+\Delta N_{t(n+1) 1}
\end{aligned}
$$

having obtained the in-plane radial forces, the lateral load $\Delta \bar{q}(n+1) 1$ and consequently $\bar{q}(n+1) 1$ may now be determined from relation (2.34.b). Using the trial values of lateral displacement, plastic strain and in-plane displacement in relations (2.24), stresses $\Delta \sigma_{r(n+1) 1}, \Delta \sigma_{t(n+1) 1}$ and hence $\sigma_{r(n+1) 1}, \sigma_{t(n+1) 1}$ are determined. The incremental plastic stress-strain relations (3.24) are subsequently utilized to evaluate a new set of values of incremental plastic strains $\Delta \varepsilon_{r(n+1) 2}^{\prime \prime}$ and $\Delta \varepsilon_{t(n+1) 2}^{\prime \prime}$. Inserting the values of $\Delta \bar{q}(n+1) 1, \Delta q "(n+1) 1$, and the applied load increment $\Delta q$ in equation (2.33.b) allows for the determination of $\Delta w(n+1) 2$. This is a linear elastic plate bending problem for which influence coefficients have been obtained using analytical solutions (Appendix B). With the new calculated values of $\Delta w(n+1) 2$ and $\Delta \varepsilon_{r}^{\prime \prime}(n+1) 2, \Delta \varepsilon_{t}^{\prime \prime}(n+1) 2$ known, the same procedure is repeated until the difference between the successive values of $\Delta w, \Delta \varepsilon_{r}^{\prime \prime}$, and $\Delta \varepsilon_{t}^{\prime \prime}$ are within the desired tolerances. In this way the lateral displacements, stresses, and strains of Von Karman type circular and annular plates are obtained. 


\section{NUMERICAL SOLUTION CONVERGENCE \\ ACCURACY AND STABILITY}

The convergence process was significantly enhanced by assuming new trial values of lateral displacement and plastic strains based on a linear combination of the trial and calculated values obtained in the preceding iteration cycle. A numerical scheme used for that purpose is presented in the following.

Let $\Delta w_{(n) m-1}, \Delta \varepsilon_{i j(n) m-1}^{\prime \prime}$ and $\Delta w(n) m, \Delta \varepsilon_{i j(n) m}$, denote, respectively, the trial and calculated values of the incremental lateral displacement and incremental plastic strain components at the $m^{\text {th }}$ cycle of iteration due to the $n^{\text {th }}$ loading increment. Furthermore, assume that the difference between these successive values of $\Delta w$ and $\Delta \varepsilon_{i j}$ are not within the desired limit so that further iteration is required. The trial values of $\Delta w$ and $\Delta \varepsilon_{i j}^{\prime \prime}$ for the $(m+1)^{\text {th }}$ iteration are obtained by

$$
\begin{aligned}
& \Delta w_{(n) m+1}=\frac{1}{1+\alpha}\left[\alpha w_{(n) m-1}+\Delta w_{(n) m}\right] \\
& \Delta \varepsilon_{i j(n) m+1}^{\prime \prime}=\frac{1}{1+\alpha}\left[\alpha \Delta \varepsilon_{i j(n) m-1}^{\prime \prime}+\Delta \varepsilon_{i j(n) m}^{\prime \prime}\right]
\end{aligned}
$$

As $\alpha$ approaches zero or infinity, the new trial magnitudes of $\Delta w$ and $\Delta \varepsilon_{i j}^{\prime \prime}$ for the $(m+1)$ th iteration tends to equal the calculated and trial values of displacement and plastic strain components of the previous cycle, 
respectively. Hence for an a value between zero and infinity, intermediate values of $\Delta w$ and $\Delta \varepsilon_{i j}$ are obtained.

In general, two factors affecting the accuracy of the numerical solution are the degree of convergence tolerance and the mesh size. The accuracy of numerical solutions may be increased by utilizing a low convergence tolerance and a high number of grid points. This would result in an increase in the number of iterations for convergence to the final solution. There is, of course, a trade off between solution accuracy and computational efficiency which must be taken into account. In this study the accuracy of fixed boundary solutions was observed to be highly sensitive to the number of grid points utilized adjacent to the clamped boundary. For simply supported and free boundaries, however, far less sensitivity to the number of grid points was observed.

For elasto-plastic solutions, the magnitude of the applied lateral load increment $\Delta q$ has also been found to influence the stability and speed of convergence of the numerical procedure. Hence, it is preferable to use a constant small load increment when attempting elasto-plastic solutions. This is specially true for plates with other clamped edges.

In the example problems considered in this study, convergence to final solutions was achieved quite rapidly even when small tolerances and a rather large number of grid points were considered. The method of solution presented herein has been found to be computationally efficient and offers an alternate means of obtaining elasto-plastic solutions to circular and annular plate problems with moderately large deflections. 


\section{CHAPTER $V$}

\section{NUMERICAL RESULTS AND DISCUSSION}

A computer program based on the previously discussed method of solution was written. Solutions for two distinct classes of plates were obtained using this program:

1. Circular Plates

Elastic and elasto-plastic solutions of uniformly loaded simply supported and clamped circular plates undergoing large deflections have been obtained. The edges for both support conditions have been assumed immovable in the plane of the plate. Accordingly, the boundary conditions are given as:

$$
\begin{array}{ll}
\text { simply supported plates } & \text { o } r=a, w=M_{r}=u_{0}=0 \\
\text { clamped plates } & 0 r=a, w=\phi=u_{0}=0
\end{array}
$$

2. Annular Plates

Uniformly loaded annular plates with a free inner edge at $r=b$ and both simply supported and $\mathrm{clamped}$ outer edges were al so considered. In all cases, the ratio of the inner to outer radii was assumed to be 0.25 and the edges were considered immovable. Hence, the boundary conditions are:

$$
\begin{aligned}
\text { simply supported, free edges a } r & =a, \quad W=M_{r}=u_{0}=0 \\
& 0 r=b, \quad N_{r}=M_{r}=V_{r}=0
\end{aligned}
$$




$$
\begin{array}{ll}
\text { clamped, free edges } & \text { o } r=a, \quad w=\phi=u_{0}=0 \\
& 0 r=b, \quad N_{r}=M_{r}=v_{r}=0
\end{array}
$$

Where $V_{r}=$ Kirchhoff shear $=Q_{r}$ for an axisymmetric plate. The plate material is made of 2024-T351 aluminum alloy and is considered isotropic in the elastic and plastic regions. The following material properties and constants $\sigma_{0}$ and $n$ have been used in the numerical example problems:

$$
\begin{aligned}
& E=10 \times 10^{6} \mathrm{psi}(69 \mathrm{GPa}), \nu=0.3^{\star}, \sigma_{y}=32 \mathrm{ksi}(221 \mathrm{MPa}) \\
& \sigma_{0}=7.435 \times 10^{4} \mathrm{psi}(513 \mathrm{MPa}) \quad, n=3.08
\end{aligned}
$$

Initially, a suitable mesh size was selected in the nonlinear elastic analysis of circular and annular plates. The results were then compared with the available solutions in order to establish the accuracy of the solution procedure and to asses the computational efficiency of the method. The nonlinear elastic solutions for circular and annular plates are shown in Figs. 7 through 16 . The corresponding available solutions are also included for comparison.

As a next step, the problem was extended beyond the elastic limit by considering the elastic-plastic behavior of circular and annular plates undergoing moderately large deflections. The plate is first loaded

* A value of $v=0.25$ has been used in the elastic simply supported circular plate example problem in order to facilitate comparison with the available solutions. 
elastically until the effective stress of the extreme fiber at the center or the inner edge of the plate reaches the yield stress. Beyond the elastic limit, the incremental plasticity theory together with the iteration technique, discussed earlier, are used to obtain solutions to the example problems. The results of elastic-plastic plates are shown in Figs. 17 through 50. The nonlinear elastic solutions are also included for the purpose of comparison. The elastic-plastic analyses reported by other investigators have largely been confined to elastic-perfectly plastic material wherein the effect of strain-hardening has been neglected. Furthermore, the majority of the studies found in the open literature have employed the deformation theory of plasticity. The plastic strains can not in general be independent of the loading path and deformation theories can not generally be correct. Accordingly, no quantitative comparison of the present solutions $c$ an be made with the available solutions. However, it is possible to compare, on a qualitative basis, the elastic-plastic solutions of this study with the existing solutions of plates made of elastic-perfectly plastic materials which are based on an incremental plasticity theory.

A general discussion of the nonlinear elastic and elasto-plastic results obtained for the circular and annular plates considered in this study are presented herein. All results are presented in nondimensional form in order to facilitate comparison with those of other investigators when applicable. 
LARGE DEFLECTION, ELASTIC RESULTS

The nonlinear elastic results for both uniformly loaded simply supported and clamped circular plates are shown in Figs. 7 through 10. Fig. 7 shows the variation of the center deflection with load for a simply supported circular plate, while those corresponding to the extreme fiber radial stress at the center and the radial membrane stress at the edge of the $\mathrm{plate}$ are given in Fig. 8. The results of the present method are seen to agree very well with those of Nath, et al. (27) and Alwar and Nath (6).

The results for a $\mathrm{clamped}$ circular plate are presented in Figs. 9 and 10. The center deflection of the clamped circular plate, given in Fig. 9, was calculated and found to compare very well with the results of Nath, et al. (27) and Alwar and Nath (6). Fig. 10 shows the variation of the radial membrane and bending stresses with center deflection at the edge and the center of the plate. The comparison of these results with the existing solutions is also shown.

It is important to note that the solutions for both simply supported and clamped circular plates are in good agreement with the available solutions. The present solutions are, however, closer to those given in reference (27) for the range of the load considered. Moreover, an extremely favorable comparison with the series solution of $\mathrm{clamped} p l$ ates given by Way (5) is al so noted. Although not shown in the figures, as a further check on the accuracy of these solutions, the present results have been compared with dynamic relaxation solutions of Turvey (16) and found to be in close agreement. 
The nonlinear elastic solutions for both uniformly loaded simply supported and clamped annular plates are shown in Figs. 11 through 16. The load-deflection curves at the inner edge of the plate for both types of supports are given in Figs. 11 and 14, while the relations between the radial and tangential bending stresses at the inner and outer edges of the plate and the applied lateral pressure are shown in Figs. 12 and 15. Figs. 13 and 16 represent the radial and tangential membrane stresses at the plate boundaries as computed by the proposed method. The solutions for simply supported and clamped annular plates show excellent agreement with the results of Dumir, et al. (30) and Turvey (16).

LARGE DEFLECTION, ELASTIC-PLASTIC RESULTS

The results of the elastic-plastic analys is for simply supported and clamped circular plates are shown in Figs. 17 through 32 . The corresponding nonlinear elastic solutions are also included in the figures for comparison. Fig. 17 shows the variation of the deflection at the center with the nondimensional load $q(a / h)^{4} / E$ for a simply supported circular plate. The load deflection curve of the classical theory is also shown in the figure. This plot clearly indicates the effects of membrane forces and the plastic strain on the behavior of the plate. The displacement at the center of the plate is noted to increase by the plastic strain. The results for the radial and tangential membrane stresses at the center and the edge of the plate are plotted in Fig. 18. Fig. 19 gives the 
variation of the radial and tangential stresses with load at the center and edge of the plate. The results for stresses indicate that while the membrane stresses are only slightly affected by plastic strain, the corresponding maximum fiber stresses at the center are considerable relieved by the inelastic action, resulting in additional load carrying capacity for the plate and, of course, make better use of the material, an important consideration in aerospace applications. The distribution of the effective plastic strain at a nondimensional load of 7.15 is presented in Fig. 21. The results indicate that the center of the plate at the lower surface yields initially. As the load is increased, the plastic range propagates toward the top and the edges. Before the plastic deformation completely reaches the edges, however, the upper surface of the edge starts yielding and the plastic deformation in turn propagates toward the center of the plate. Figs. $21,22,23$, and 24 plot, respectively, the radial profiles of lateral deflection, extreme radial stress and radial membrane stress, extreme tangential stress and the corresponding membrane stress, and sectional moments $M_{r}$ and $M_{t}$ for the nonlinear elastic and elasto-plastic solutions of the simply supported circular plate.

Next, the elastic-plastic behavior of a clamped circular plate under a uniformly distributed load is analyzed. The results are presented in Figs. 25 through 32 . The plot of the lateral displacement of the center, given in Fig. 25, shows that, unlike a simply supported plate, when the load parameter increasess beyond a certain value, the displacement corresponding to the elastic-plastic large deflection solution exceeds slightly that of the linear elastic case. This behavior is attributed to 
the fact that the increase in displacement due to the effect of plastic strain exceeds the reduction in the value of the deflection resulting from the second order effects of lateral displacement. This finding is consistent with the results of Crose and Ang (56) and Turvey and Lim (43). The plots for the radial and tangential membrane stresses at the center and the edge of the plate are given by Fig. 26; it is noted that the membrane stresses are slightly increased by the inelastic action. Fig. 27 shows the variation of the radial and tangential stresses at the center and the edge of the plate. Again, the maximum stresses are observed to be considerably reduced in the elastic-plastic case. The distribution of the effective plastic strain in the clamped circular plate is shown in Fig. 28 . As seen from the figure, yielding starts initially at the top surface of the edge. At a slightly higher load, the bottom surface of that boundary also yields and the plastic strain propagates toward the center of the plate. However, before it reaches the center, the lower surface of the center yields and the plastic strain in turn propagates toward the edge. Fig. 29 shows the radial profile of lateral deflections of the plate at a nondimensional load of 5.96. Figs. 30 and 31 show the distribution of the maximum radial and tangential stresses together with the corresponding membrane stresses for the nonlinear elastic and elasto-plastic solutions. Additionally, the variation of the nondimensional moments $M_{r}$ and $M_{t}$ along a radial line are given in Fig. 32 .

The results of the elastic-plastic analys is of simply supported and clamped annular plates are shown in Figs. 33 through 50 . The corresponding nonlinear elastic solutions are also included. Figs. 33 and 42 show, 
respectively, the variation of the deflection at the inner edge of the simply supported and clamped plates with the nondimensional load. The load deflection curves of the classical linear theory are al so shown in these figures. The radial and tangential membrane stresses at the inner and outer boundaries of both plates are plotted in Figs. 34,35 , and 43,44 while the result for the extreme fiber stresses are shown in figs. 36 and 45. The distribution of the effective plastic strain, shown in Figs. 37 and 46 , clearly indicate the regions of the plates that have yielded.

Figs. 38 and 47 plot the radial profiles of lateral deflections at the nondimensional loads of 3.58 and 5.96 for simply supported and clamped annular plates, respectively. Additionally, the distributions of the maximum radial and tangential stresses together with the corresponding membrane stresses and sectional moments $M_{r}$ and $M_{t}$ for the elastic-plastic and nonlinear elastic solutions are given in Figs. 39 to 41 and 48 to 50 .

The interpretation of the results for the simply supported and clamped annular plates is similar to that of corresponding circular plates and, therefore, will not be repeated. 


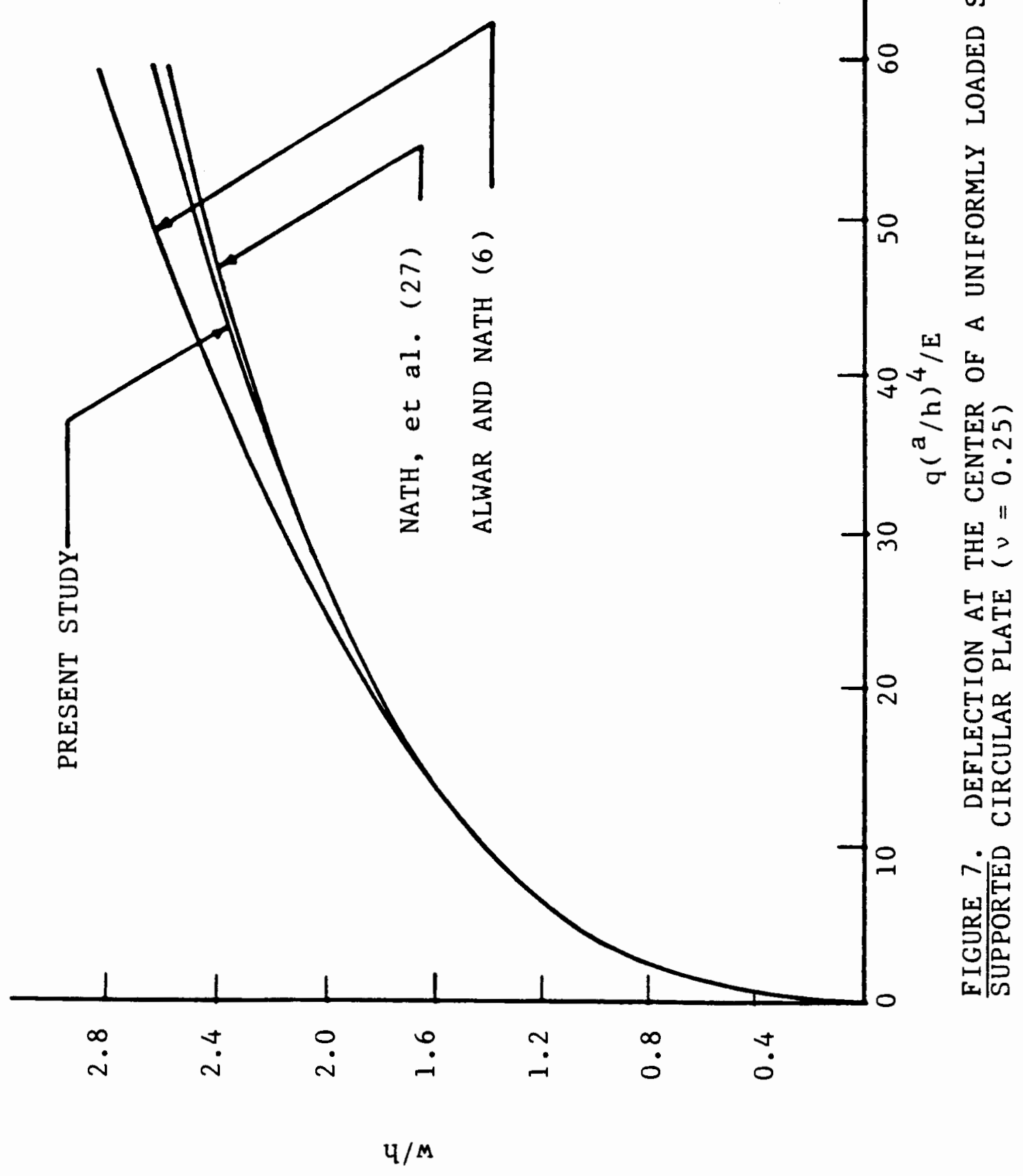




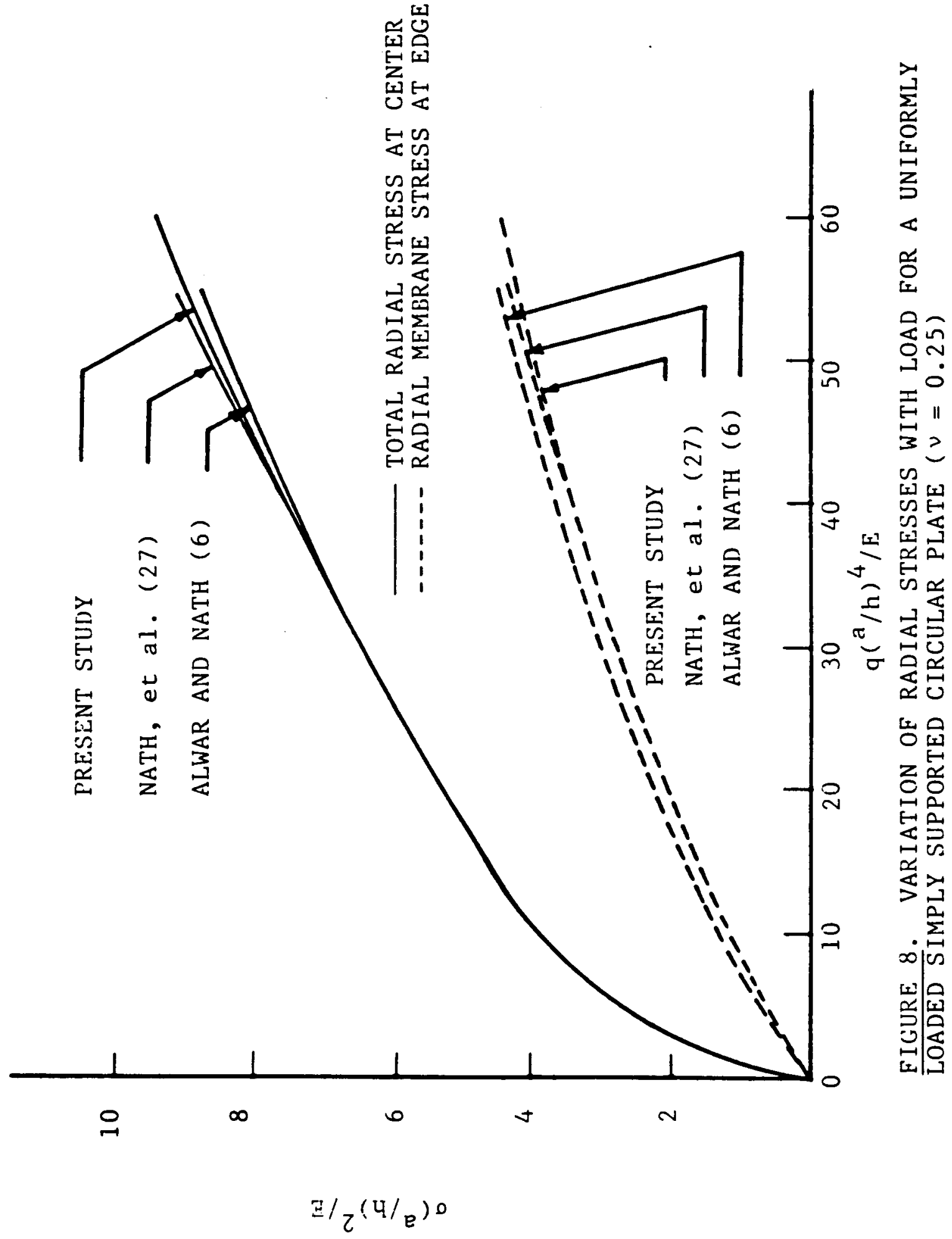




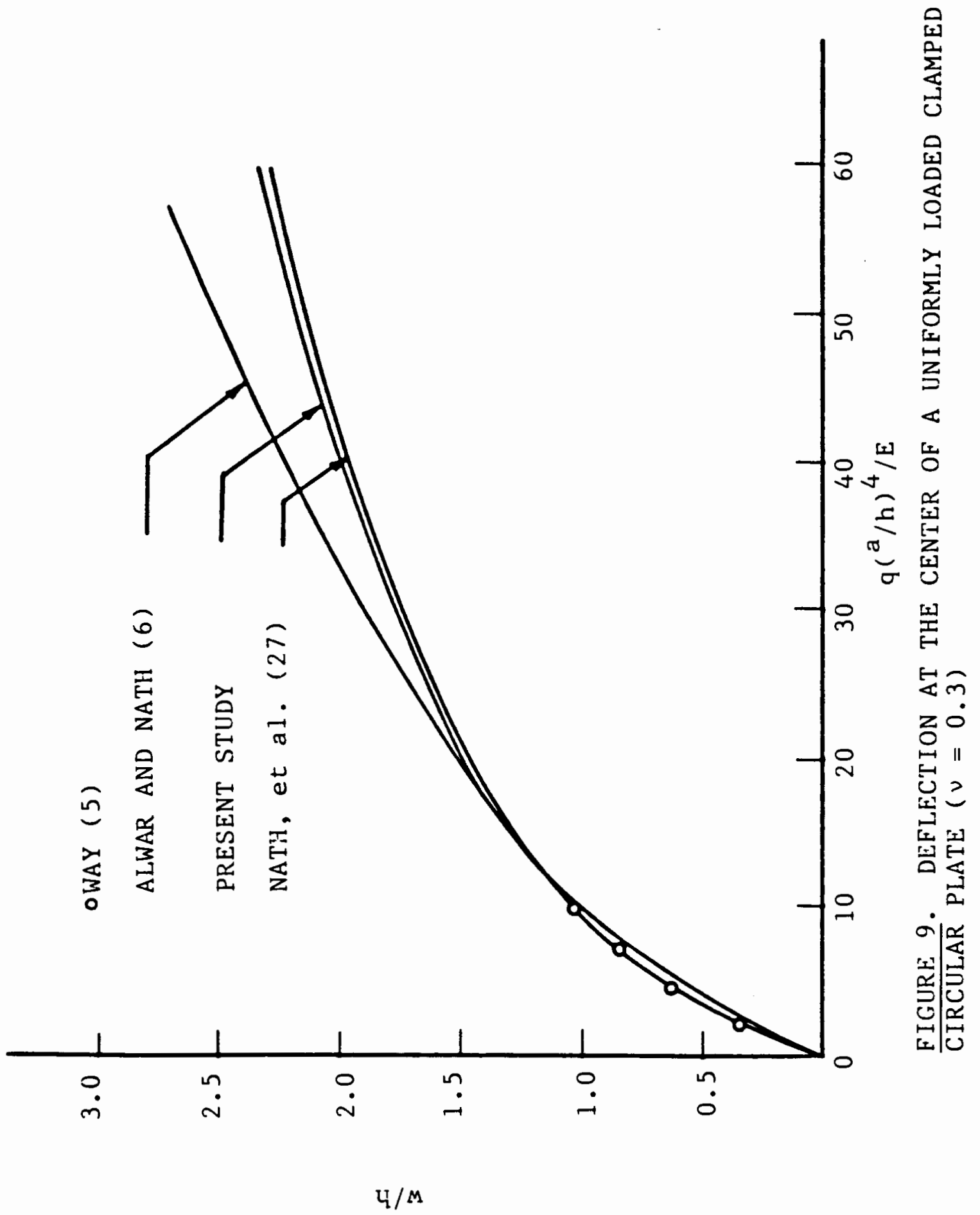




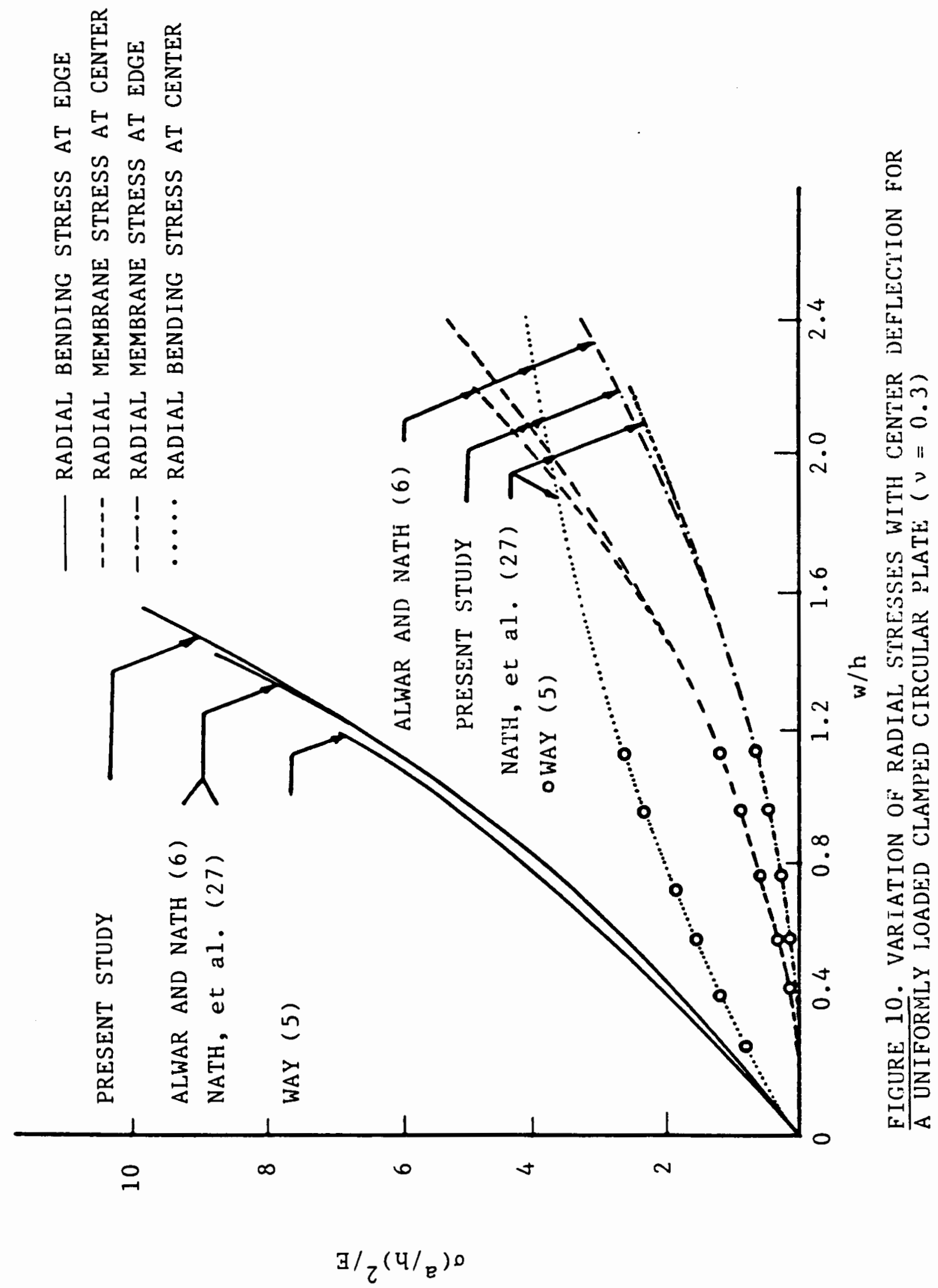



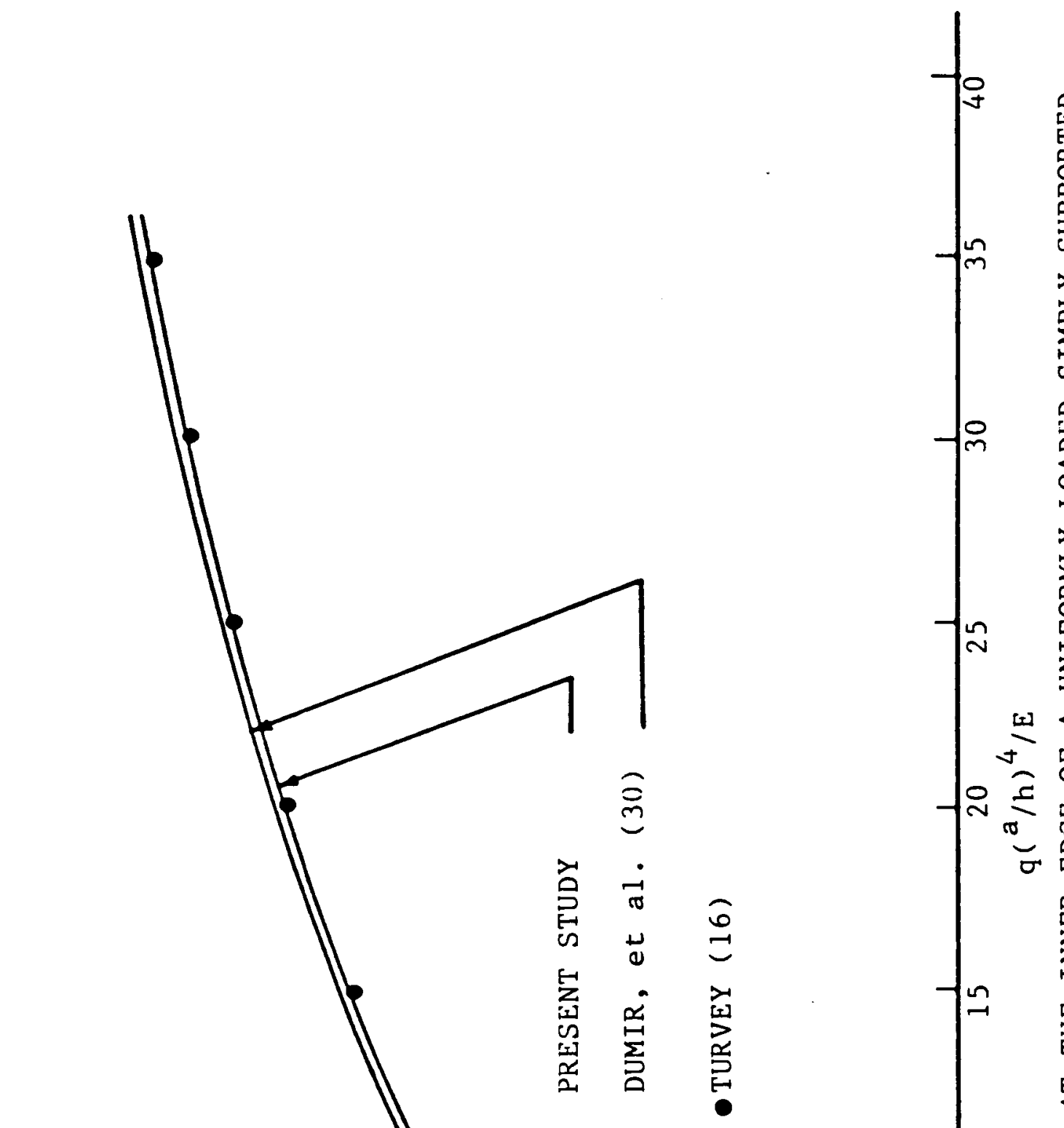

일

竞

- 0 용

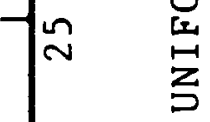

다 4

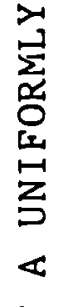

농

-

獣领

ZN

$z$ :

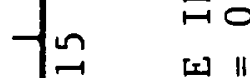

됱 입

晷。

zं?

붕

包 I

걱 ?

点一

됨 도

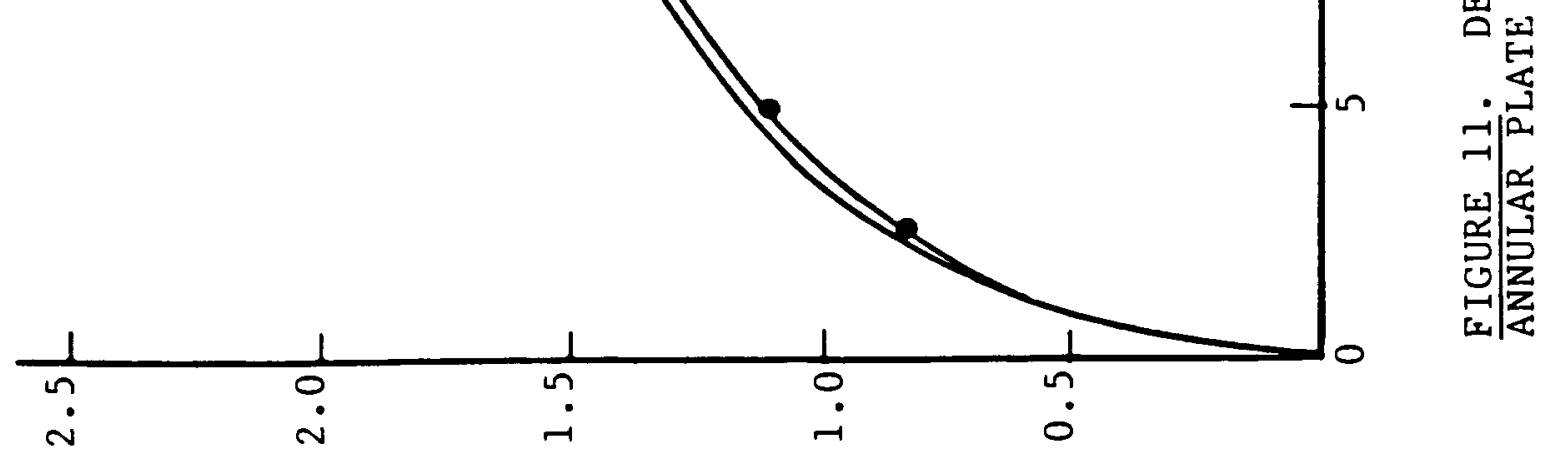

บ/M 


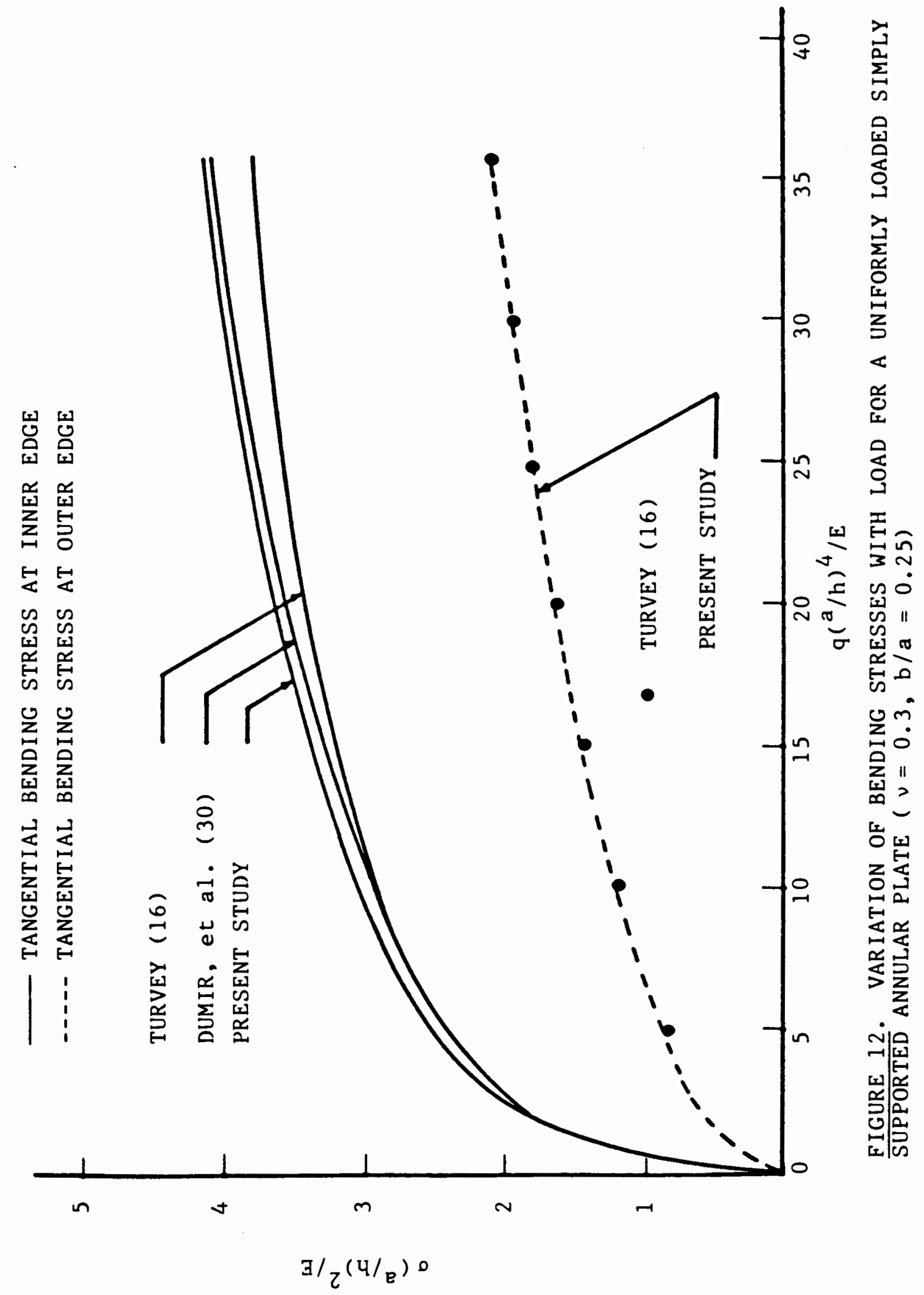




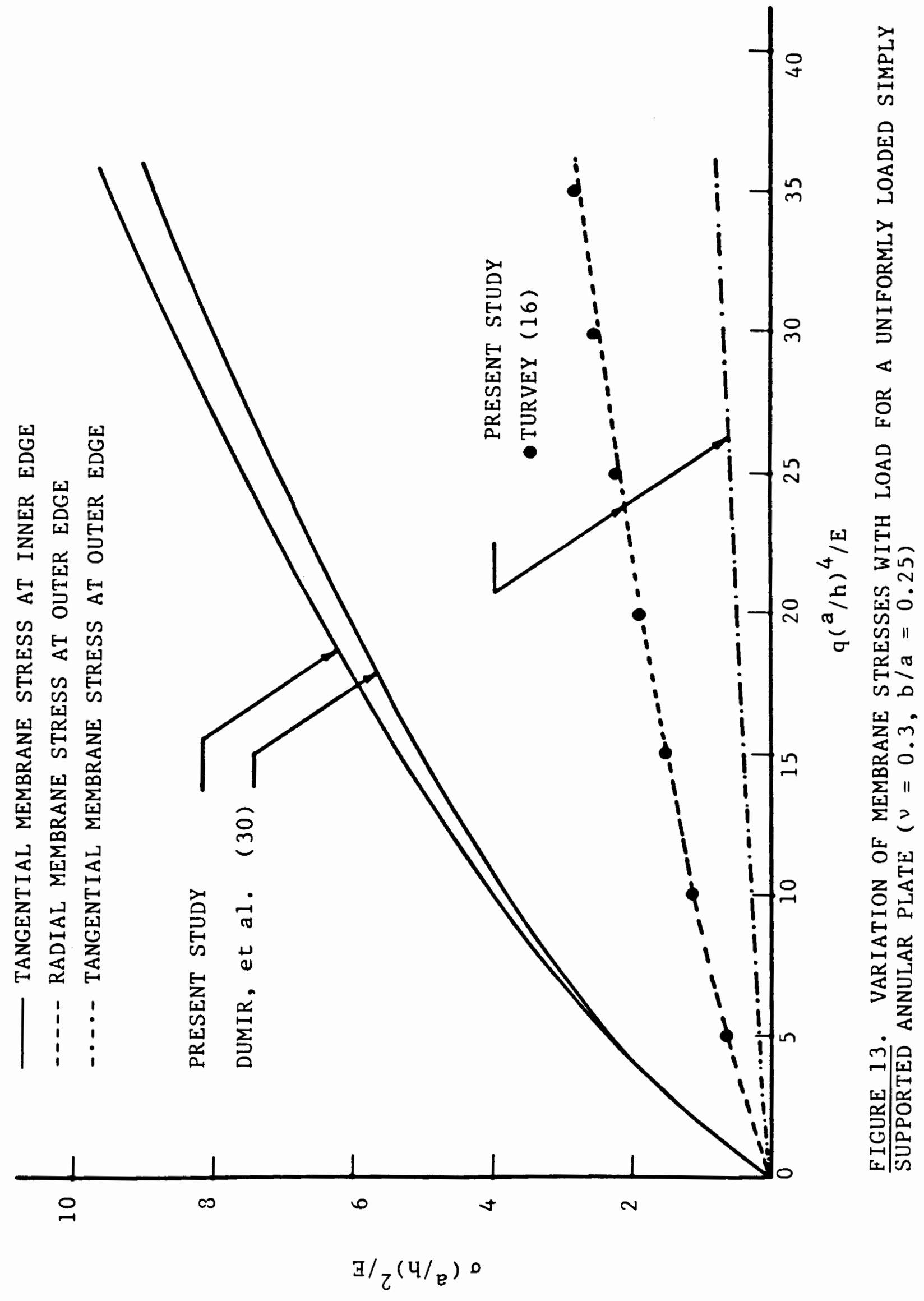




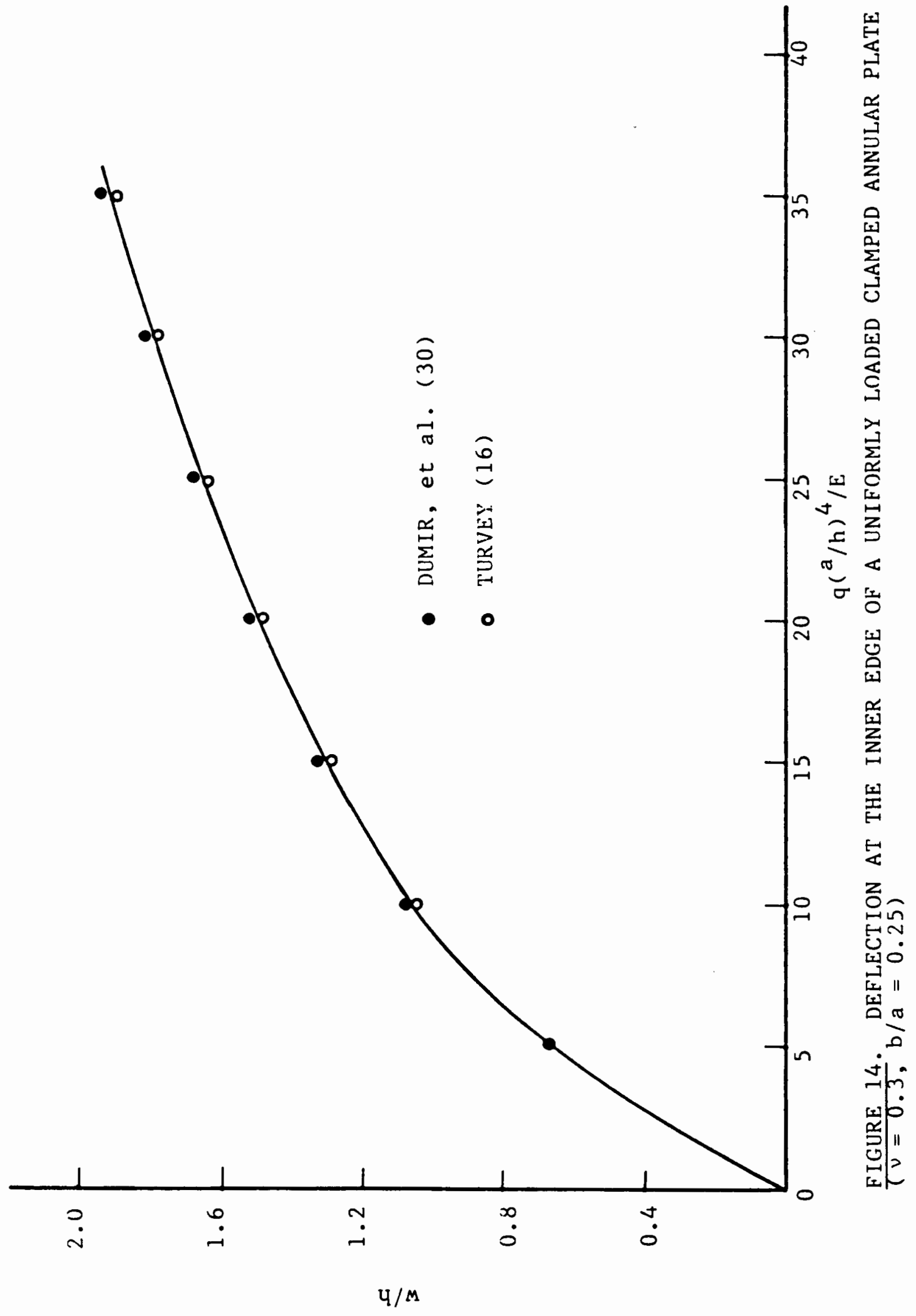




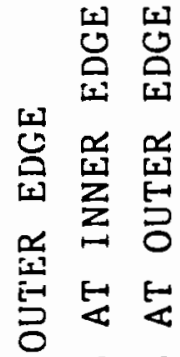

E i u

$<$ ज 단

\& है

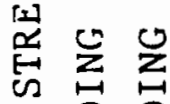

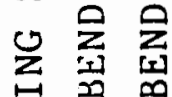

도

$\infty$

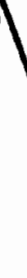

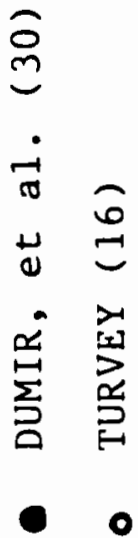

$\infty_{\infty} \quad \begin{aligned} & 1 \\ & \vdots \\ & \vdots\end{aligned}$

fo

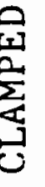

我导导

占皆

䍐心

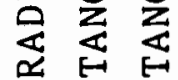

$\mid \begin{aligned} & \vdots \\ & \vdots \\ & \vdots\end{aligned}$ 
먼

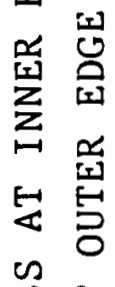

䄈

趈

牙

璐

空塄

安览

$\boxminus \Sigma$

$\sum_{i \rightarrow 3}$

怘完

1

ô

10

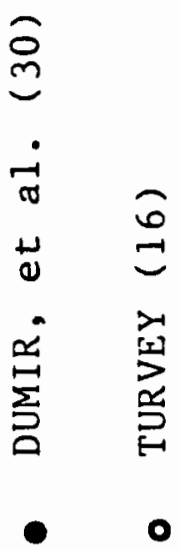

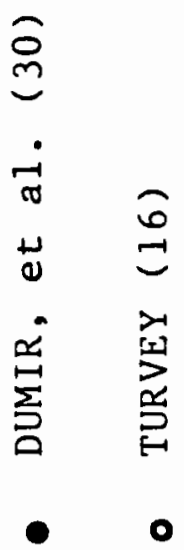

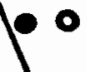

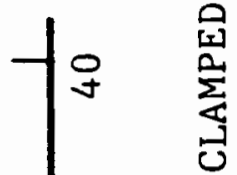

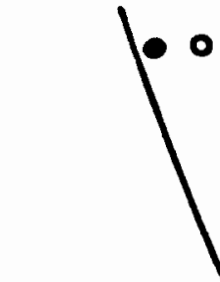

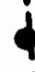

m

뇡

章

$\varangle$

范
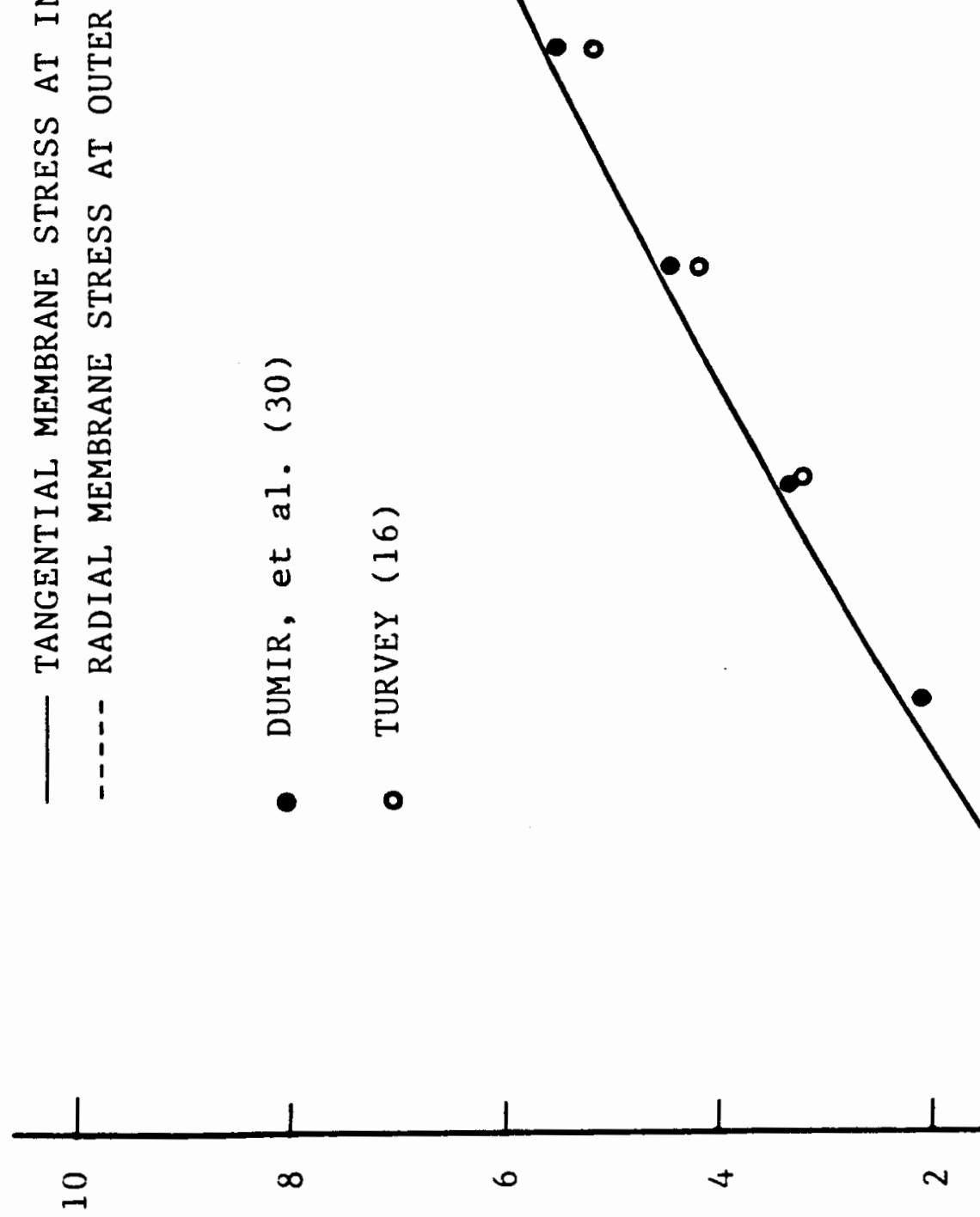

$\checkmark$

$\left.\exists / \gamma^{(4 / e}\right) 0$ 


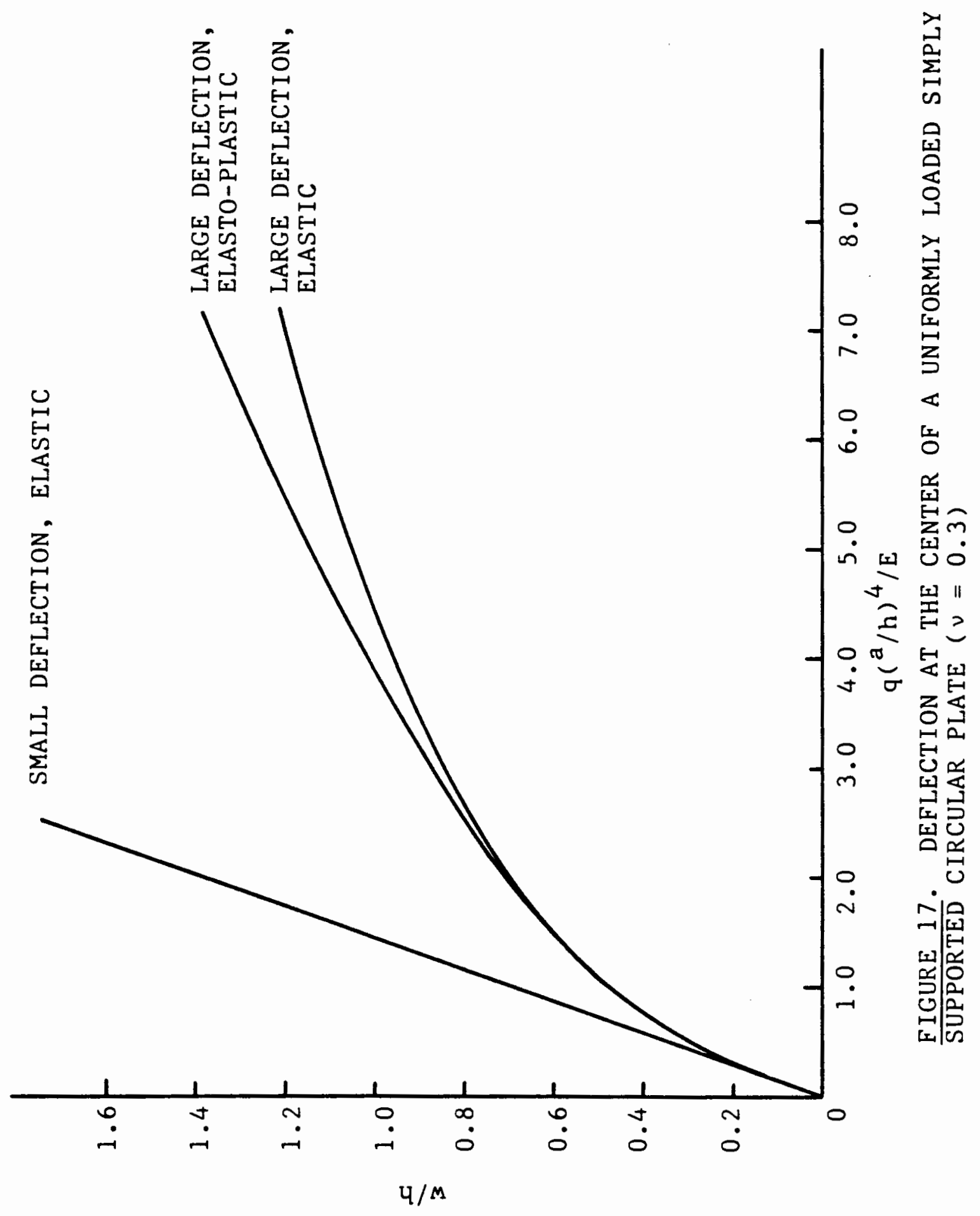




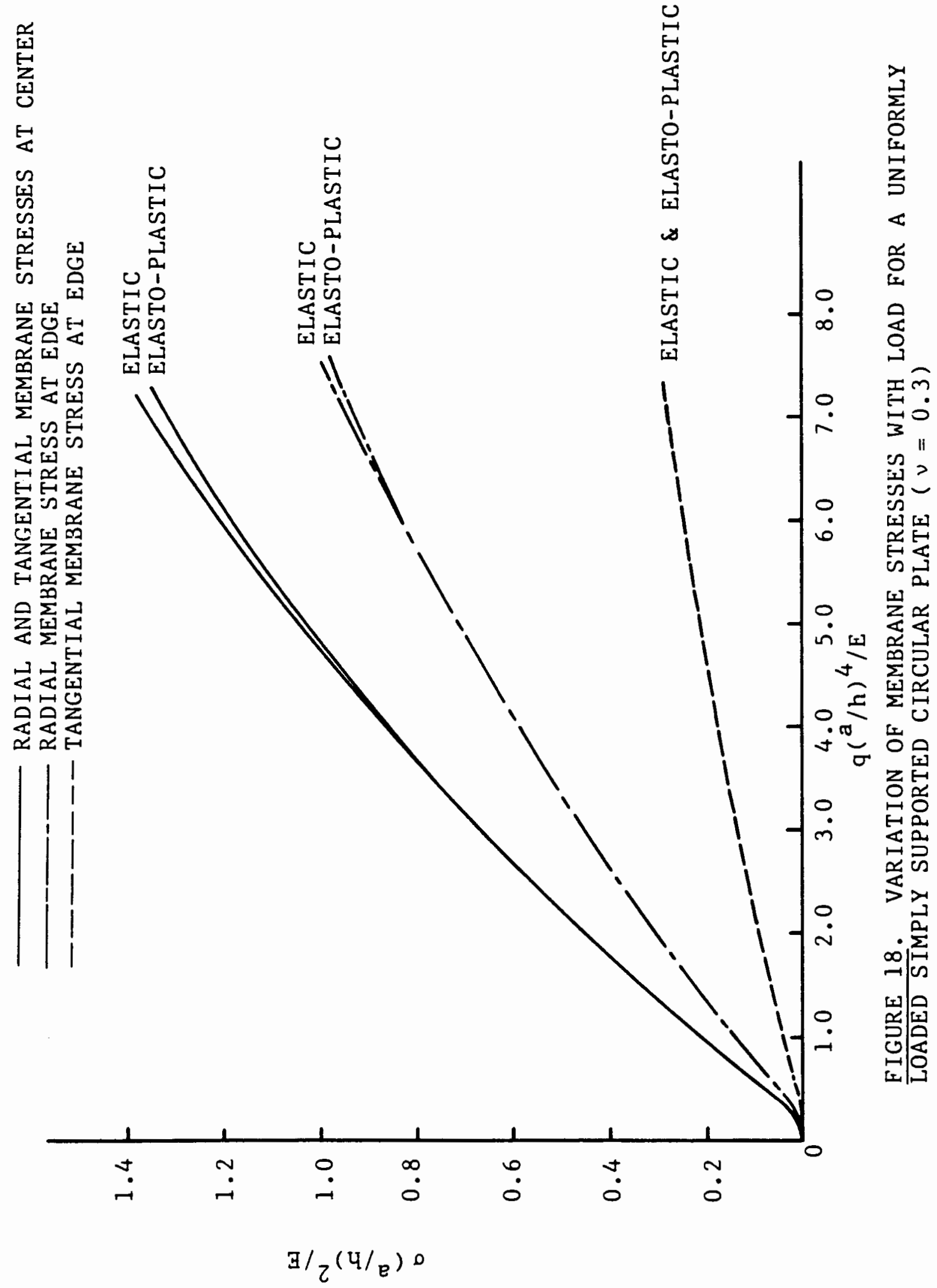


乩

它

넌

동

的可

똔

号占

.

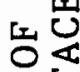

足

的

๑

됨면

告要

थ

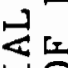

$\mapsto 0$

皆的

[ᄃㄴ

던

$\sum \frac{\alpha}{E}$

룬

4

늘

$\varangle$ 되

占心

$\varangle<$

品

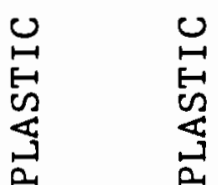

尝

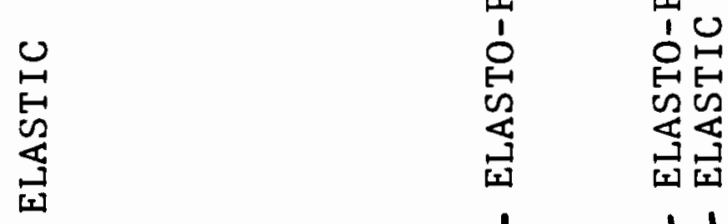

$<$

$\infty$

옹

近
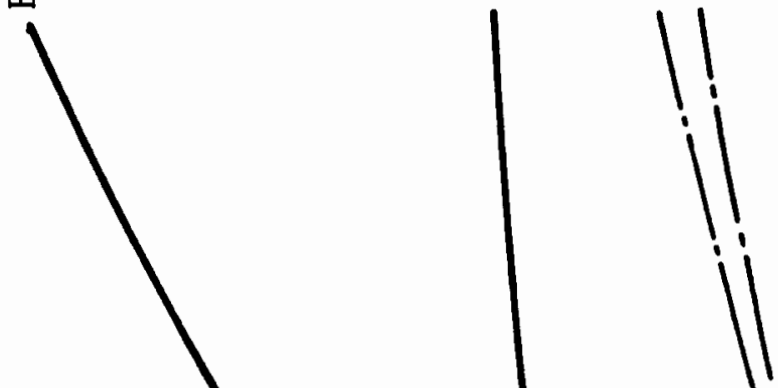

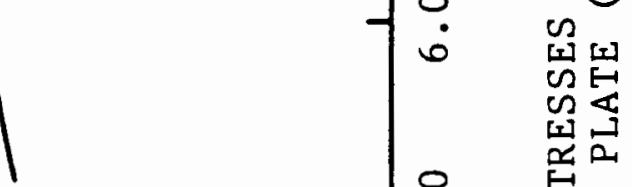

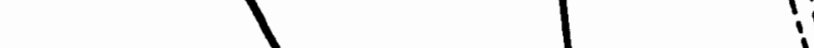




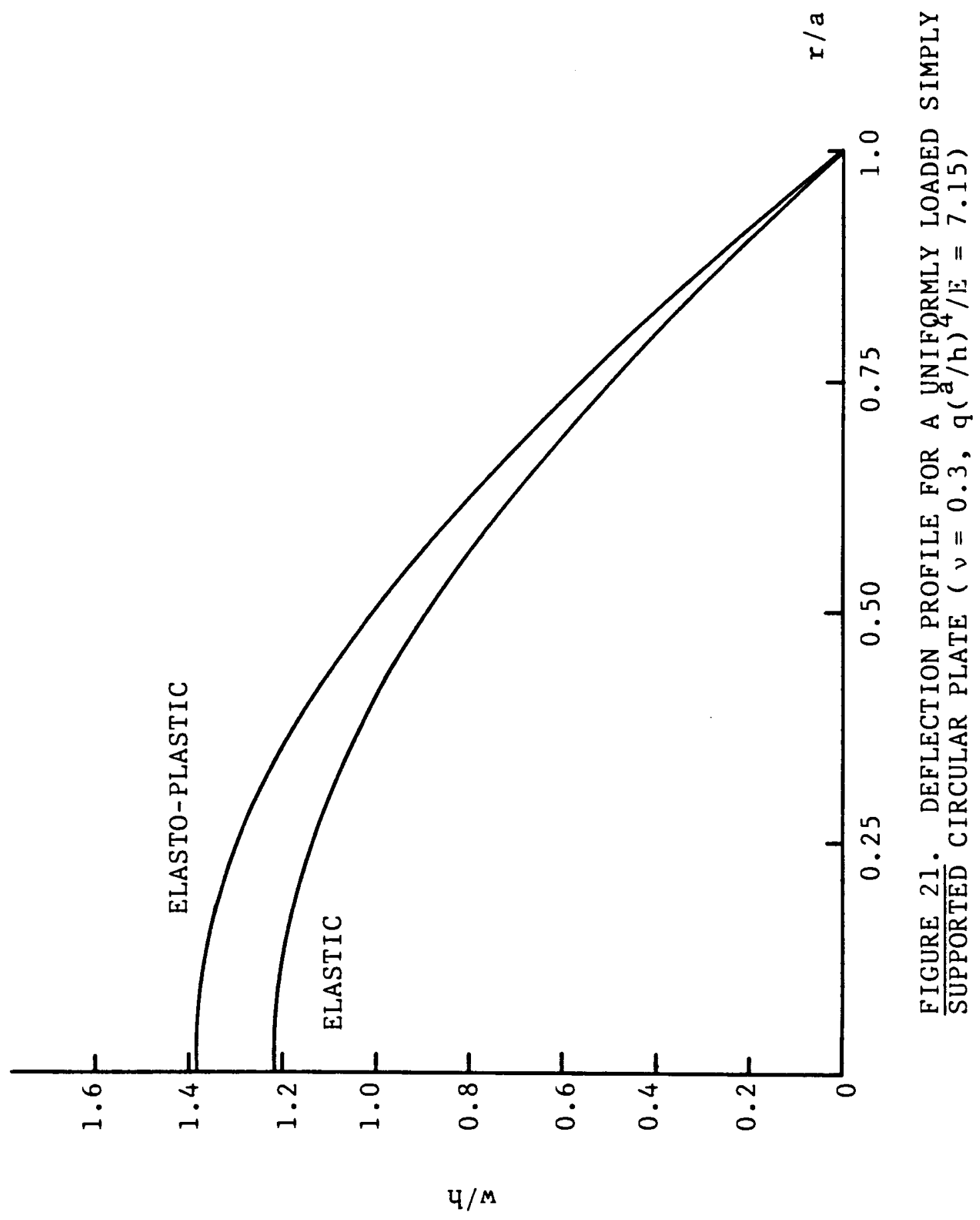




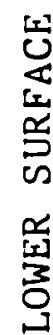

出战

थ只

wis

品

is 돌

完

닝노

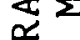

完

突它

$\mid$
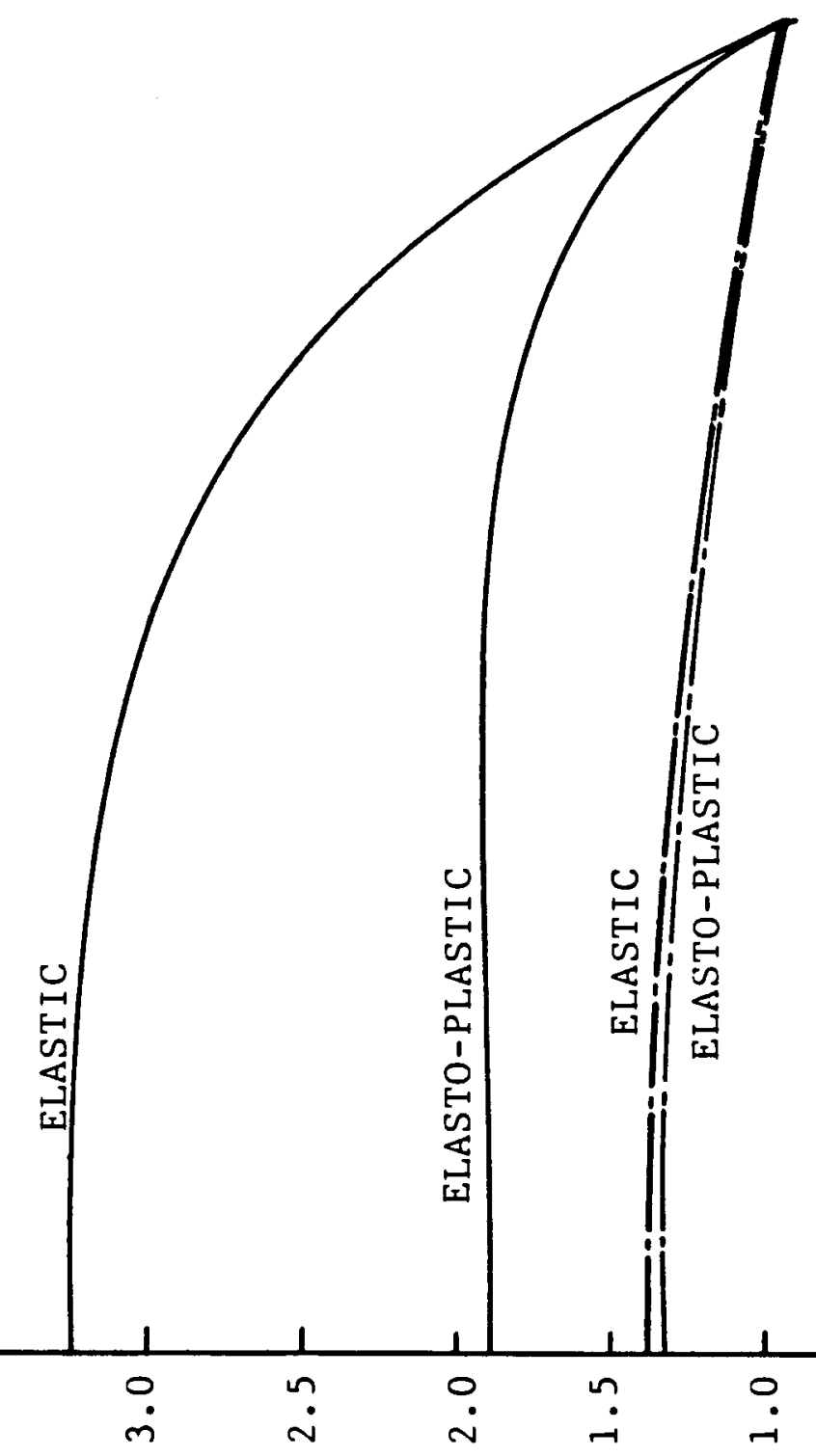

$\frac{\pi}{8}$

点

4

○

도

$\eta \rightarrow$

오ำ

술

"I

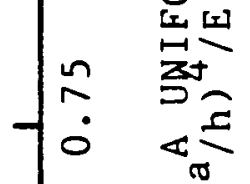

$\pi$

옹 б

豆

동

율 옴

० w

되원

䆛吕

这号

$\checkmark 1$

出

里!

四骂

这苞

v 0

N)

N

뇡

氙坛

$\exists / Z(4 / e) 0$ 


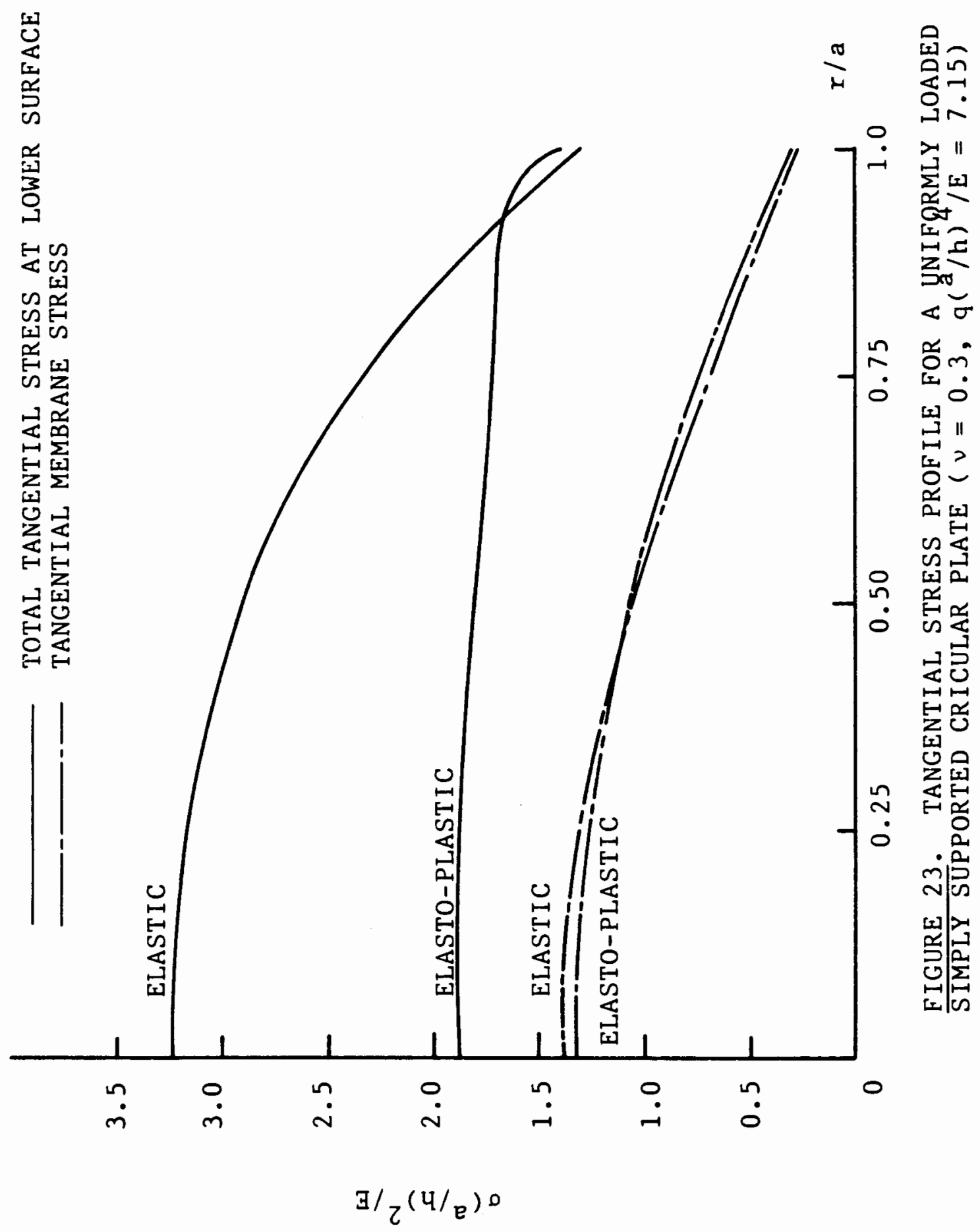




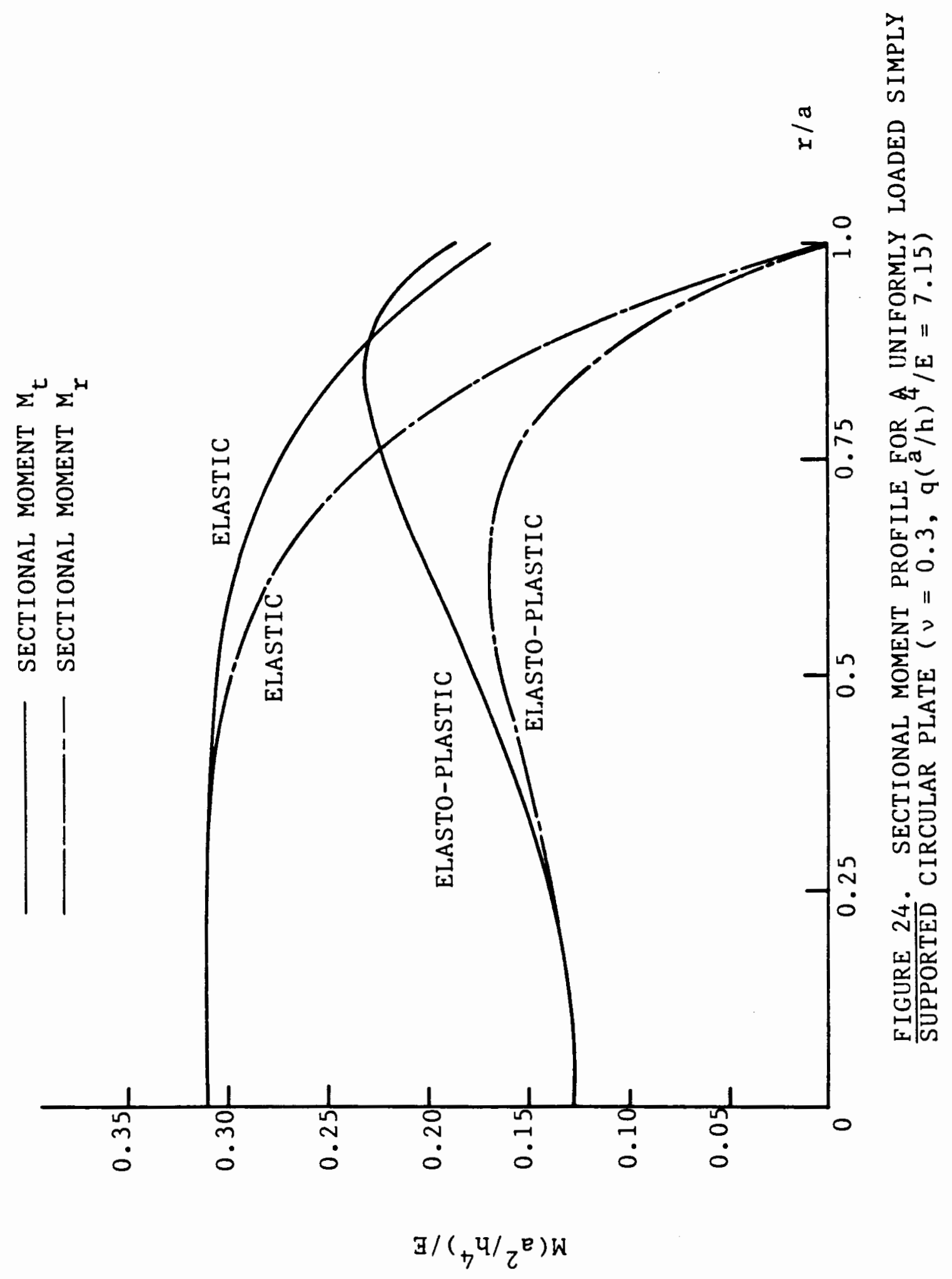




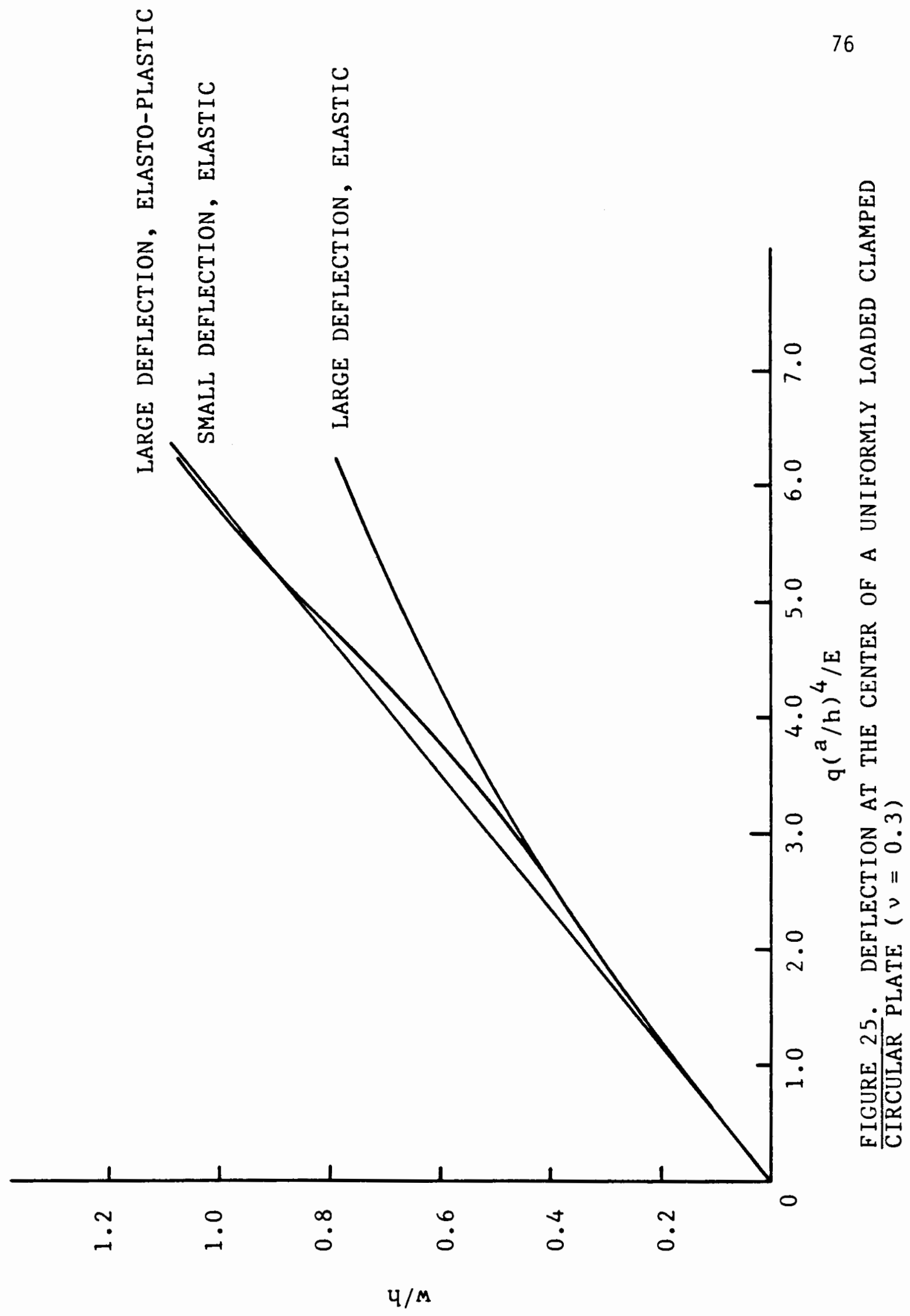


空

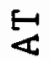

告

된됩

近四

品

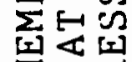

定

丞约

$\varangle$ 됭

由㟔

$\sum_{\text {에 }}$

됭

乙织

出本定

일 뇐

正焉

$\rightarrow \cdots \stackrel{\mathrm{E}}{\mathrm{Z}}$

攺

본

这安

$\alpha \propto$

$\mid \begin{aligned} & 1 \\ & 1 \\ & 1 \\ & 1 \\ & 1\end{aligned}$

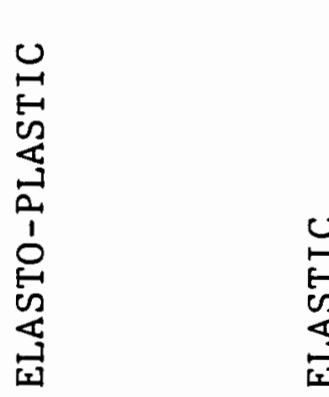


䍃

E

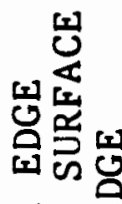

또되 미

穹帘皆

世논

$40<$

뫼 $\Omega$

此穴

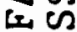

$\alpha$ 되 오

ن

$\alpha$ थ

牙造这

田员

点心

4 式

○四

$\Omega \geq$

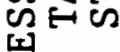

品是是

굴

$<4$

은

台乩

$|1|$

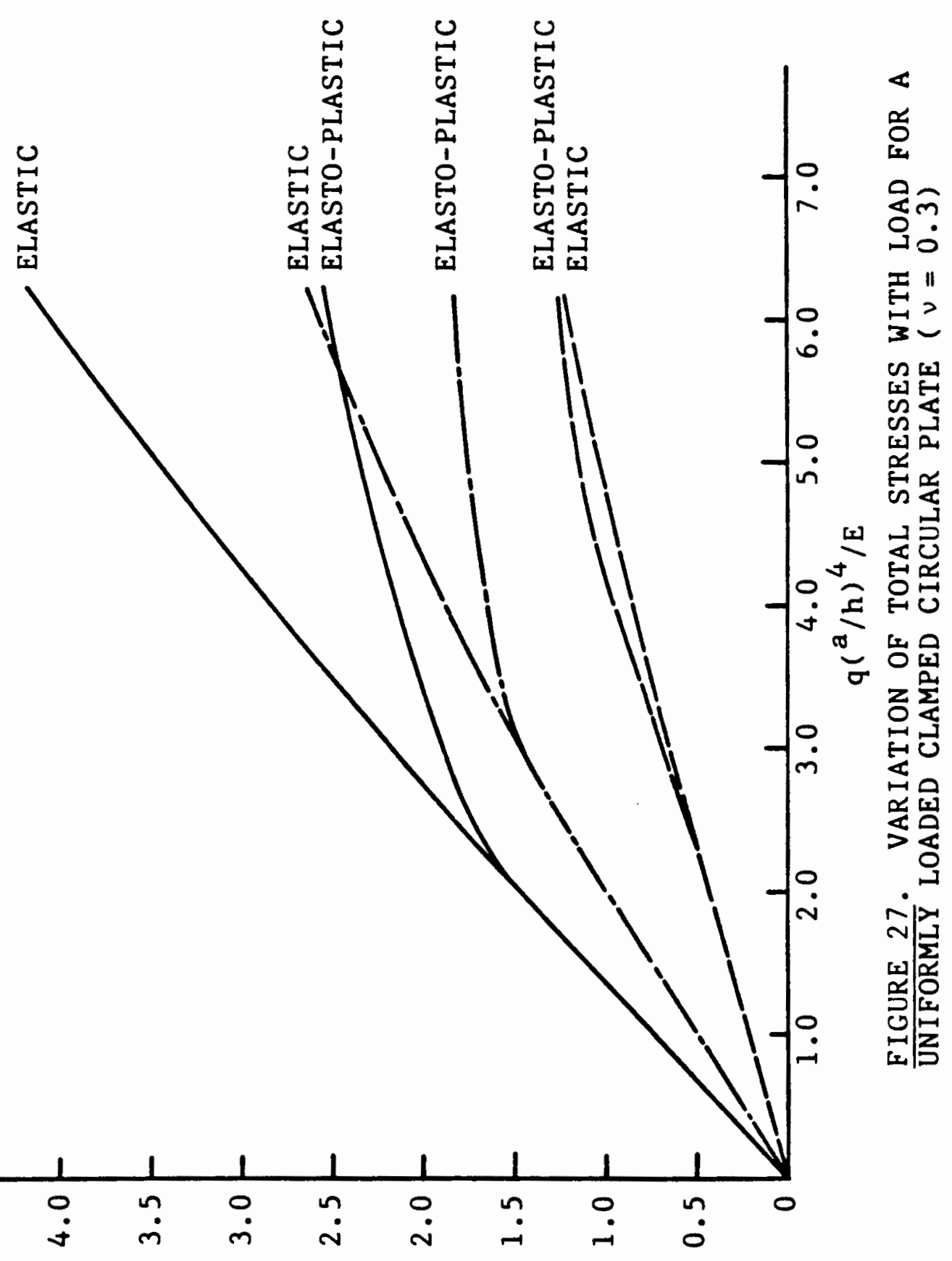

$3 / 7(4 / e) 0$ 


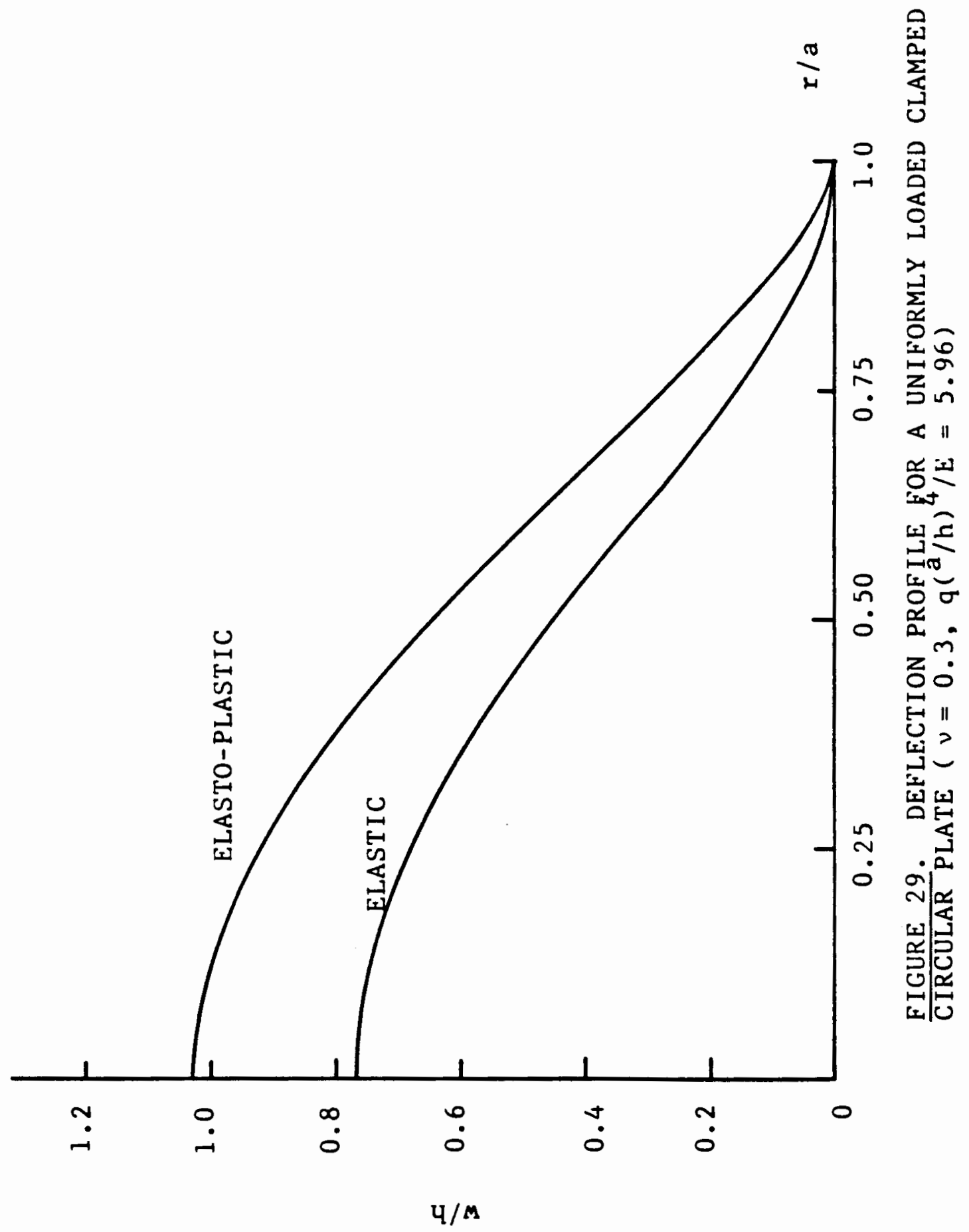


뇐

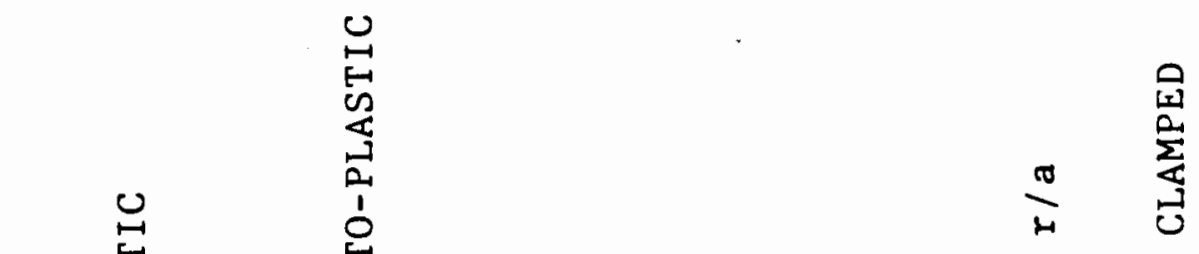

品

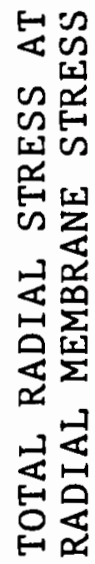

1
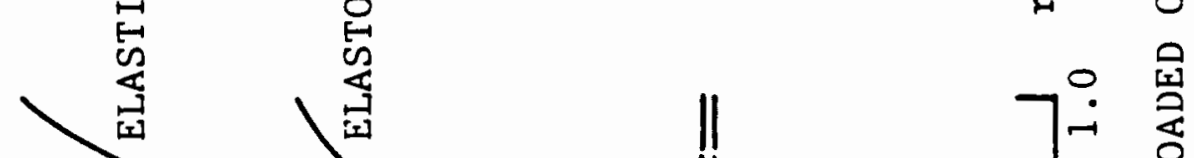

1

$\checkmark$

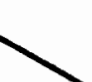




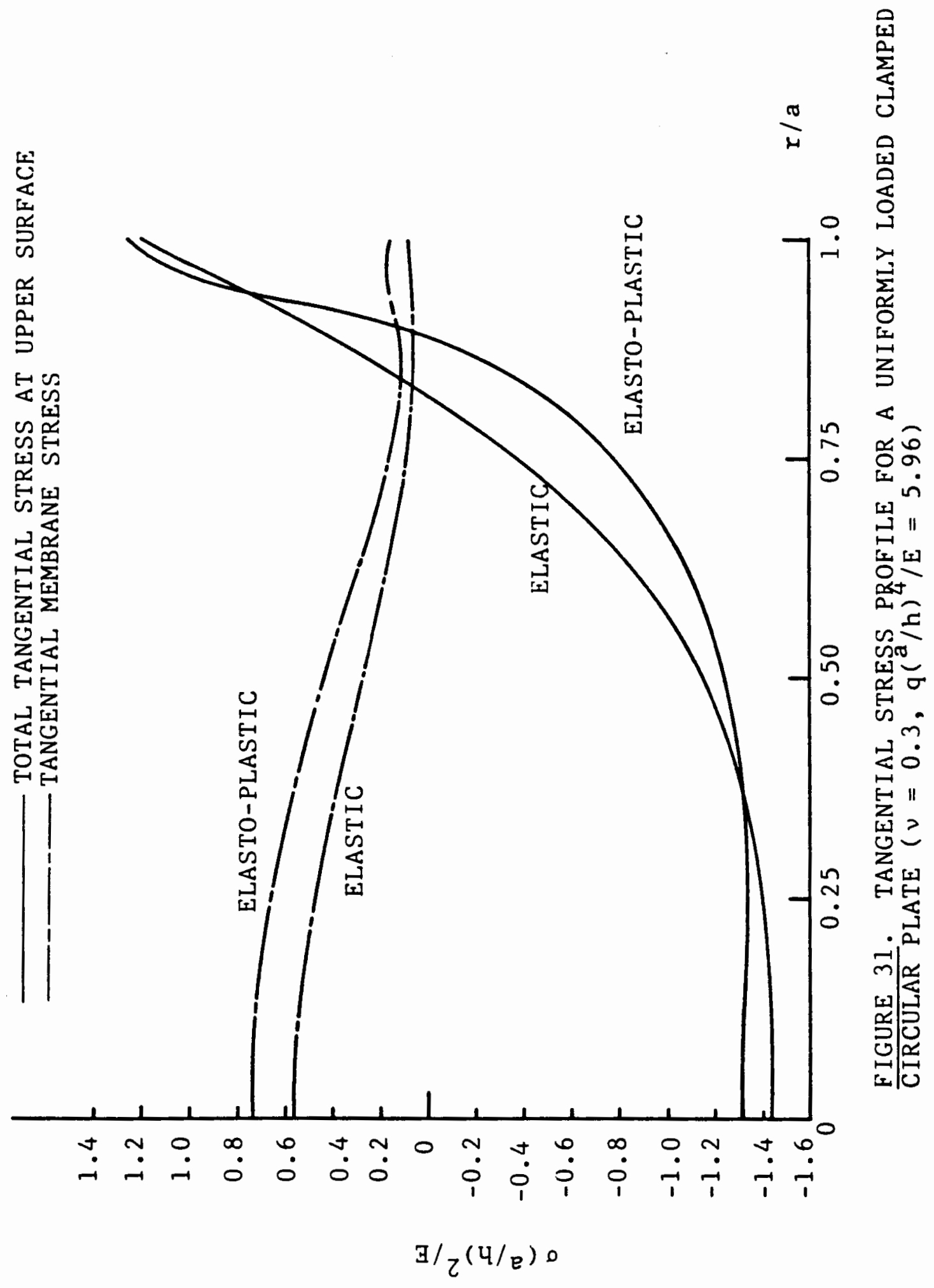




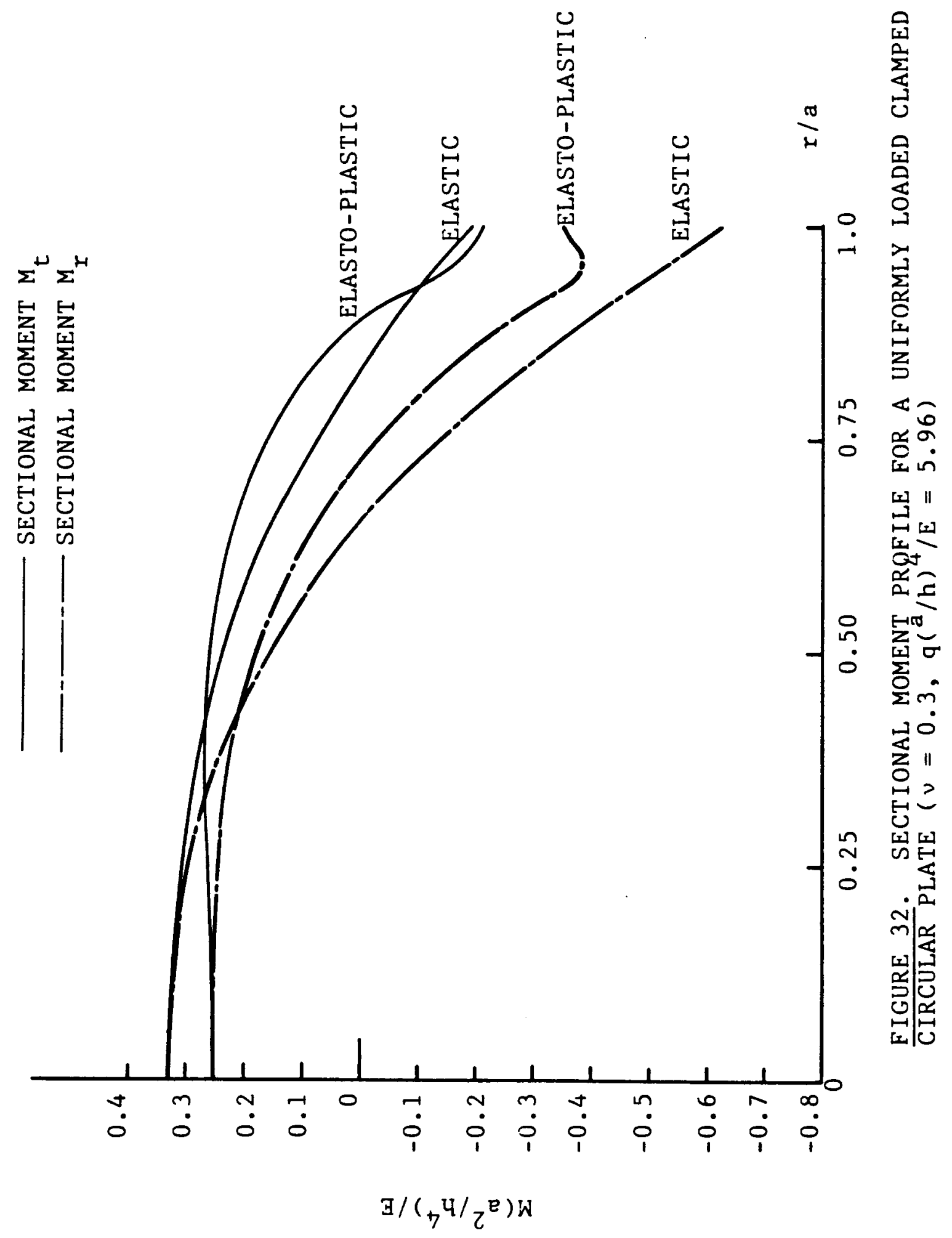




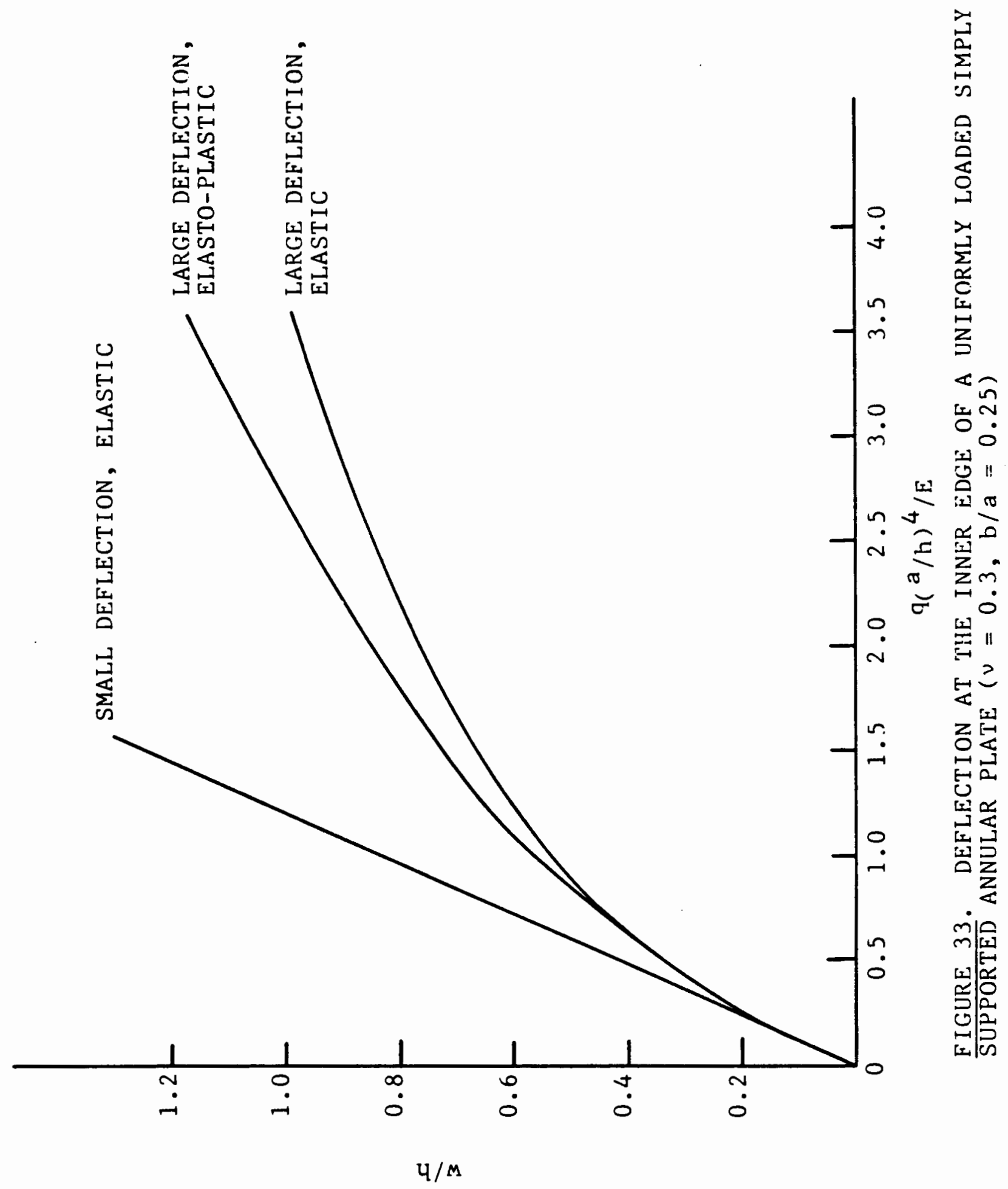




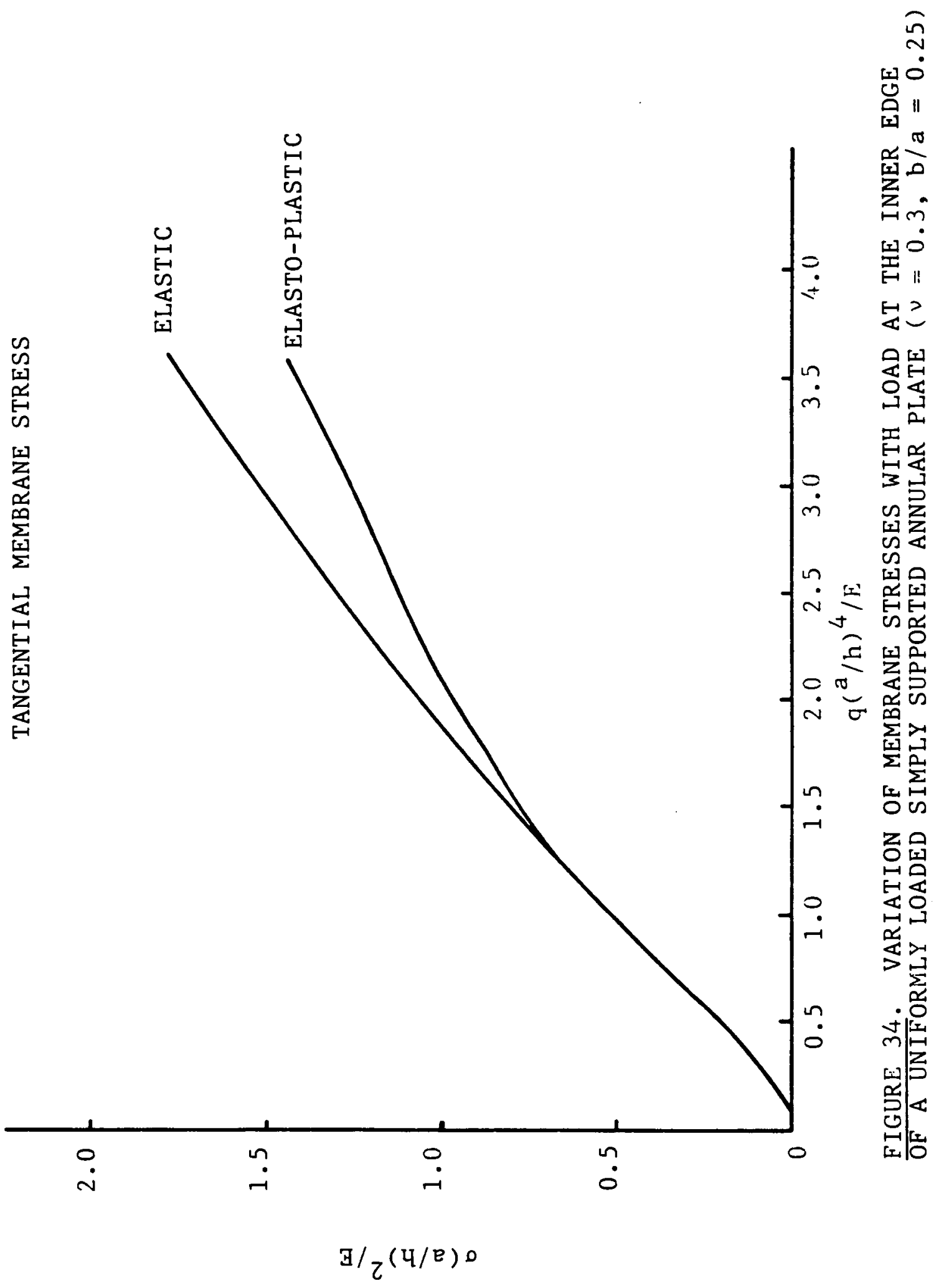




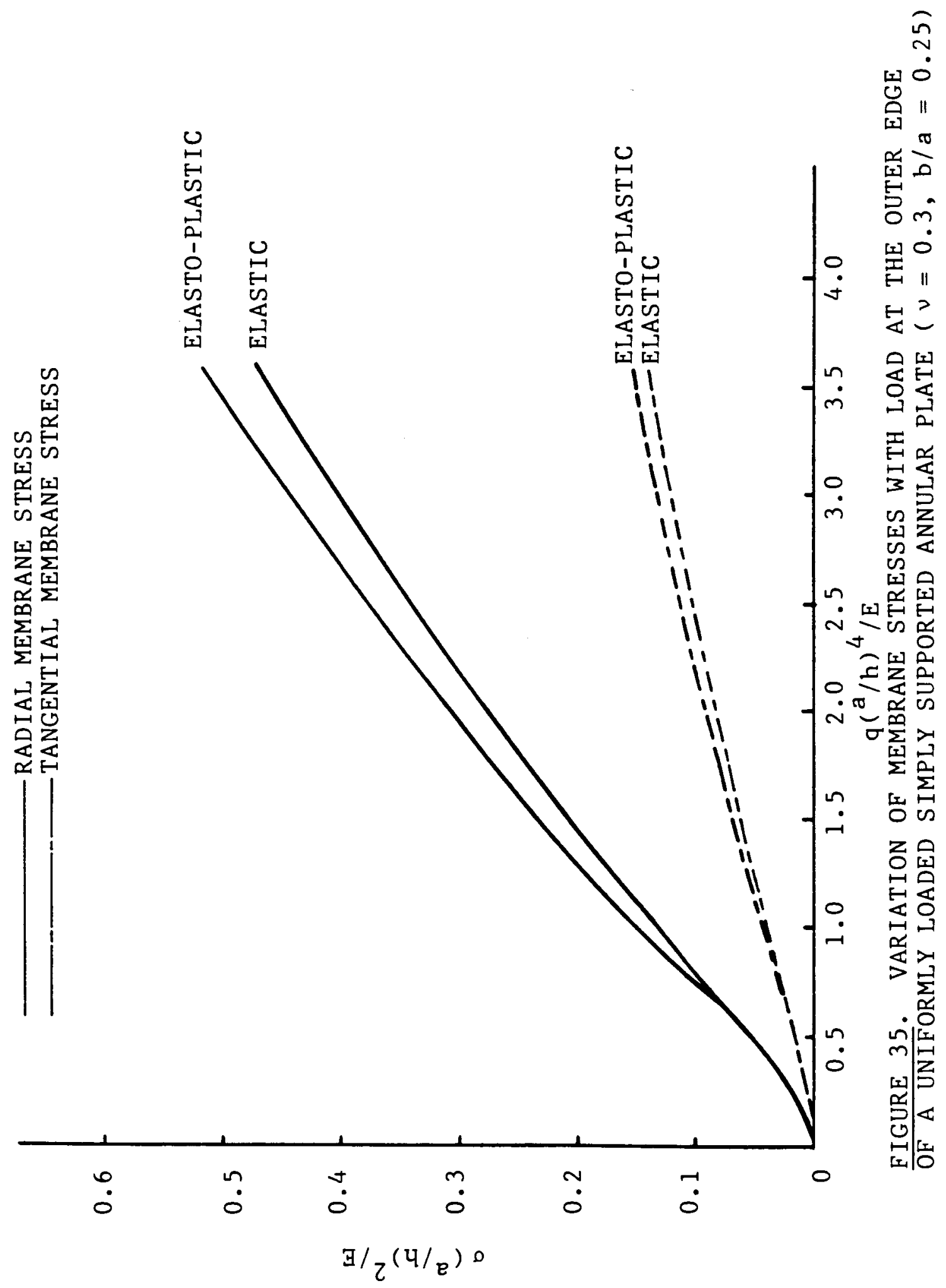


鄂递

중

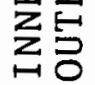

昰㹂

[다 디

$\circlearrowleft 0$

红㕸

ज垸

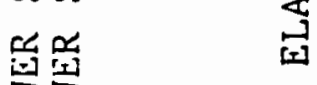

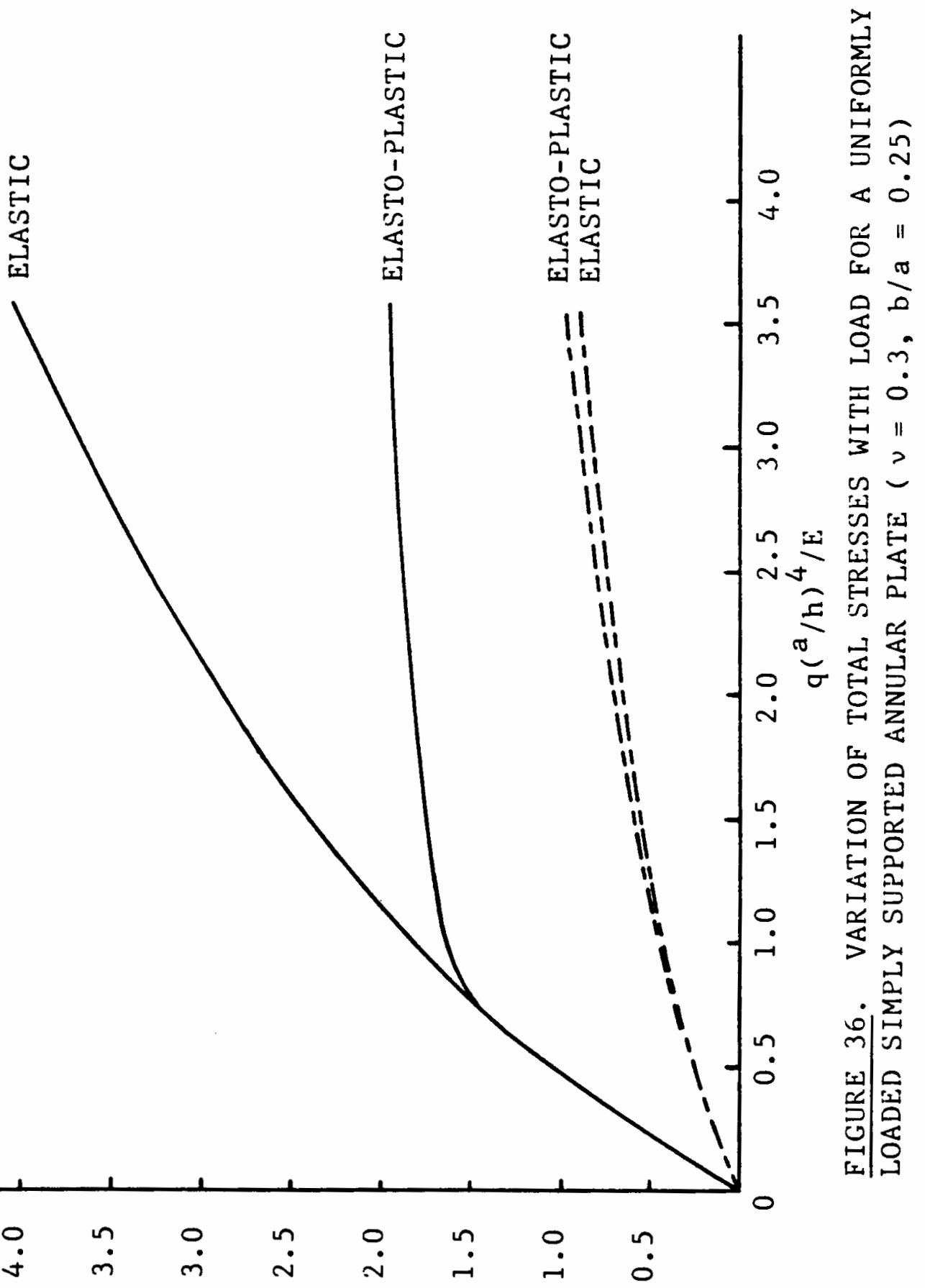

$\exists / \tau(4 / 8) 0$ 


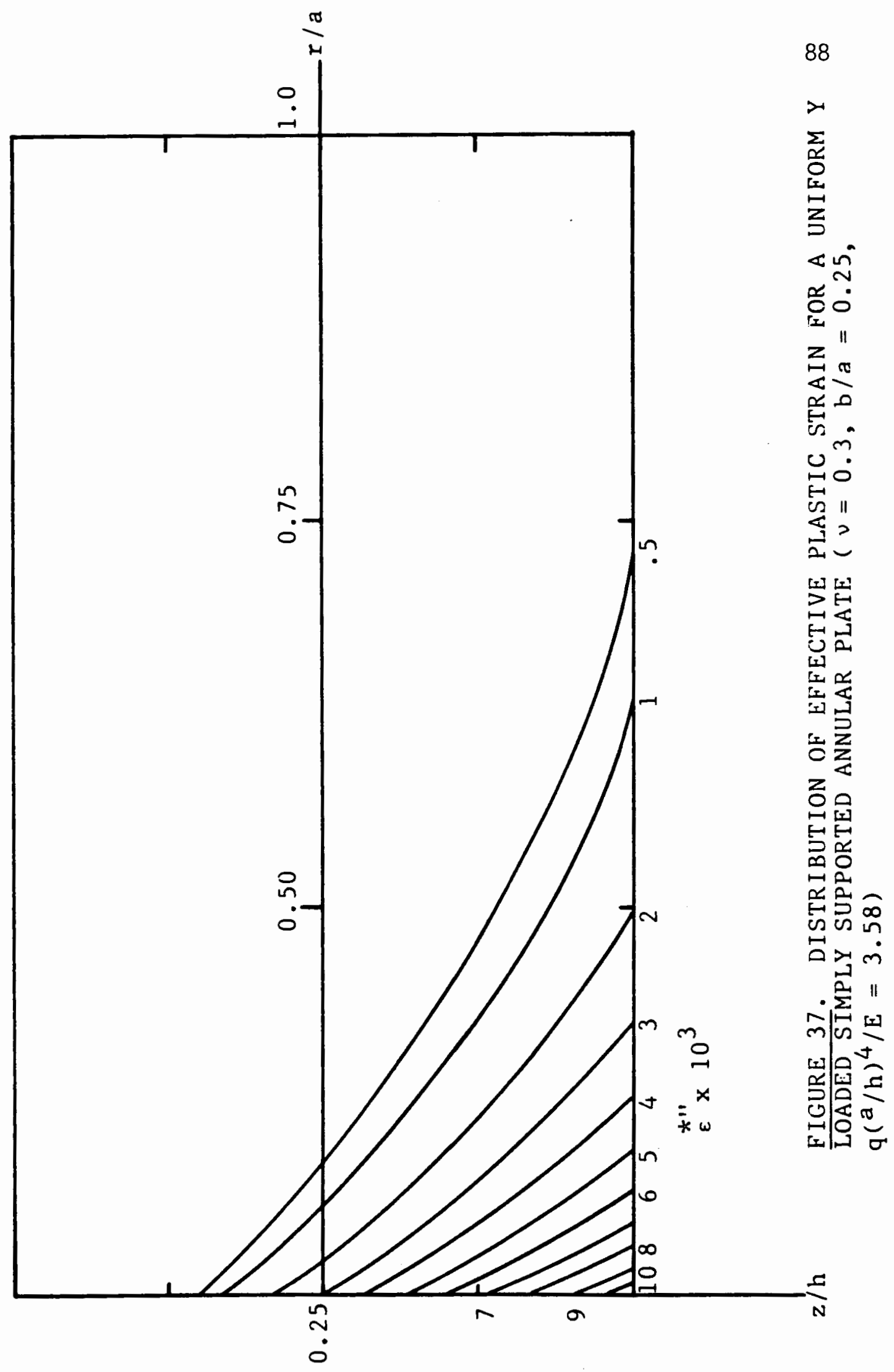

$05^{\circ} 0-$

$\varsigma 2 \cdot 0-$

0

$\varsigma Z^{\circ} 0$

$0 S^{\circ} 0$ 


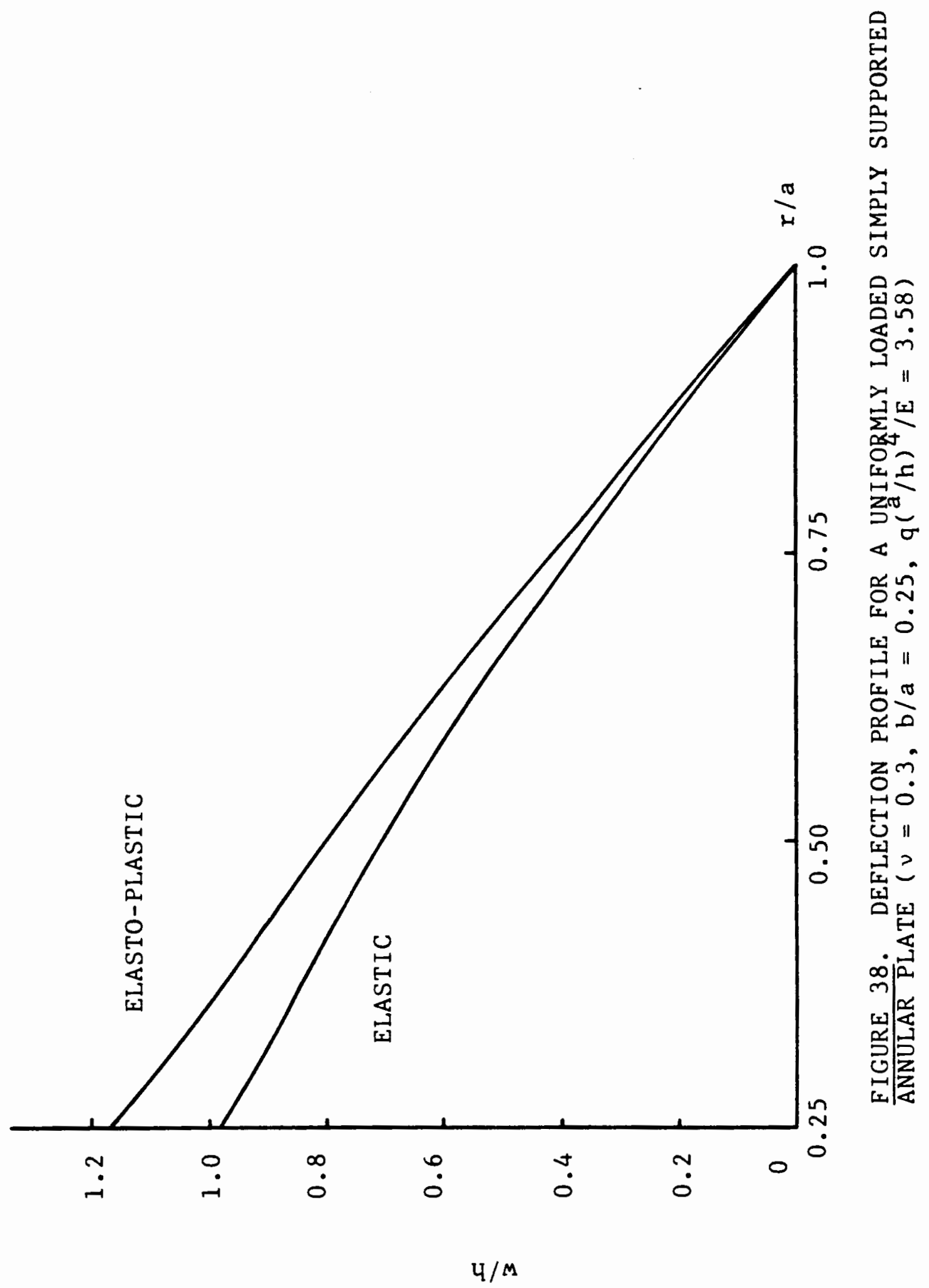


呚

世约

똬

部

啗

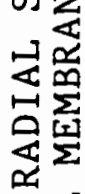

正安

엉 运

11

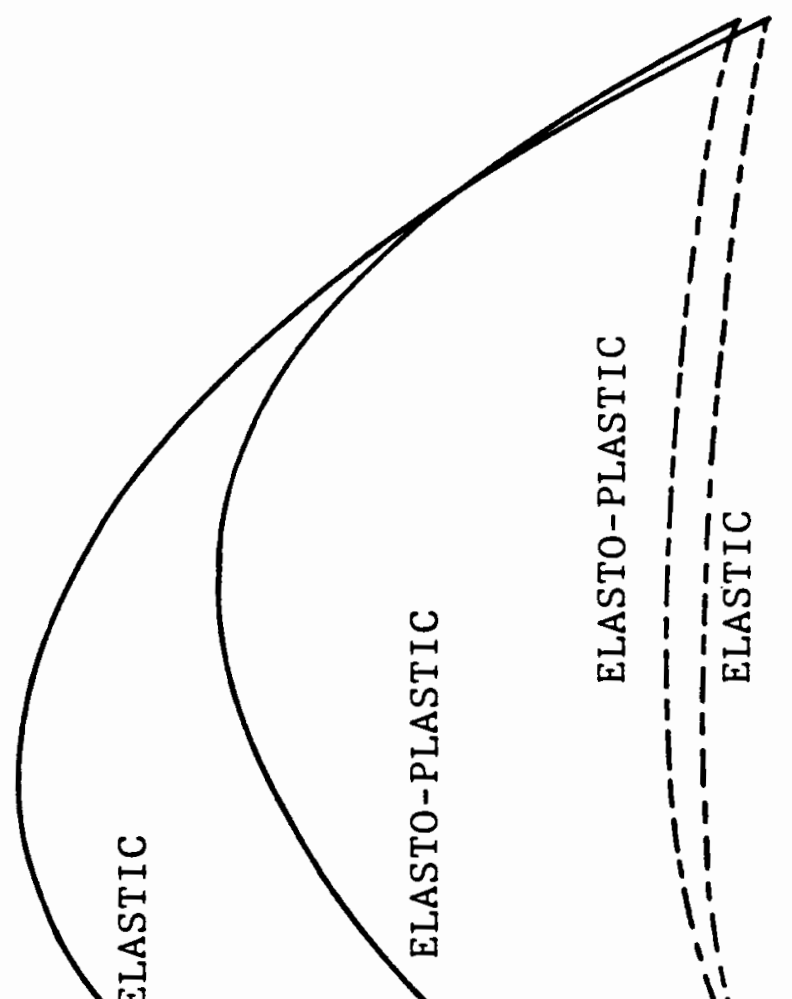

30

韪?

i $\quad \sum_{i=1}$

외

\&र

耐

-

20

完

ํㅗㅇ in

$\because v$

号

$<11$

옹 뭉

ก.

$\circ$ 떱m

曰.

s 0

采 I

c

되 띠

足

봃
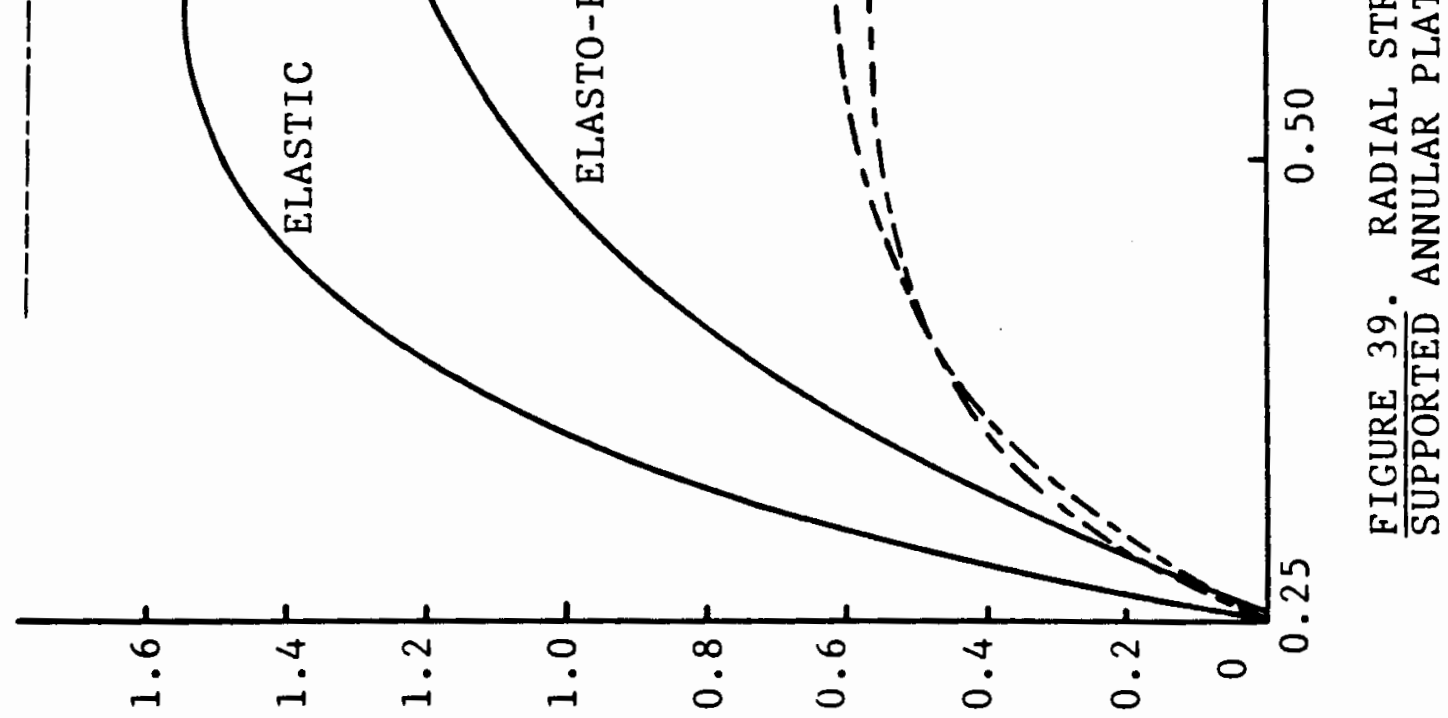

$\exists / 2\left(4 /{ }^{2}\right) 0$ 


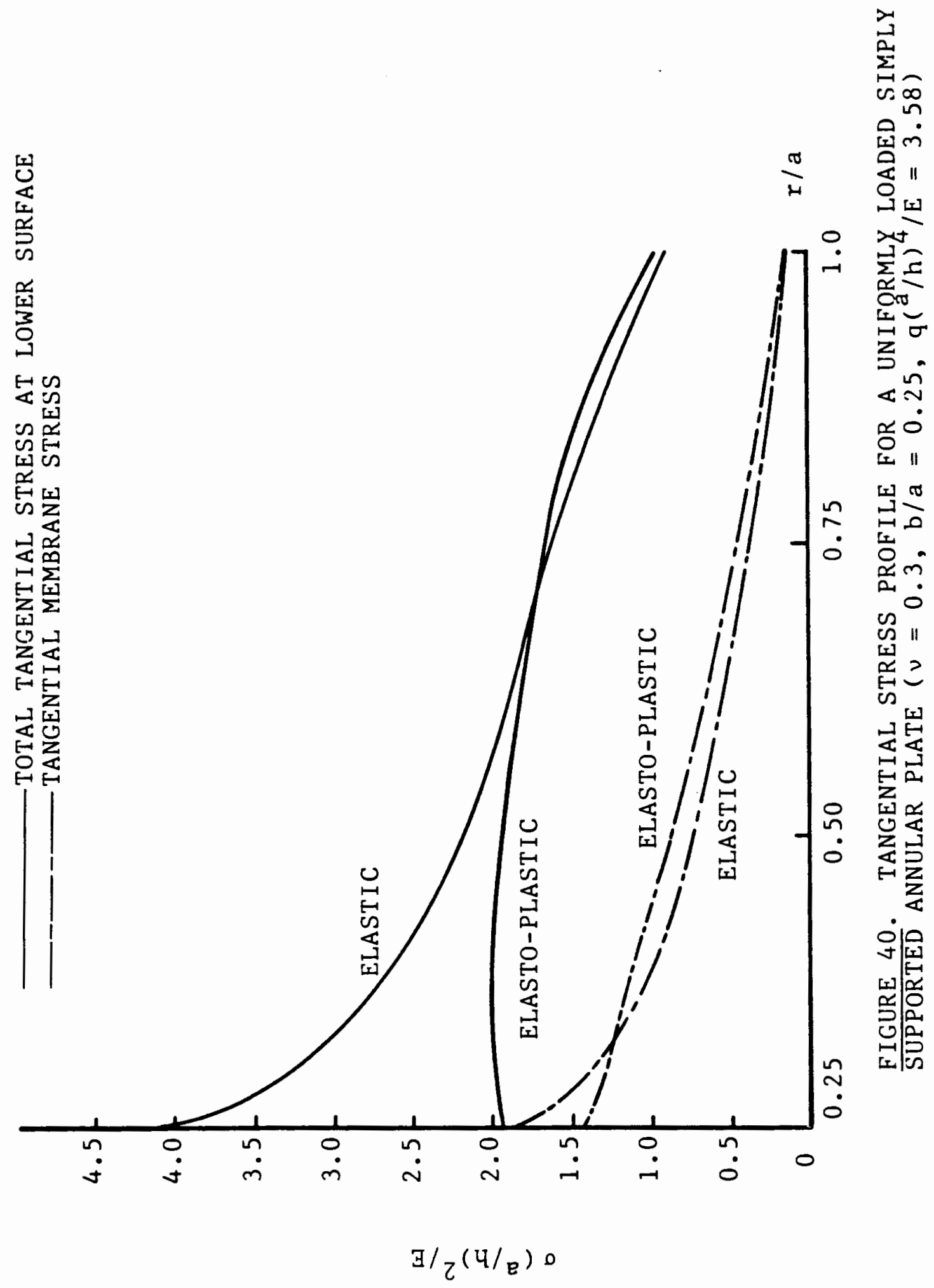




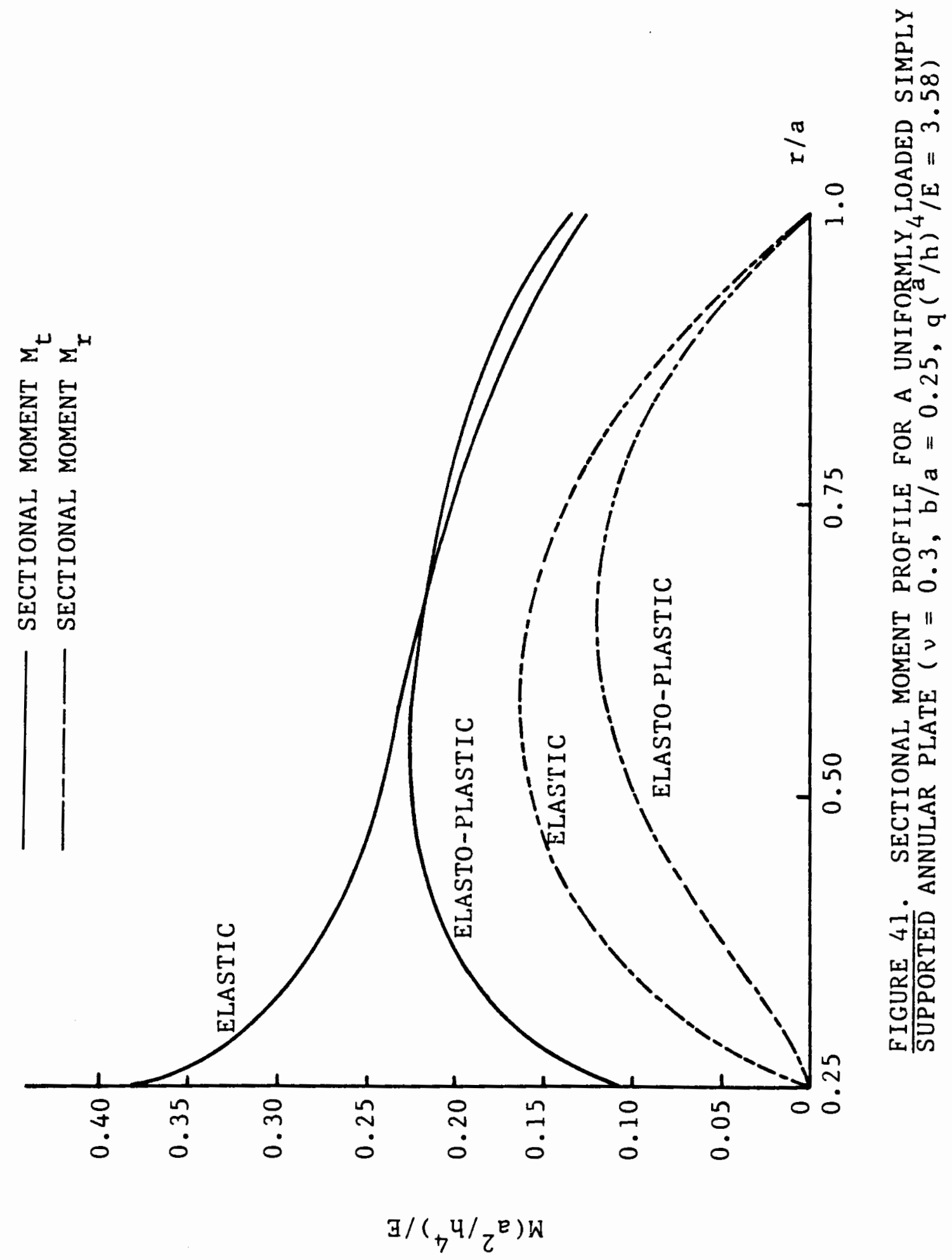




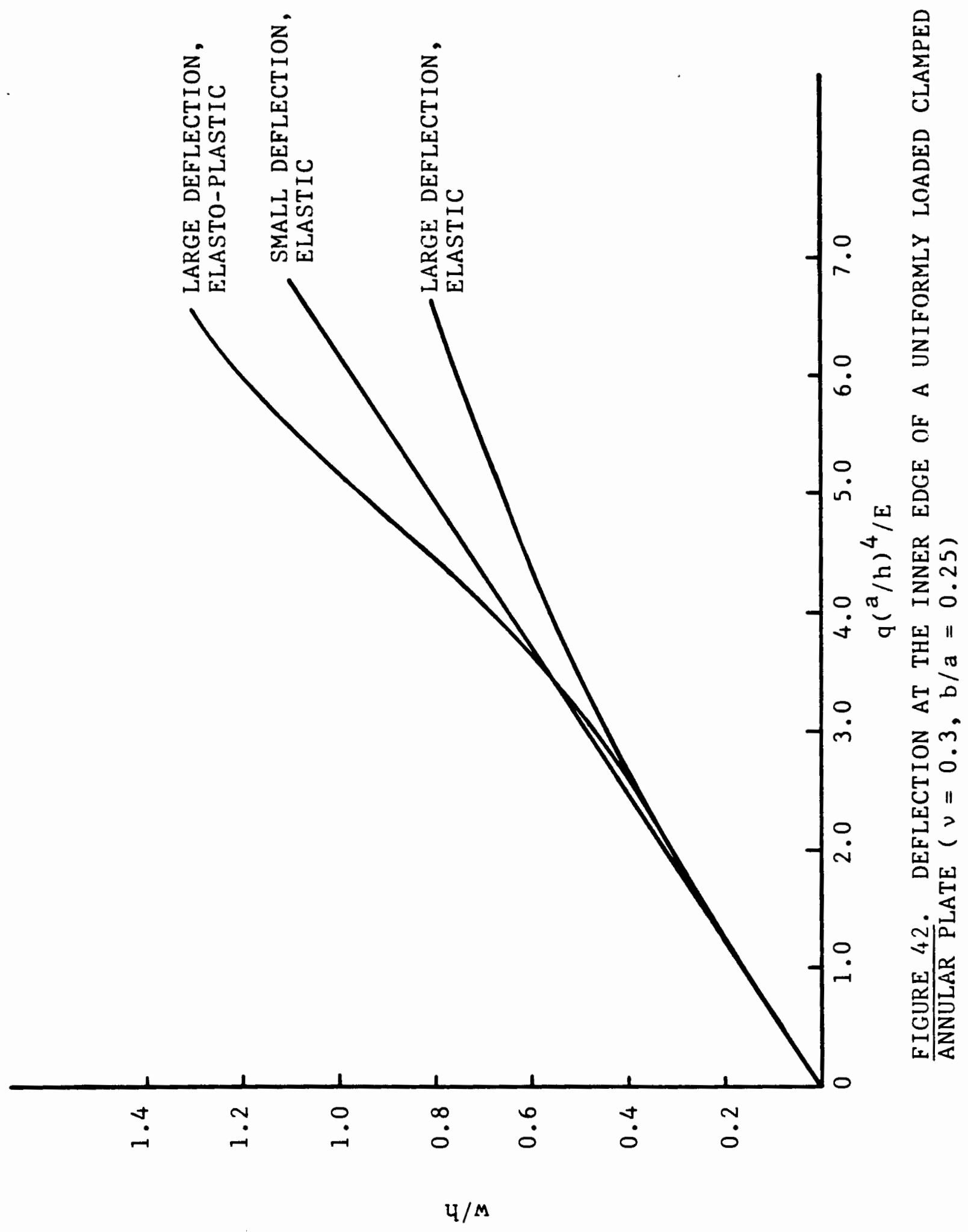




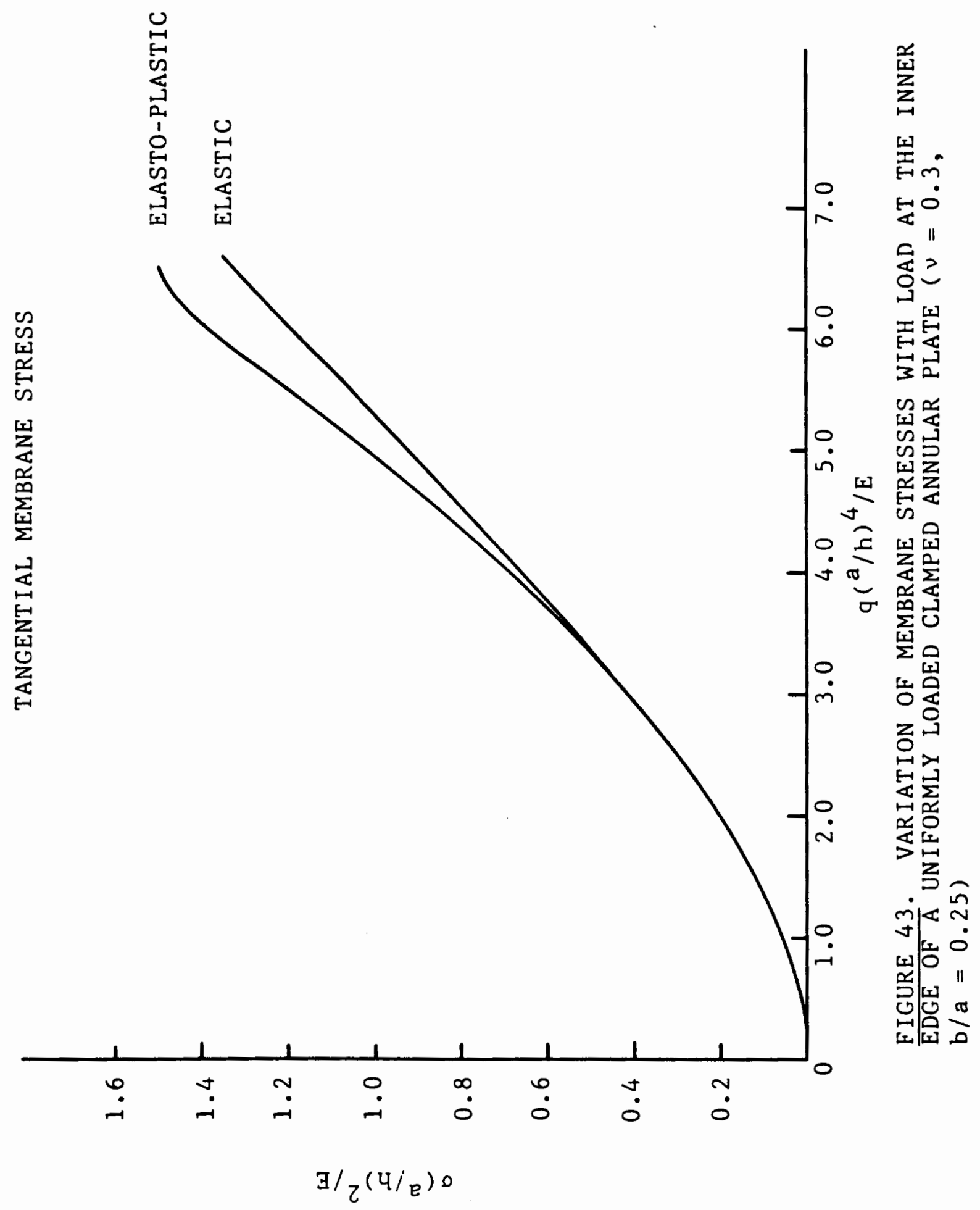




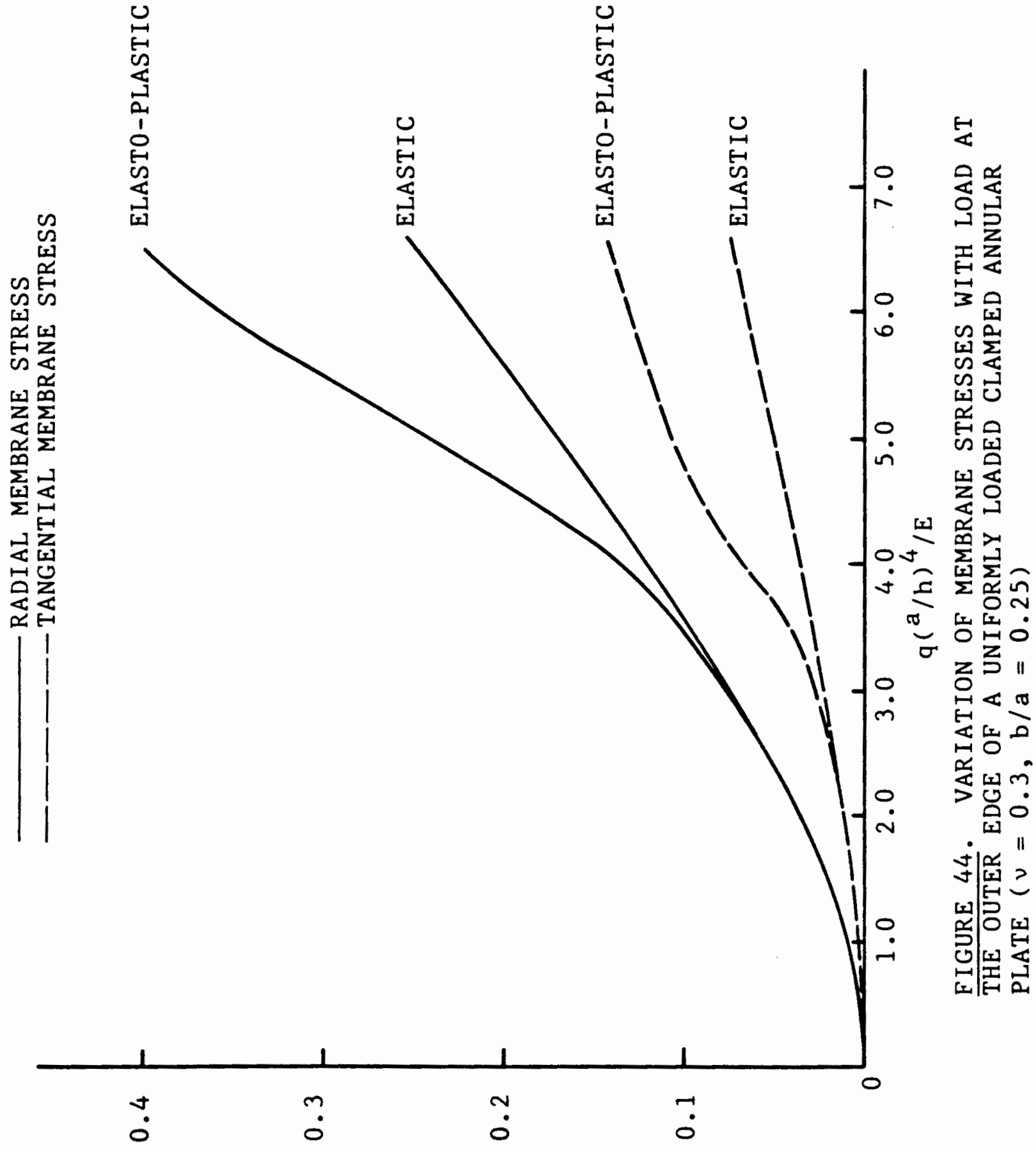

$\exists / Z(4 / 8) 0$ 


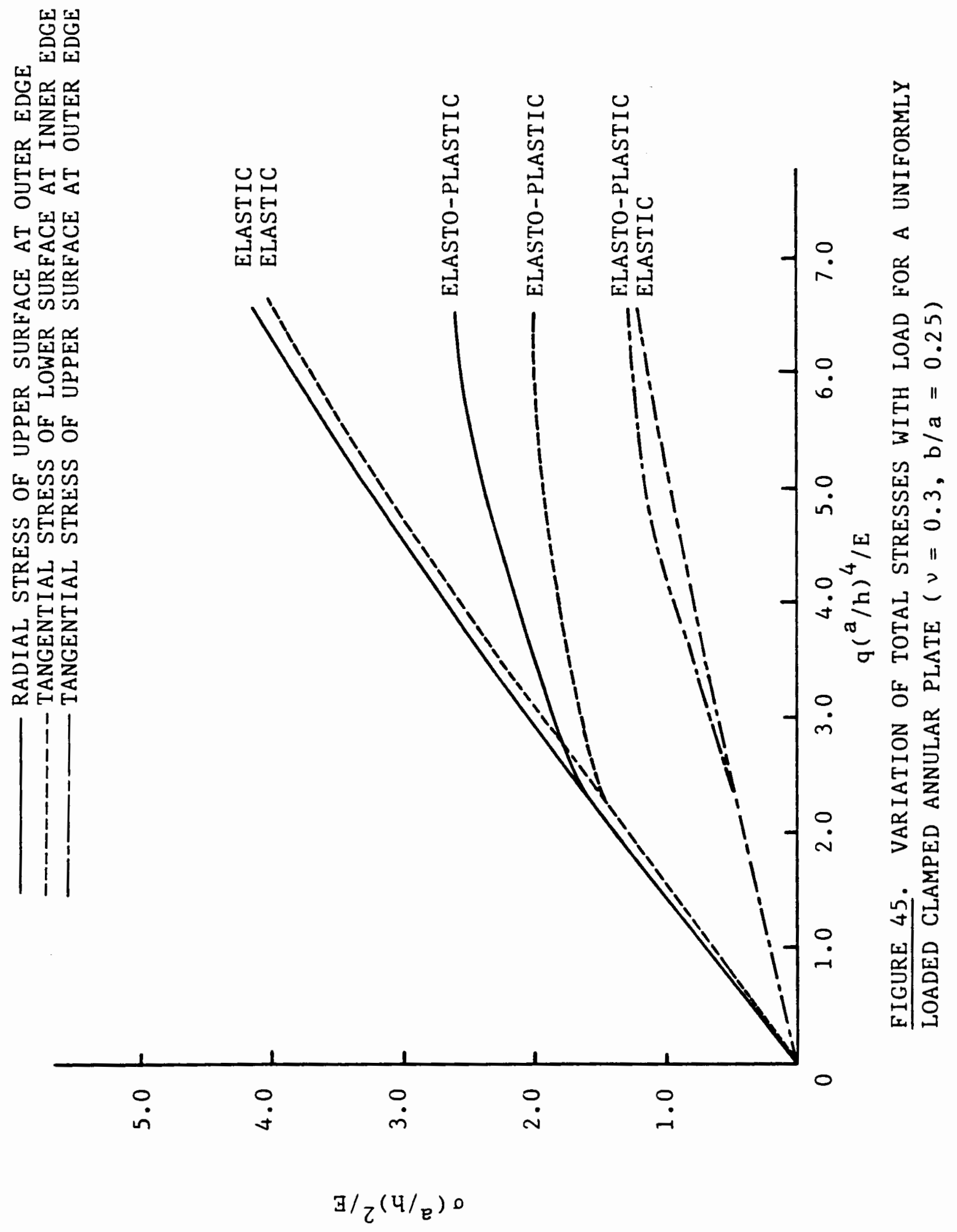




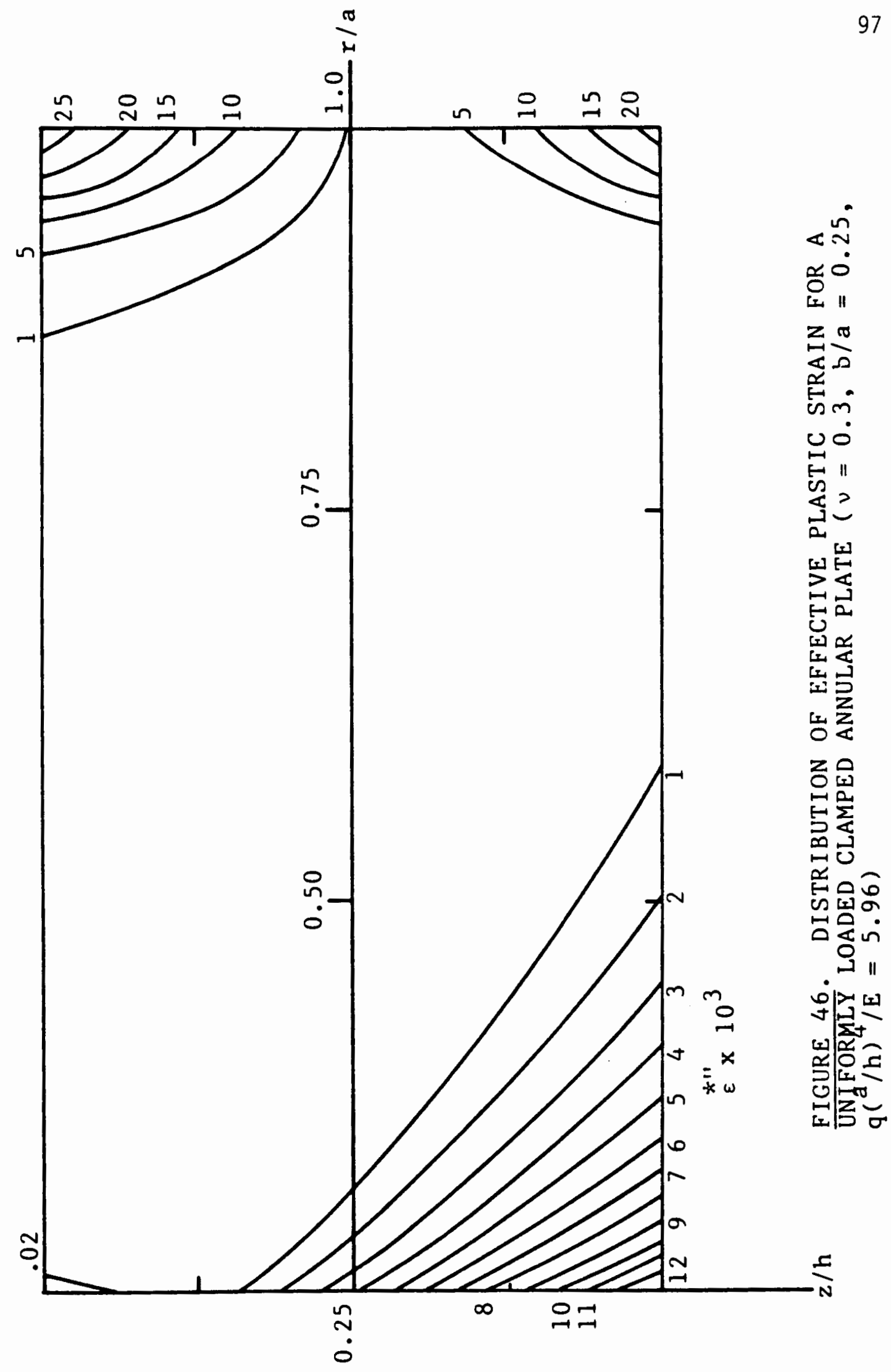

$O S^{\circ} 0-$

$\varsigma 2^{\circ} 0^{-}$

0

$\varsigma \tau^{\circ} 0$

$05^{\circ} 0$ 


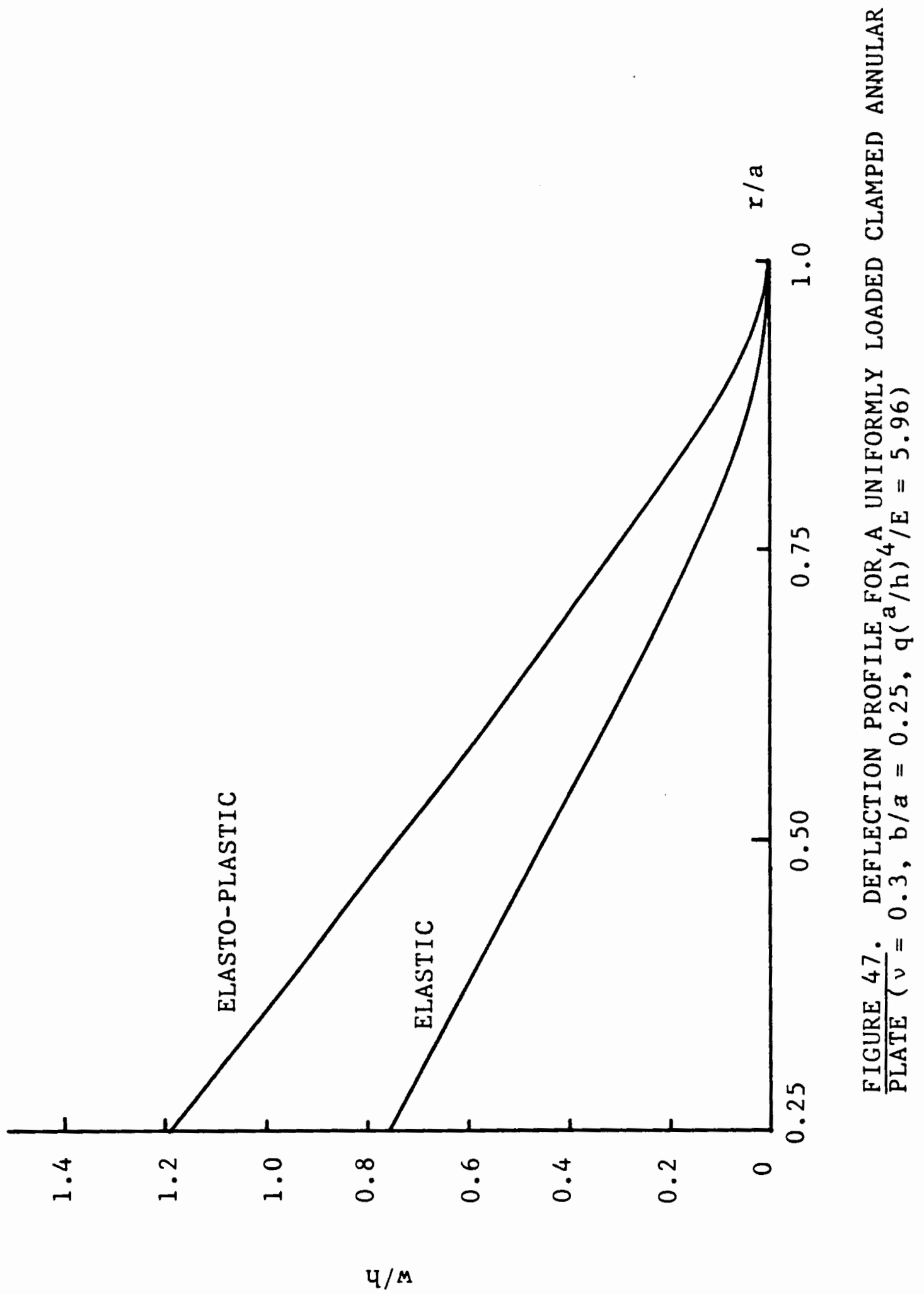




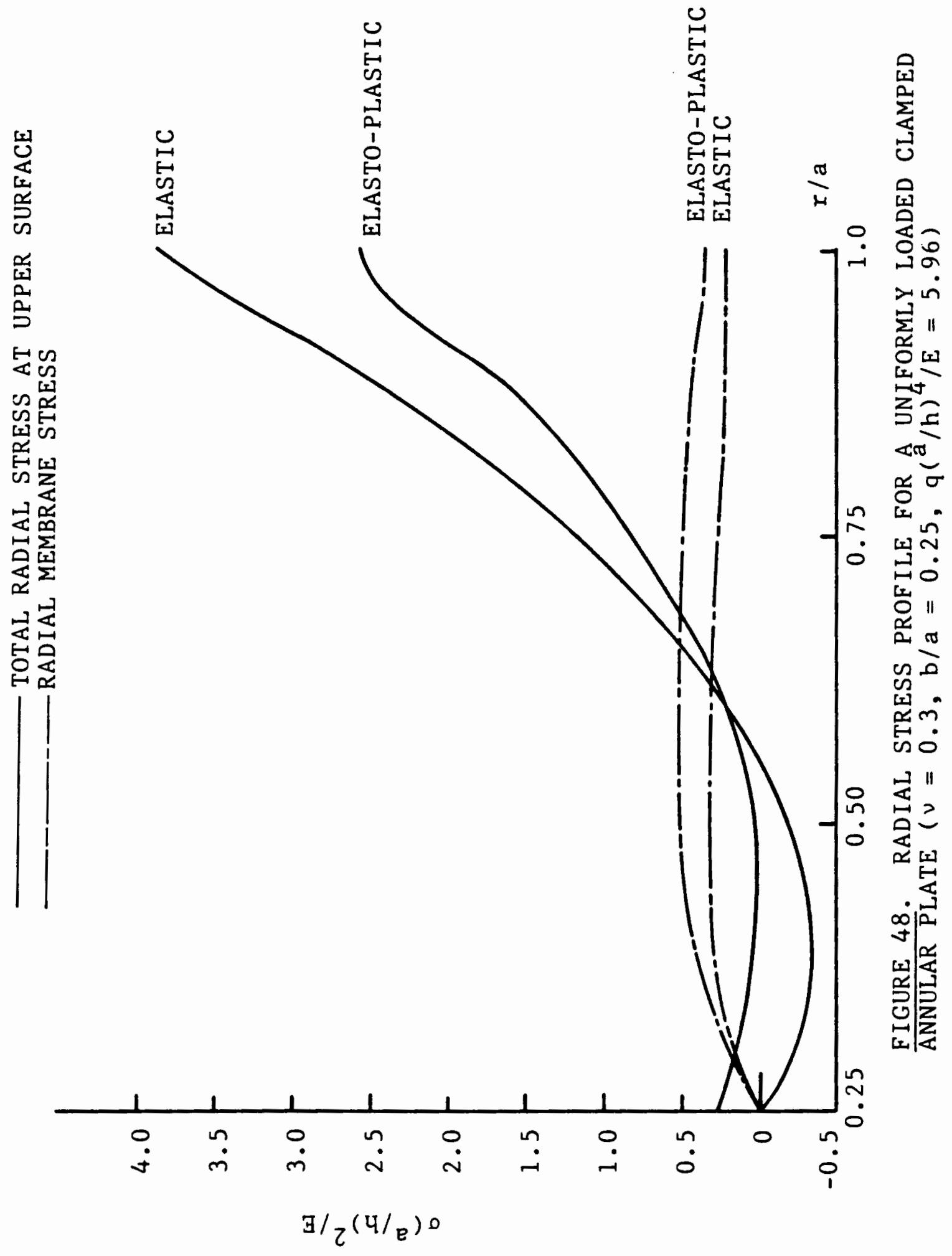




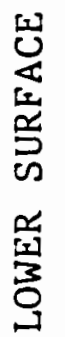

Ex

⿻ 된

N

哟

본

$\rightarrow \frac{1}{\alpha}$

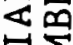

닌

(1)

足是

EE

$\checkmark$ 되

世

$0<$

1

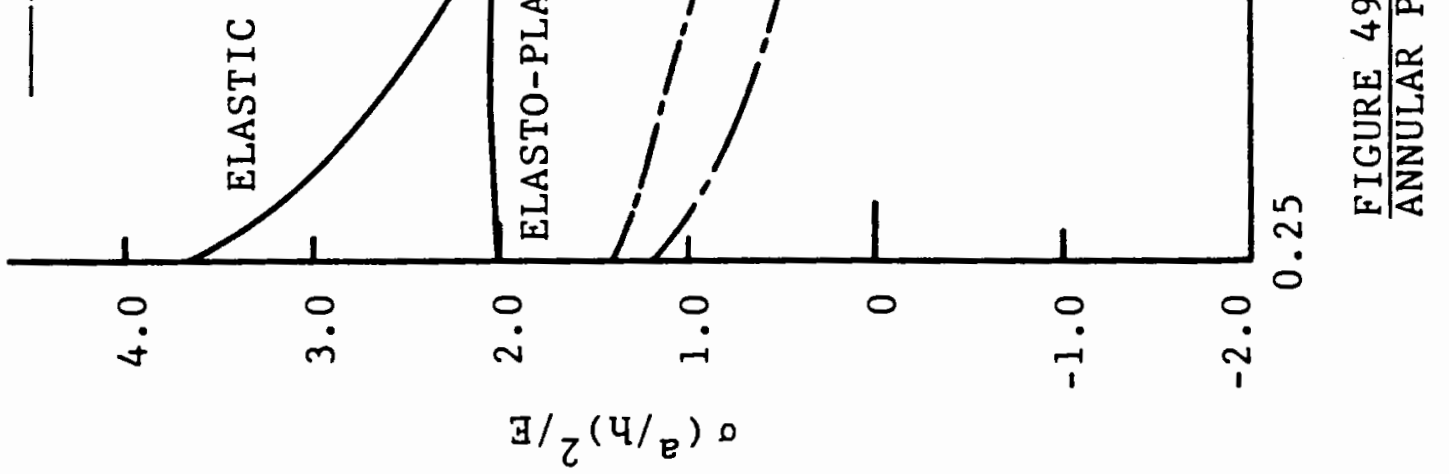




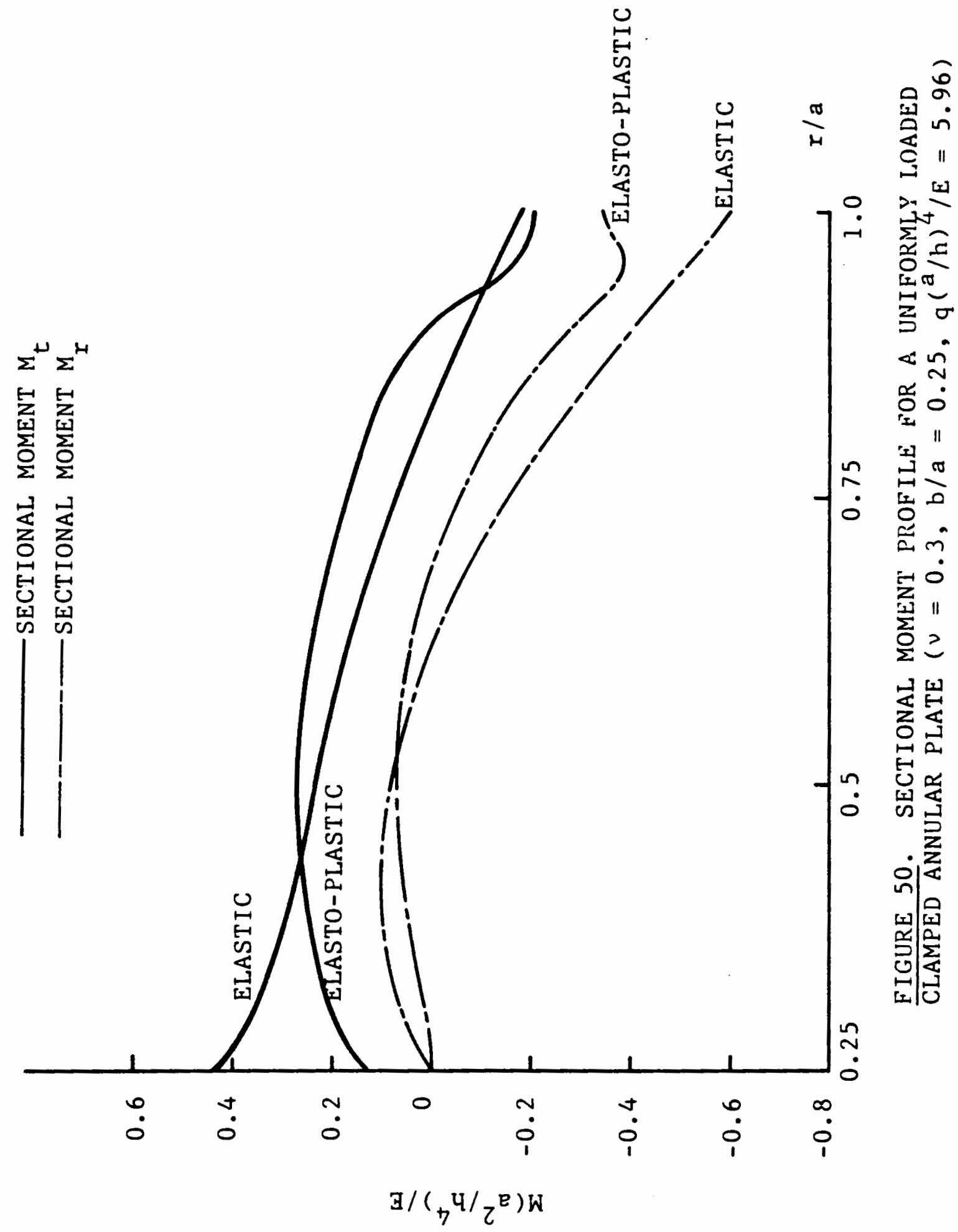


CHAPTER VI

CONCLUSIONS

Solutions of Von Karman type circular and annular plates were obtained by considering equivalent plates with small displacements, where the nonlinear terms of lateral displacement and the plastic strain were treated as fictitious body and surface loads acting on the plate. In this way, solutions for many difficult nonlinear problems may be obtained by using the known solutions of the linear problems through an iteration procedure. The numerical iteration scheme used in this investigation resulted in rapid convergence to the final solutions. The method of solution presented in this study is found to be computationally efficient and offers an alternative method for obtaining the nonlinear solutions to plate bending problems.

Nonlinear elastic results for simply supported and clamped circular and annular plates have been shown to be in excellennt agreement with the available solutions. The results of the nonlinear elastic-plastic solutions show that the lateral displacement is increased by the plastic strain while the maximum fiber stresses are considerably relieved. The consideration of the behavior beyond the elastic limit is important since a high premium is currently being placed on the saving of weight in aircraft, missile, and space applications. 
The procedure used in this study can be employed to solve a variety of nonlinear plate bending problems under different loading and boundary conditions. Furthermore, the present formulation can easily be extended to include other sources of nonlinearity such as creep and relaxation and to obtain solutions for orthotropic plates undergoing large deflections. In concluding this chapter, it is perhaps worth pointing out that the present approach does not require the large computational storage and high cost normally involved in the nonlinear analys is of composite plates by the finite element method (1). The procedure is thus well suited for the nonlinear analysis of this class of annular plates. 


\section{BIBL IOGRAPHY}

1. Gorji, M., "On Large Deflection of Symmetric Composite Plates Under Static Loading," Journal of Mechanical Engineering Science, Proceedings of the Institution of Mechanical Engineers, Part C, Vol. 200, No. C1, 1986, pp. 13-19.

2. Lin, T.H., Theory of Inelastic Structures, John Wiley and Sons, Inc., New York, N.Y., 1968.

3. Timoshenko, S., and Woinowsky-Krieger, S., Theory of Plates and Shells, 2nd ed., McGraw-Hill Book Co., Inc., New York, N.Y., 1959.

4. Von Karman, T., "Festigkeits Probleme Im Maschinenbau," Encyklopaedie Der Mathematischen Wissenschaften, Germany, Vol. 4, 1910, pp. 348-352.

5. Way, S., "Bending of Circular Plates with Large Deflection," Journal of Applied Mechanics, Vol. 56, 1934, pp. 627-636.

6. Alwar, R.S., and Nath, Y., "Applications of Chebyshev Polynomials to the Nonlinear Analys is of Circular P lates, "International Journal of Mechanical science, Vol. 18, 1976, pp. 589-595.

7. Alwar, R.S., and Reddy, B.S., "Large Deflection Static and Dynamic Analys is of Isotropic and Orthotropic Annular Plates," International Journal of Non-Linear Mechanics, Vol. 14, 1979, pp. 347-359.

8. Berger, H.M., "A New Approach to the Analys is of Large Deflections of Plates," Journal of Applied Mechanics, Vol. 22, 1955, pp. $465-472$.

9. Federhofer, K., and Egger, H., "Berechnung der dunnen Kreisplatte mit grosser Ausbiegung," Sitzungsberichte der Akademischen Wissenschaften, Vienna, Austria, Vol. 155, 1946, pp. 15-43.

10. Mansfield, E.H., The Bending and Stretching of Plates, Pergamon Press, 1964.

11. Stoker, J.J., Nonlinear Elasticity, Gordon and Breach Science Publishers, 1968. 
12. Keller, H.B., and Reiss, E.L., "Nonlinear Bending of Circular Plates," Proceedings of the Third National Congress of Applied Mechanics, Providence, RI, 1958, pp. 375-385.

13. Mah, G.B.J., "Axisymmetric Finite Deflection of Circular Plates," Journal of the Engineering Mechanics Divison, ASCE, Vol. 95, 1969, pp. 1125-1143.

14. Furlong, R.W., Becker, E.B., and Colville, J., "Large Displacement Analys is of Thin Plates," Journal of Structural Engineering, ASCE, Vol. 99, No. ST3, 1973, pp. 349-364.

15. Murthy, S.D.N., and Sherbourne, A.N., "Nonlinear Bending of Elastic Plates of Variable Profile," Journal of Engineering Mechanics Division, ASCE, Vol. 100, 1974, pp. 251-265.

16. Turvey, G.J., "Large Deflection of Tapered Annular Plates by Dynamic Relaxation," Journal of the Engineering Mechanics Division, ASCE, Vol. 104, 1978, pp. 351-366.

17. Chien, W.Z., "Large Deflections of a Circular Clamped Plate Under Uniform Pressure," Chinese Journal of Physics, Vol. 7, 1947, pp. 102-114.

18. Chien, W.Z., and Yeh, K.Y., "On the Large Deflection of Circular Plates," Acta scient. Sin., Vol. 3, 1954, pp. 405-436.

19. Schmidt, R., "Large Deflections of a Clamped Circular Plate," Journal of the Engineering Mechanics Division, ASCE, Vol. 94, 1968, pp. $1603-1606$.

20. Schmidt, R., "Finite Deflections of a Loosely Clamped Circular Plate Loaded at its Center," Journal of Indust. Math. Society, Vol. 23, Part 1, 1973, pp. 45-51.

21. Schmidt, R., and Da Deppo, D.A., "Several Perturbation Solutions in the Nonlinear Theory of Circular Plates and Membranes," Journal of Indust. Math. Society, Vol. 25, Part 2, 1975, pp. 83-96.

22. Stippes, M., and Hausrath, A.H., "Large Deflection of Circular Plates," Journal of Applied Mechanics, Vol. 19, 1952, pp. 287-292.

23. Krayterman, B.L., and Fu, C.C., "Nonl inear Analys is of $\mathrm{Cl}$ amped Circular Plates," Journal of Structural Engineering, ASCE, Vol. 111, 1985, pp. 2402-2415.

24. Batternam, S.C., and Lehner, J.R., "Nonlinear Static and Dynamic Deformations of Shells of Revolution," International Journal of Nonlinear Mechanics, Vol. 9, 1974, pp. 501-519. 
25. Dumir, P.C., Gandhi, M.L., and Nath, Y., "Nonlinear Static and Transient Analys is of Orthotropic Thin Circular Plates with Elastically Restrained Edge under Central Load," Journal of Composite Materials, Vol. 17, 1983, pp. 478-491.

26. Dumir, P.C., and Shingal, L., "Nonl inear Analys is of Thick Circular Plates," Journal of Engineering Mechanics Division, ASCE, Vol. $112,1986, \mathrm{pp} .260-272$.

27. Nath, Y., Dumir, P.C., and Bhatia, R.S., "Nonlinear Static and Dynamic Analysis of Circular Plates and Shallow Sperical Shells Using the Collocation Method," International Journal for Numerical Methods in Engineering, Vol. 21, 1985, pp. 565-578.

28. Wempner, G.A., and Schmidt, R., "Large Symmetric Deflections of Annular Plates," Journal of Applied Mechanics, Vol. 25, 1958, pp. 449-452.

29. Yeh, K.Y., "Large Deflection of a Circular Plate with a Circular Hole at the Center," Acta Scient. Sin., Vol. 2, 1953, pp. 127-144.

30. Dumir, P.C., Nath, Y., and Gandhi, M.L., "Non-Linear Axisymmetric Stat ic Analysis of Orthotropic Thin Annular Plates," International Journal of Non-Linear Mechanics, Vol. 19, 1984, pp. 255-272.

31. Sokolovsky, V.V., "Elastic-Plastic Bending of Circular and Annular Plates," Prikladnaya Mate matika i Mekhanika, Moscow, U.S.S.R., Vol. 8, No. 2, 1944, pp. 141-166.

32. Sokolovsky, V.V., "Elasto-PIastic Bending of Circular and Annular Plates," Brown University, Division of Applied Mathematics, Technical Report No. 3, 1955.

33. Grigoriev, A.S., "Bending of Circular and Annular Plates with Variable Thickness Beyond the Elastic Limit," Inzherneryi Shornik, Moscow, U.S.S.R., Vol. 20, 1954, pp. 52-92.

34. Dvorak, J., "Circular Ring Plate in Elastic-Plastic State," Proceedings, Non-Homogeneity in Elasticity and Plasticity, Warsaw, 1959, pp. 519-521.

35. Ohashi, Y., and Murakami, S., "On the Elastic-Plastic Bending of a Clamped Circular Plate Under a Partial Circular Uniform Load," Bulletin, Japan Society of Mechanical Engineers, Vol. 7, No. 27, 1964, pp. 491-498. 
36. Drucker, D.C., and Hopkins, H.G., "Combined Concentrated and Distributed Load on Ideally-Plastic Circular Plates,"

Proceedings, 2nd U.S. National Congress of Applied Mechanics, Ann Arbor, MI, 1954, pp. 517-520.

37. Hopkins, H.G., and Prager, W., "The Load-Carrying Capacities of Circular Plates," Journal of the Mechanics and Physics of Solids, London, Vol. 2, 1953, pp. 1-13.

38. Pell, W.H., and Prager, W., "Limit Design of Plates," Proceedings, 1st U.S. National Congress of Applied Mechanics, Chicago, IL, 1951, pp. 547-550.

39. Haythornthwaite, R.M., "Deflection of Plates in Elastic-Plastic Range," Proceedings, 2nd U.S. National Congress of Applied Mechanics, Ann Arbor, MI, 1954, pp 521-526.

40. Lackman, L.M., "Circular Plates Loaded into the Plastic Region," Journal of the Engineering Mechanics Division, ASCE, Vol. 90, №. EM6, 1964, pp. 21-30.

41. Lin, T.H., "Bending of a Plate with Nonlinear Strain Hardening Creep," Proceedings of the Colloquium on Creep Structures, International Union of Applied Mechanics, Julius Springer, Berlin, 1962, pp. 215-228.

42. Popov, E.P., Khojesteh-Bakht, M., and Yaghmai, S., "Analysis of Elastic Plastic Circular Plates," Journal of the Engineering Mechanics Division, ASCE, Vol. 93, 1967, pp. 49-65.

43. Turvey, G.J., and Lim, G.T., "Axisymmetric Full-Range Analys is of Transverse Pressure-Loaded Circular Plates," International Journal of Mechanical Science, Vol. 26, No. 9110, 1984, pp. 489-502.

44. Naghdi, P.M., "Bending of Elastoplastic Circular Plates with Large Deflection," Journal of Applied Mechanics, ASME, Vol. 19, 1952, pp. 293-300.

45. Ohashi, Y., and Murakami, S., "Large Deflection in Elastoplastic Bending of a Simply Supported Circular Plate Under a Uniform Load," Journal of Applied Mechanics, ASME, Vol. 33, pp. 866-870.

46. Ohashi, Y., and Murakami, S., "The Elasto-Plastic Bending of a Clamped Thin Circular Plate," Proceedings of the 11th Interational Congress of Applied Mechanics, Munich, 1964, pp. 212-223.

47. Ohashi, Y., Murakami, S., and Endo, A., "El asto-Plastic Bending of an Annular Plate at Large Deflection," Ingenieur-Archiv, Vol. 35, 1967, pp. 340-350. 
48. Ohashi, Y., and Murakami, S., "Axisymmetric Elasto-Plastic Deformation of Circular Plates Under Combined Action of Lateral Load and Membrane Force," Memories of the Faculty of Engineering, Nagoya University, Vol. 21, 1969, pp. 79-121.

49. Myszkowski, J., "Endliche Durchbiegungen Beliebig Eingspannter Dunner Kreis-und Kreisring Platten Im Plastischen Materialbereich," Ingenieur-Archiv, Vol. 40, 1971, pp. 1-13.

50. Turvey, G.J., "Axisymetric Elasto-Plastic Flexure of Circular Plates in the Large Deflection Regime," Proceedings of the Institution of Civil Engineers, Part 2, Vol. 67, 1979, pp. 81-92.

51. Turvey, G.J., "Thickness-Tapered Circular Plates, An Elasto-Plastic Large Deflection Analysis," Journal of Structural Mechanics, Vol. 7, 1979, pp. 247-271.

52. Sherbourne, A.N., and Srivastava, N.K., "Elastic-Plastic Bending of Restrained Pin-Ended Circular Plates," International Journal of Mechanical Science, Pergamon Press, Vol. 13, 1971, pp. 231-241.

53. Hill, R., Mathematical Theory of Plasticity, Oxford University Press, London, 1950.

54. Kachanov, L.M., Foundation of the Theory of Plasticity, North-Holland Publishing Co., Amsterdam, 1971.

55. Mendelson, A., Plasticity: Theory and Application, The Macmillan Company, New York, NY, 1968.

56. Crose, J.G., and Ang, A.H.S., "Nonlinear Analysis Method for Circular Plates," Journal of the Engineering Mechanics Division, ASCE, Vol. 95, 1969, pp. 979-999.

57. Onat, E.T., and Haythornthwaite, R.M., "The Load Carrying Capacity of Circular Plates at Large Deflection," Journal of Applied Mechanics, Vol. 23, 1956, pp. 49-55.

58. Tanaka, M., "Large Deflection Analys is of El astic-Plastic Circular Plates with Combined Isotropic and Kinematic Hardening," Ingenieur-Archiv, Vol. 41, 1972, pp. 342-356.

59. Hamada, M. and Tanaka, M., "A Numerical Method Considering the Bauschinger Effect for Large Deflection Analys is of Elastic-Plastic Circular Plates," Bulletin, Japanese Society of Mechanical Engineers, Vol. 15, No. 87, 1972, pp. 1029-1040.

60. Gorji, M., "Nonlinear Analys is of Plates with Plastic Orthotropy," Journal of Structural Engineering, ASCE, Vol. 3, No. 10, 1985, pp. 2214-2226. 
61. Lin, T.H., Lin, S.R., and Mazelsky, B., "Elastic-Plastic Bending of Rectangular Plates with Large Deflection," Journal of Applied Mechanics, ASME, Vol. 39, 1972, pp. 978-982.

62. Lin, T.H., "On the Associated Flow Rules of Plasticity Based on Crystal Slips," Journal of the Franklin Institute, Vol 270, No. $4,1960$.

63. Lin, T.H., and Ho, E., "Elasto-Plastic Bending of a Rectangular Plate," Journal of Engineering Mechanics Division, ASCE, Vol. 94, EM1, 1968, pp. 199-210.

64. Zienkiewicz, O.C., The Finite Element Method, 3rd ed., McGraw-Hill Book Co., Inc., London, 1977.

65. Ugural, A.C., Stresses in Plates and Shells, McGraw-Hill Book Co., Inc., New York, NY, 1981.

66. Stockton, F.D., and Drucker, D.C., "Fitting Mathematical Theories of Plasticity to Experimental Results, Journal of Colloid Sciences (Rheology issue), Vol. 5, 1950, pp. 239-250.

67. Ramberg, W., and Osgood, W.R., "Description of Stress-Strain Curves by Three Parameters," NACA TN 902, 1943. 
APPENDICES 
APPENDIX A

NOTATION

The following symbols are used in this study:

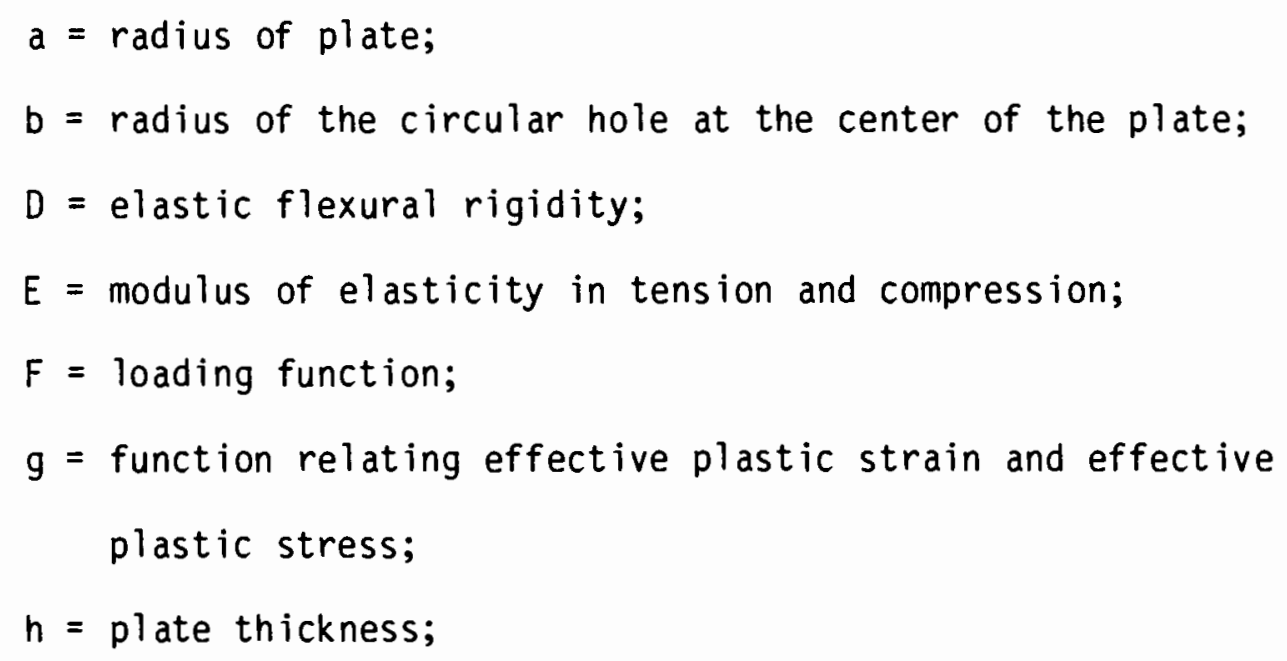


$\bar{N}_{r}, \bar{N}_{t}=$ sectional forces per unit length due to geometric nonlinearity;

$N_{r}^{\prime \prime}, N_{t}^{\prime \prime}=$ inelastic sectional forces per unit length;

$N_{r}^{E}, N_{t}^{E}=$ equivalent sectional forces per unit length;

$n=$ constant;

$Q_{r}=$ shearing force per unit length of the cylindrical section of radius $r$;

$Q_{r}^{\prime \prime}=$ inelastic shearing force per unit length of the cylindrical section of radius $r$;

$q=$ intensity of uniformly distributed lateral load;

$\bar{q}=$ intensity of fictitious lateral load due to nonlinear terms of lateral displacement;

$q "=$ intensity of fictitious lateral load due to the plastic strains;

$\bar{R}=$ radial component of intensity of fictitious body force due to nonlinear terms of lateral displacement;

$R^{\prime \prime}=$ radial component of intensity of fictitious body force due to the plastic strains;

$r, t=$ radial and tangential cylindrical coordinates;

$u, w=$ displacement components along radial and lateral directions;

$z=$ lateral coordinates;

$\varepsilon_{r}, \varepsilon_{t}=$ radial and tangential components of total strain;

$\varepsilon_{r}^{e}, \varepsilon_{t}^{e}=$ radial and tangential components of elastic strain; 
$\varepsilon_{r}^{\prime \prime}, \varepsilon_{t}^{\prime \prime}=$ radial and tangential components of plastic strain;

$\stackrel{\star}{\varepsilon}=$ effective plastic strain;

$d_{\varepsilon}^{\star \prime \prime}=$ effective plastic strain increment;

$d \varepsilon_{i j}=$ components of incremental plastic strain tensor;

$S_{i j}=$ components of deviatoric stress tensor;

$\mathrm{d} \lambda=$ positive constant of proportionality;

$\nu=$ Poisson's ratio;

$\sigma_{11}, \sigma_{22}, \sigma_{33}=$ normal stress components in general coordinates;

$\sigma_{12}, \sigma_{13}, \sigma_{23}=$ shear stress components in general coordinates;

$\sigma_{r}, \sigma_{t}=$ radial and tangential components of stress;

$\stackrel{\star}{\sigma}=$ effective stress;

$d{ }^{\star}=$ effective stress increment;

$\sigma_{y}=$ yield stress in simple tension;

$\sigma_{0}=$ constant;

$\alpha=$ convergance parameter;

$\phi=$ slope; and

$k=$ curvature. 


\section{APPENDIX B}

\section{ANALYTICAL SOLUTIONS OF LINEAR PLATE}

\section{BENDING PROBLEMS}

The small deflection elastic solutions of isotropic circular and annular bending plate problems with various loading conditions of interest for this study are presented herein.

Simply supported circular plates $(3,65)$

A. Subjected to a uniformly distributed lateral load

$$
w=\frac{q}{64 D}\left(a^{2}-r^{2}\right)\left(\frac{5+v}{1+v} a^{2}-r^{2}\right)
$$

B. Subjected to a concentrated load at the center

$$
w=\frac{P}{16 \pi D}\left[\frac{3+v}{1+v}\left(a^{2}-r^{2}\right)+2 r^{2} \operatorname{Ln} \frac{r}{a}\right]
$$

C. Subjected to a uniformly distributed line load at $r=b$

I. Outer portion solution $r>b$

$$
w=\frac{P}{8 \pi D}\left[\left(a^{2}-r^{2}\right)\left(1+\frac{1}{2} \frac{1-v}{1+v} \frac{a^{2}-b^{2}}{a^{2}}\right)+\left(b^{2}+r^{2}\right) \operatorname{Ln} \frac{r}{a}\right]
$$


II. Inner portion solution $r<b$ and $r=b$

$$
w=\frac{p}{8 \pi D}\left[\left(b^{2}+r^{2}\right) \ln \frac{b}{a}+\left(a^{2}-b^{2}\right) \frac{(3+v) a^{2}-(1-v) r^{2}}{2(1+v) a^{2}}\right]
$$

D. Subjected to a uniformly distributed edge moment

$$
w=\frac{M}{2 D(1+v)}\left(a^{2}-r^{2}\right)
$$

$$
\text { Clamped circular plates }(3,65)
$$

A. Subjected to a uniformly distributed lateral load

$$
w=\frac{q}{64 D}\left(a^{2}-r^{2}\right)^{2}
$$

B. Subjected to a concentrated load at the center

$$
w=\frac{p}{16 \pi D}\left[\left(a^{2}-r^{2}\right)+2 r^{2} \operatorname{Ln} \frac{r}{a}\right]
$$

C. Subjected to a uniformly distributed line load at $r=b$

I. Outer protion solution $r>b$

$$
w=\frac{P}{8 \pi D}\left[\left(a^{2}-r^{2}\right) \frac{a^{2}+b^{2}}{2 a^{2}}+\left(b^{2}+r^{2}\right) \operatorname{Ln} \frac{r}{a}\right]
$$


II. Inner portion solution $r<b$ and $r=b$

$$
w=\frac{p}{8 \pi D}\left[\left(b^{2}+r^{2}\right) \ln \frac{b}{a}+\left(a^{2}-b^{2}\right) \frac{\left(a^{2}+r^{2}\right)}{2 a^{2}}\right]
$$

Where for the aforementioned expressions of simply supported and clamped circular plates the following symbols are used:

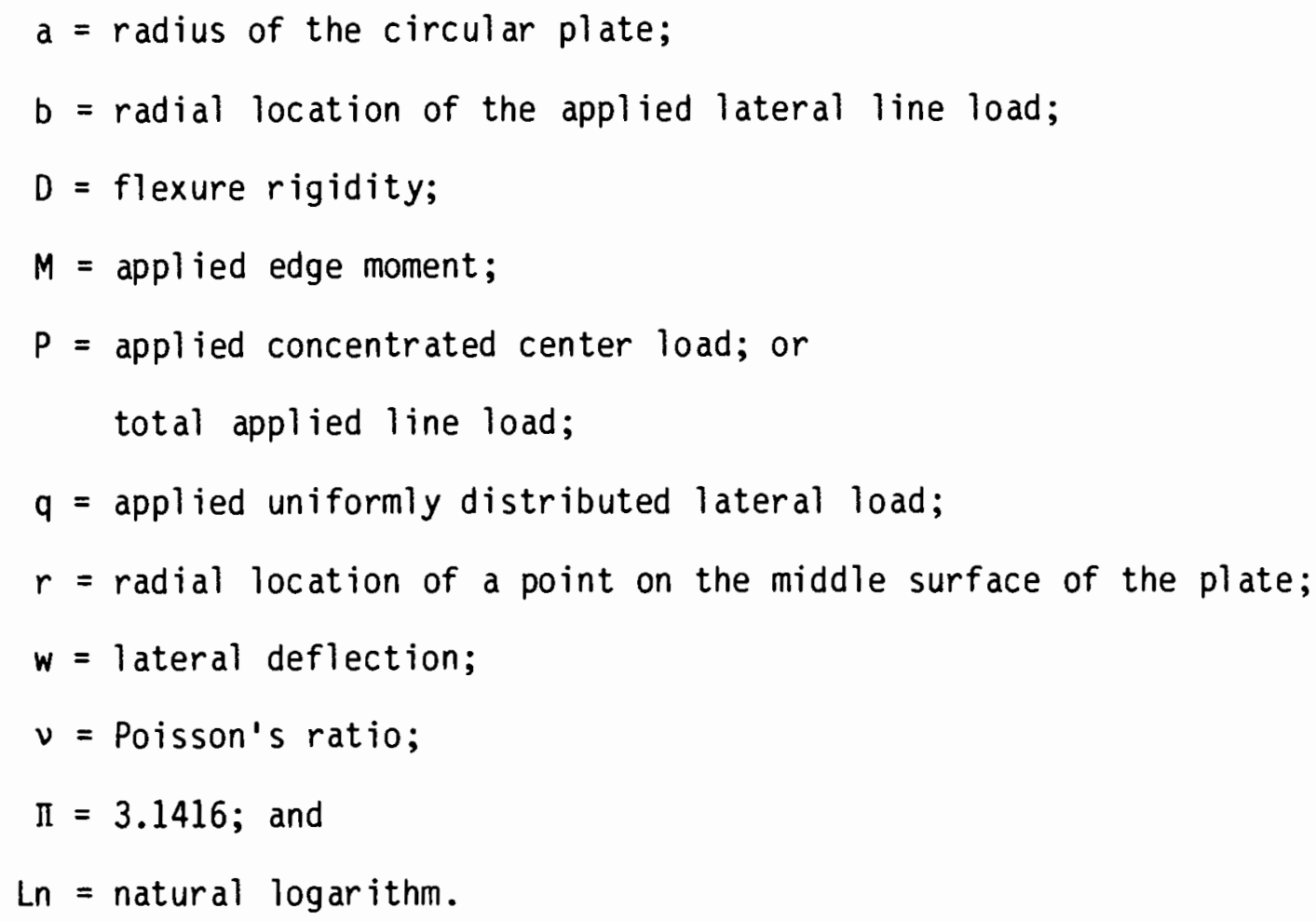




\section{Simply supported annular plates}

A. Subjected to a uniformly distributed lateral load

The solution of this problem may be obtained by superposing on the deflections obtained for a simply supported circular plate, equation (B.1), the deflections produced by the removal of the bending moments and the shearing forces of the simply supported circular plate at the inner edge location of the annular plate as shown in Fig. 51.a. These shearing forces and bending moments are given by (3)

$p=-q \pi b^{2}$

$M_{1}=-\frac{q}{16}(3+v)\left(a^{2}-b^{2}\right)$

Analytical expressions for the deflections produced by the inner edge bending moments and shearing forces are given by equations (B.15) and (B.14), respectively. Therefore, the solution of a simply supported annular plate subjected to a uniformly distributed lateral load may be expressed as

$$
\begin{gathered}
w=\frac{q}{64 D}\left(a^{2}-r^{2}\right)\left(\frac{5+v}{1+v} a^{2}-r^{2}\right)-\frac{q b^{2}}{4 D}\left[\frac{r^{2}}{2}\left(\ln \frac{r}{a}-1\right)-\frac{C_{1}}{4} r^{2}-C_{2} \operatorname{Ln} \frac{r}{a}+C_{3}\right] \\
-\frac{q b^{2}(3+v)}{16 D}\left[\frac{r^{2}}{2(1+v)}+\frac{a^{2}}{1-v} \ln \frac{r}{a}-\frac{a^{2}}{2(1+v)}\right]
\end{gathered}
$$



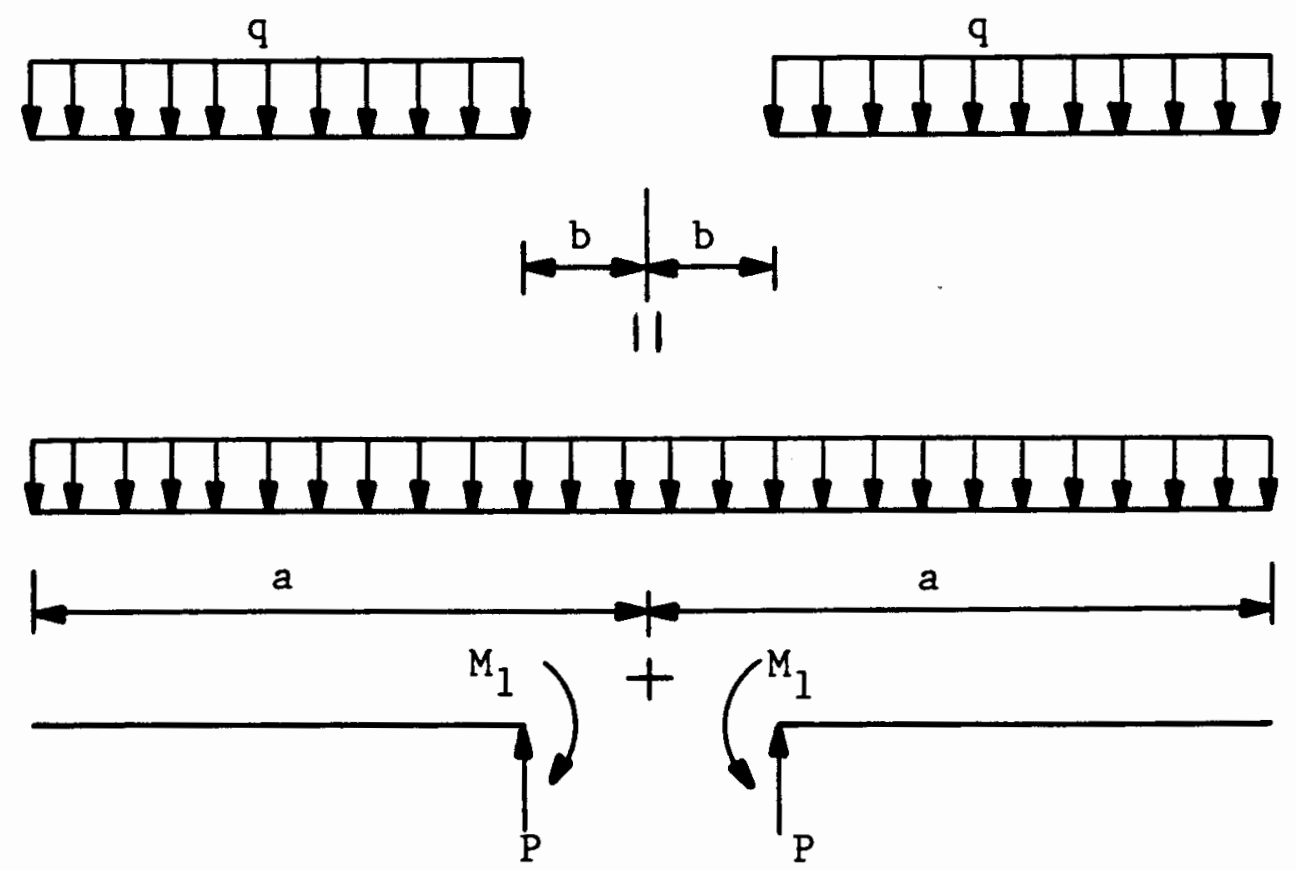

(a) SUBJECTED TO A UNIFORMLY DISTRIBUTED LATERAL LOAD

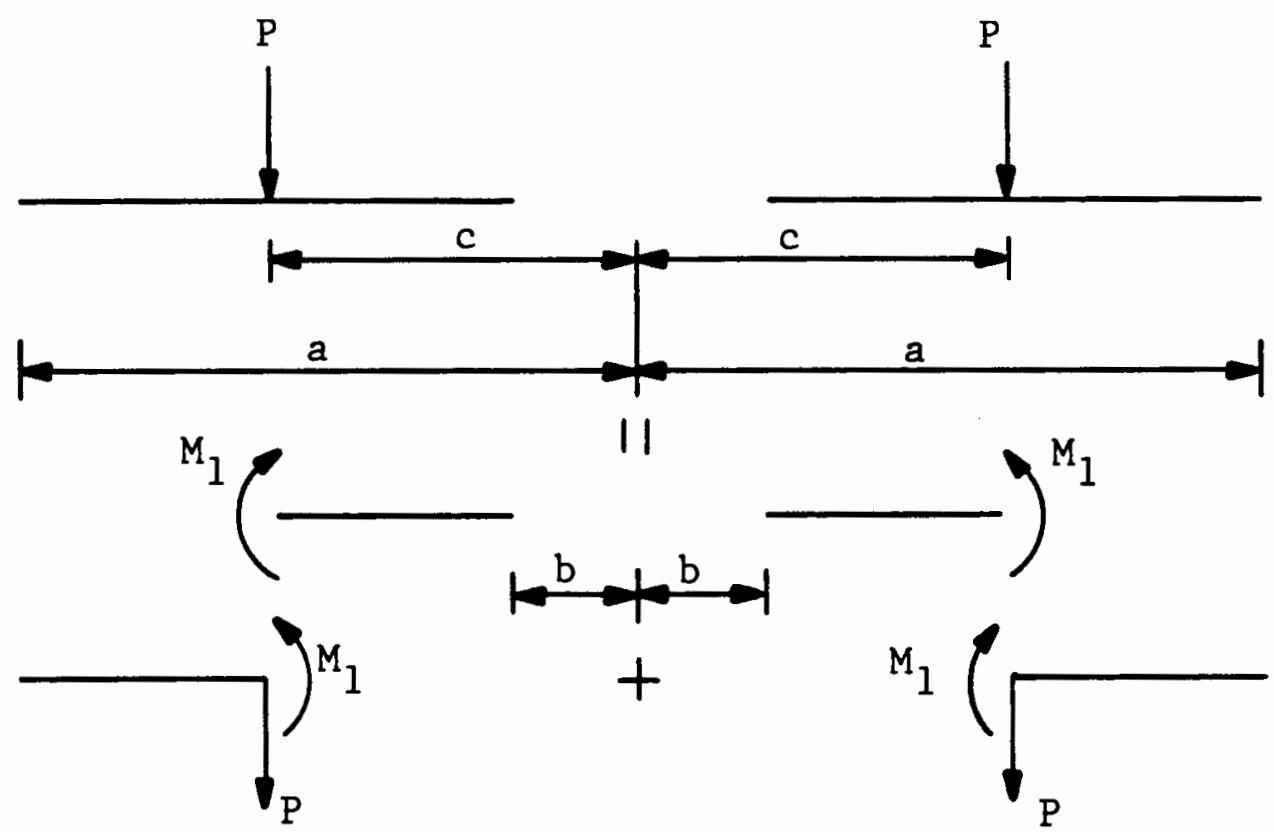

(b) SUBJECTED TO A UNIFORMLY DISTRIBUTED LATERAL LINE LOAD

FIGURE 51. DECOMPOSITION OF TWO LINEAR ANNULAR PLATE BEDNING PROBLEMS 
where

$$
\begin{aligned}
& c_{1}=\frac{1-v}{1+v}-\frac{2 b^{2}}{a^{2}-b^{2}} \ln \frac{b}{a} \\
& c_{2}=-\frac{1+v}{1-v} \frac{a^{2} b^{2}}{a^{2}-b^{2}} \ln \frac{b}{a} \\
& c_{3}=\frac{a^{2}}{2}\left(1+\frac{1}{2} \frac{1-v}{1+v}-\frac{b^{2}}{a^{2}-b^{2}} \ln \frac{b}{a}\right)
\end{aligned}
$$

B. Subjected to a uniformly distributed line load at the inner edge of the plate $(3,65)$

$$
w=\frac{P}{4 \Pi D}\left[\frac{r^{2}}{2}\left(\operatorname{Ln} \frac{r}{a}-1\right)-\frac{c_{1}}{4} r^{2}-c_{2} \operatorname{Ln} \frac{r}{a}+c_{3}\right]
$$

where $C_{1}, C_{2}$, and $C_{3}$ are defined by expressions (B.13).

C. Subjected to a uniformly distributed moments at the inner and outer edges $(3,65)$

$$
\begin{aligned}
& w=\frac{M_{1} b^{2}}{D\left(a^{2}-b^{2}\right)}\left[\frac{r^{2}}{2(1+v)}+\frac{a^{2}}{1-v} \operatorname{Ln} \frac{r}{a}-\frac{a^{2}}{2(1+v)}\right] \\
& \quad+\frac{M_{2} a^{2}}{D\left(a^{2}-b^{2}\right)}\left[-\frac{r^{2}}{2(1+v)}-\frac{b^{2}}{1-v} \ln \frac{r}{a}+\frac{a^{2}}{2(1+v)}\right]
\end{aligned}
$$

where $M_{1}$ and $M_{2}$ denote the uniformly distributed moments at the inner and outer edges, respectively. 
D. Subjected to a uniformly distributed line load at $r=c$ The solution of this problem may be obtained by dividing the simply supported annular plate into two parts as shown in Fig. 51.b. It is seen that the inner portion of the plate is in the condition of pure bending produced by the uniformly distributed moments $M_{1}$ and that the outer portion of the plate is bent by the moments $M_{1}$ and the shearing force $P$. The magnitude of the inner moment $M_{1}$ is found from the condition of continuity along the circle $r=c$, from which it follows that both portions of the plate have, at that circle, the same slope.

Since the shearing forces on the inner plate portion are equivalent to zero, the governing equation for the inner plate portion may, therefore, be expressed as $(3,65)$

$$
\frac{d}{d r}\left[\frac{1}{r} \frac{d}{d r}\left(r \frac{d w}{d r}\right)\right]=0
$$

Integrating the above expression with respect to $r$, we can write

$$
\begin{aligned}
& w=-\frac{I_{1}}{4} r^{2}-I_{2} \operatorname{Ln} r-I_{3} \\
& w_{r}=-\frac{I_{1}}{2} r-\frac{I_{2}}{r} \\
& w_{r r}=-\frac{I_{1}}{2}+\frac{I_{2}}{r^{2}}
\end{aligned}
$$


where $I_{1}, I_{2}$, and $I_{3}$ are constants of integration.

The sectional moment $M_{r}$ is given by

$M_{r}=-D\left(w,{ }_{r r}+\frac{v}{r} w,{ }_{r}\right)=D\left[\frac{1}{2}(1+v)-\frac{I_{2}}{r^{2}}(1-v)\right]$

At $r=b$, corresponding to the inner free edge, the sectional moment $M_{r}$ is equivalent to zero. At $r=c$, corresponding to the outer boundary of the inner plate portion, the sectional moment $M_{r}$ is equivalent to $M_{1}$. Substituting these boundary conditions into expressions (B.18) and solving the two resulting equations simultaneously, the values of the first and second constants of integration are found to equal

$$
\begin{aligned}
& I_{1}=\frac{2 M_{1}}{D(1+v)\left(1-\beta^{2}\right)} \\
& I_{2}=\frac{M_{1} b^{2}}{D(1-v)\left(1-\beta^{2}\right)}
\end{aligned}
$$

where

$$
\beta=\frac{b}{c}
$$

Substituting the values obtained for $I_{1}$ and $I_{2}$ into expression (B.17.b), the slope of the outer boundary of the inner portion of the plate $(r=c)$ may be expressed as 


$$
\left(w, r_{C}\right)_{C}=-\frac{M_{1} c}{D\left(1-\beta^{2}\right)}\left(\frac{1}{1+\nu}+\frac{\beta^{2}}{1-\nu}\right)
$$

The solution of the outer portion of the plate is readily obtained by superposing the deflections induced by the inner moment $M_{1}$, equation (B.15), on the deflections induced by the total shearing force $P$ given by equation (B.14). Thus, we can write

$$
\begin{aligned}
w= & \frac{M_{1} c^{2}}{D\left(a^{2}-c^{2}\right)}\left[\frac{r^{2}}{2(1+v)}+\frac{a^{2}}{1-v} \ln \frac{r}{a}-\frac{a^{2}}{2(1+v)}\right] \\
& +\frac{P}{4 \pi D}\left[\frac{r^{2}}{2}\left(\operatorname{Ln} \frac{r}{a}-1\right)-\frac{I_{4}}{4} r^{2}-I_{5} \ln \frac{r}{a}+I_{6}\right]
\end{aligned}
$$

where

$$
\begin{aligned}
& I_{4}=\frac{1-v}{1+v}-\frac{2 c^{2}}{a^{2}-c^{2}} \ln \frac{c}{a} \\
& I_{5}=-\frac{1+v}{1-v} \frac{a^{2} c^{2}}{a^{2}-c^{2}} \ln \frac{c}{a} \\
& I_{6}=\frac{a^{2}}{2}\left(1+\frac{1}{2} \frac{1-v}{1+v}-\frac{c^{2}}{a^{2}-c^{2}} \ln \frac{c}{a}\right)
\end{aligned}
$$

The slope of the inner boundary of the outer portion of the plate may, therefore, be expressed as 


$$
\begin{aligned}
\left(w, r^{\prime} c\right. & =\frac{M_{1} c^{2}}{D\left(a^{2}-c^{2}\right)}\left[\frac{c}{1+v}+\frac{a^{2}}{(1-v) c}\right] \\
& +\frac{P c}{4 \pi D}\left[\operatorname{Ln} \frac{c}{a}-\frac{1}{2}-\frac{I_{4}}{2}-\frac{I_{5}}{c^{2}}\right]
\end{aligned}
$$

Equating expressions (B.21) and (B.24) yields the value of the inner moments $M_{1}$ as a function of the total applied line load $P$

$$
M_{1}=-\frac{P c}{4 \Pi D} \frac{\operatorname{Ln} \frac{c}{a}-\frac{1}{2}-\frac{I_{4}}{2}-\frac{I_{5}}{c^{2}}}{\frac{c}{D\left(1-\beta^{2}\right)}\left(\frac{1}{1+v}+\frac{\beta^{2}}{1-v}\right)+\frac{c^{2}}{D\left(a^{2}-c^{2}\right)}\left[\frac{c}{1+v}+\frac{a^{2}}{(1-v) c}\right]}(B .25)
$$

I. Outer portion solution $r>c$ and $r=c$

The solution for this portion of the plate is given by expression (B.22) where $I_{4}, I_{5}, I_{6}$ and $M_{1}$ are given by equations (B.23) and (B.25), respectively.

II. Inner portion solution $b<r<c$ The deflection of the inner portion have been defined by equation (B.17.a)

$$
w=-\frac{I_{1}}{4} r^{2}-I_{2} \ln r-I_{3}
$$

where $I_{1}$ and $I_{2}$ are defined by equations (B.19). The value 
of $I_{3}$ is found from the condition of continuity along the circle $r=c$, from which it follows that both portions of the plate have, at that circle, the same deflection. Using equation (B.26), the third constant of integration is found to equal

$$
I_{3}=-\frac{I_{1}}{4} c^{2}-I_{2} \ln c-w_{c}
$$

Utilizing the continuity condition, the deflection at the outer boundary of the inner plate may be obtained from expression (B.22) as

$$
\begin{aligned}
w_{c}= & \frac{M_{1} c^{2}}{D\left(a^{2}-c^{2}\right)}\left[\frac{c^{2}}{2(1+v)}+\frac{a^{2}}{(1-v)} \ln \frac{c}{a}-\frac{a^{2}}{2(1+v)}\right] \\
& +\frac{P}{4 \pi D}\left[\frac{c^{2}}{2}\left(\operatorname{Ln} \frac{c}{a}-1\right)-\frac{I_{4}}{4} c^{2}-I_{5} \operatorname{Ln} \frac{c}{a}+I_{6}\right]
\end{aligned}
$$

Substituting expression (B.27) into (B.26), the solution corresponding to the inner portion is found to equal

$$
w=\frac{I_{1}}{4}\left(c^{2}-r^{2}\right)+I_{2} \ln \frac{c}{r}+w_{c}
$$

where $I_{1}, I_{2}$ and $w_{C}$ are given by expressions (B.19) and (B.28) respectively. 
Clamped annular plates

A. Subjected to a uniformily distributed lateral load

The solutionn of this problem may be obtained by superposing on the deflections obtained for a clamped circular plate, equation (B.6), the deflections produced by the removal of the bending moments and the shearing forces of the clamped circular plate at the inner edge location of the annular plate as shown in Fig. 51a. These shearing forces and bending moments are given by (53)

$p=-q \pi b^{2}$

$M_{1}=-\frac{q a^{2}}{16}\left[(1+\nu)-\frac{b^{2}}{a^{2}}(3+\nu)\right]$

Analytical expressions for deflections produced by the inner edge bending moments and shearing forces are given by expressions (B.35) and (B.34), respectively. Therefore, the solution of a clamped annular plate subjected to a uniformly distributed lateral load may be expressed as

$$
\begin{aligned}
& w=\frac{q}{640}\left(a^{2}-r^{2}\right)^{2}-\frac{q b^{2}}{8 D}\left[r^{2}\left(\operatorname{Ln} \frac{r}{a}-1\right)+\frac{1}{c_{4}}\left(-\frac{c_{1}}{4} r^{2}-C_{2} \operatorname{Ln} \frac{r}{a}+c_{3}\right)\right] \\
& -\frac{q a^{2}}{160 C_{4}}\left[(1+\nu)-\frac{b^{2}}{a^{2}}(3+\nu)\right]\left(-\frac{1}{2} r^{2}+a^{2} \operatorname{Ln} \frac{r}{a}+\frac{a^{2}}{2}\right)
\end{aligned}
$$


where

$$
\begin{aligned}
& c_{1}=2\left[(1+v)\left(2 \ln \frac{b}{a}\right)+(1-v)-\frac{a^{2}}{b^{2}}(1-v)\right] \\
& c_{2}=-a^{2}\left[(1+v)\left(2 \ln \frac{b}{a}\right)+2\right] \\
& c_{3}=\frac{a^{2}}{2}\left[(1+v)\left(2 \ln \frac{b}{a}\right)+(3+v)+\frac{a^{2}}{b^{2}}(1-v)\right] \\
& c_{4}=(1+v)+\frac{a^{2}}{b^{2}}(1-v)
\end{aligned}
$$

B. Subjected to a uniformly distributed line load at the inner edge of the plate (65)

$$
w=\frac{P}{8 \pi D}\left[r^{2}\left(\operatorname{Ln} \frac{r}{a}-1\right)+\frac{1}{C_{4}}\left(-\frac{C_{1}}{4} r^{2}-C_{2} \operatorname{Ln} \frac{r}{a}+C_{3}\right)\right]
$$

where $C_{1}, C_{2}, C_{3}$ and $C_{4}$ are defined by expressions ( $B .33$ )

C. Subjected to a uniformly distributed moment at the inner edge (65)

$$
w=\frac{M_{1}}{D C_{4}}\left(-\frac{1}{2} r^{2}+a^{2} \operatorname{Ln} \frac{r}{a}+\frac{a^{2}}{2}\right)
$$

where $C_{4}$ is defined by expression (B.33.d)

D. Subjected to a uniformly distributed line load at $r=c$ The solution of this problem may be obtained by dividing the 
clamped annular plate into two parts as shown in Fig. 51.b. Following a procedure similar to that outlined for simply supported annular plates in section 3D, we obtain

$$
M_{1}=-\frac{P c}{8 \pi D} \frac{\left(2 \operatorname{Ln} \frac{c}{a}-1\right)+\frac{1}{I_{4}}\left(-\frac{I_{5}}{2}-\frac{I_{6}}{c^{2}}\right)}{\frac{c}{D\left(1-\beta^{2}\right)}\left(\frac{1}{1+v}+\frac{\beta^{2}}{1-v}\right)+\frac{1}{D I_{4}}\left(-c+\frac{a^{2}}{c}\right)}
$$

where

$$
\begin{aligned}
& I_{4}=(1+\nu)+\frac{a^{2}}{c^{2}}(1-\nu) \\
& I_{5}=2\left[(1+\nu)\left(2 \ln \frac{c}{a}\right)+(1-\nu)\left(1-\frac{a^{2}}{c^{2}}\right)\right] \\
& I_{6}=-a^{2}\left[(1+\nu)\left(2 \ln \frac{c}{a}\right)+2\right] \\
& \beta=\frac{b}{c}
\end{aligned}
$$

I. Outer portion $r>c$ and $r=c$ Substituting the expression for $M_{1}(B .36)$ into equation (B.35), we obtain the deflections due to moments $M_{1}$. The deflections due to the total shearing force $P$ are obtained from equation (B.34). Adding together both these deflections we 
obtain the outer portion solution stated as

$$
\begin{aligned}
w= & \frac{M_{1}}{D_{4}}\left(-\frac{1}{2} r^{2}+a^{2} \operatorname{Ln} \frac{r}{a}+\frac{a^{2}}{2}\right) \\
& +\frac{P}{8 \pi}\left[r^{2}\left(\operatorname{Ln} \frac{r}{a}-1\right)+\frac{1}{I_{4}}\left(-\frac{I_{5}}{4} r^{2}-I_{6} \operatorname{Ln} \frac{r}{a}+I_{7}\right)\right]
\end{aligned}
$$

where

$$
I_{7}=\frac{a^{2}}{2}\left[(1+v)\left(2 \ln \frac{c}{a}\right)+(3+v)+\frac{a^{2}}{c^{2}}(1-v)\right]
$$

and $I_{4}, I_{5}, I_{6}$ and $M_{1}$ are given by equations (B.37) and (B.36), respectively.

II. Inner portion solution $b<r<c$

The inner portion solution for fixed annular plates is equivalent to that of simply supported annular plates outlined in section 3.D.II. Thus, we can write

$$
w=\frac{I_{1}}{4}\left(c^{2}-r^{2}\right)+I_{2} \ln \frac{c}{r}+w_{c}
$$

where $I_{1}$ and $I_{2}$ are given by equation (B.19) Utilizing the continuity condition, the deflection at the outer boundary of the inner plate portion may be obtained from equation (B.39) as 


$$
\begin{aligned}
w_{c} & =\frac{M_{1}}{D I_{4}}\left(-\frac{1}{2} c^{2}+a^{2} \operatorname{Ln} \frac{c}{a}+\frac{a}{2}\right) \\
& +\frac{P}{8 \Pi D}\left[c^{2}\left(\operatorname{Ln} \frac{c}{a}-1\right)+\frac{1}{I_{4}}\left(-\frac{I_{5}}{4} c^{2}-I_{6} \operatorname{Ln} \frac{c}{a}+I_{7}\right)\right]
\end{aligned}
$$

where $M_{1}$ and $I_{4}, I_{5}, I_{6}, I_{7}$ are defined by equations (B.36), and (B.40), respectively.

For the aforementioned simply supported and fixed annular plate expressions the following symbols are used:

$$
\begin{aligned}
a & =\text { radius of plate; } \\
b & =\text { radius of the circular hole at the center of the plate; } \\
c & =\text { radial location of applied line load; } \\
D & =\text { elastic flexural rigidity; } \\
M_{1}, M_{2} & =\text { applied inner edge and outer edge moments; } \\
M_{r} & =\text { sectional moment per unit length; } \\
P & =\text { total applied line load; } \\
q & =\text { applied uniformly distributed lateral load; } \\
r & =\text { radial location of a point on the middle surface of the } \\
& \text { plate; } \\
w & =\text { lateral deflection; } \\
\nu & =\text { Poisson's ratio; } \\
\Pi & =3.1416 ; \text { and } \\
L n & =\text { natural logarithim. }
\end{aligned}
$$

